\title{
The Total Synthesis of Plakortone B and Analogs
}

\author{
Martin F. Semmelhack ${ }^{\star}$, Richard J. Hooley and Christina Kraml \\ Department of Chemistry, Frick Chemistry Laboratory, Princeton University, Princeton, New Jersey 08544 \\ mfshack@princeton.edu
}

\section{Supporting Information}

\section{General Information}

Analytical Data: NMR spectra were recorded with a Varian Unity/Mercury $300(300 \mathrm{MHz}$ for $\left.{ }^{1} \mathrm{H}\right)$, a Varian Unity/Inova $400\left(400 \mathrm{MHz}\right.$ for $\left.{ }^{1} \mathrm{H}\right)$ or a Varian/Unity Inova $500\left(500 \mathrm{MHz}\right.$ for $\left.{ }^{1} \mathrm{H}\right)$ instrument. Proton $\left({ }^{1} \mathrm{H}\right)$ chemical shifts, reported in parts per million (ppm) were indirectly referenced to external tetramethylsilane employing resonances due to trace monoprotio-solvent as an internal reference. Multiplicities are abbreviated: singlet, s; doublet, d; triplet, t; quartet, q; quintet, qn; sextet, sx; septet, sp; multiplet, m. Carbon-13 $\left({ }^{13} \mathrm{C}\right)$ chemical shifts reported in parts per million $(\mathrm{ppm})$ were indirectly referenced to external tetramethylsilane employing known solvent resonances as an internal reference. Infrared (IR) spectra were recorded on a Nicolet 800 FT-IR spectrometer with internal calibration. Mass spectral data were obtained using a Kratos MS 80 spectrometer operating in either electron impact mode (EI) at $70 \mathrm{eV}$, fast atom bombardment mode (FAB), or electrospray mode (ES). Peaks are identified by mass/charge ratio $(\mathrm{m} / \mathrm{z})$ and relative abundance to a base peak $(\%)$.

Solvents: Anhydrous diethyl ether (ether) and tetrahydrofuran (THF) were obtained by distillation from sodium benzophenone ketyl. Benzene, toluene, dichloromethane, hexanes, N,Ndimethylformamide (DMF), hexamethylphosphoramide (HMPA) and acetonitrile were distilled from $\mathrm{CaH}_{2}$ prior to use. Methanol was distilled from $\mathrm{CaSO}_{4}$ prior to use. Triethylamine was 
purified by passing through a solvent purification column prior to use. Other solvents were purchased from Aldrich Chemical Company in anhydrous form under Sure-Seal ${ }^{\circledR}$ packing and used without further purification.

Chromatography: Flash Column Chromatography was performed with Sorbent Technologies Standard Grade Silica Gel (60 A porosity, 230 x 450 mesh) with the indicated solvents. The gel was "wet-packed" by loading into a glass column as a slurry in hexanes and run with air pressure. Analytical thin layer chromatography was conducted on Sorbent Technologies UV254 polyester backed silica plates $(0.20 \mathrm{~mm})$ with fluorescent indicator. Developed plates were visualized under UV light or by dipping in a solution of either 5\% ethanolic $p$-anisaldehyde solution, a $5 \%$ ethanolic solution of phosphomolybdic acid or aqueous potassium permanganate followed by heating.

Reagents: Commercial reagents were purchased from Aldrich Chemical Company, Acros Organics, Fluka or Strem and used without further purification, unless otherwise noted. Lithium diisopropylamide was prepared from diisopropylamine in THF cooled to $-78{ }^{\circ} \mathrm{C}$ to which was added dropwise 0.98 mol-eq of $n$-BuLi. The reaction was warmed to $-10{ }^{\circ} \mathrm{C}$ for $1 \mathrm{~h}$ then cooled to $-78^{\circ} \mathrm{C}$ prior to use. Lithium hexamethyldisilazide was prepared from hexamethyldisilazane in THF cooled to $-78{ }^{\circ} \mathrm{C}$ to which was added dropwise 0.98 mol-eq of $n$-BuLi. The reaction was warmed to $-10^{\circ} \mathrm{C}$ for $1 \mathrm{~h}$ then cooled to $-78^{\circ} \mathrm{C}$ prior to use.

General Procedures: Reactions "under argon" atmosphere were performed in glassware dried in an oven at $160{ }^{\circ} \mathrm{C}$ for several hours, then cooled to room temperature in a desiccator over $\mathrm{CaSO}_{4}$. The glassware, connected to a double manifold, was sequentially evacuated (0.05 $\mathrm{mmHg}$ ) and filled with argon three times before use. Low boiling solvents were removed on a Büchi Rotovapor-R under vacuum (15-20 $\mathrm{mmHg}$ ) provided by a water aspirator pump. NMR tubes were dried in a horizontal position over several hours at $140{ }^{\circ} \mathrm{C}$ and stored at $70{ }^{\circ} \mathrm{C}$. Syringes and needles were dried at $140{ }^{\circ} \mathrm{C}$ and stored at $70{ }^{\circ} \mathrm{C}$. Solid residues and oils obtained by evaporation of solvents were routinely dried on a vacuum line $(0.05 \mathrm{mmHg})$ at $23{ }^{\circ} \mathrm{C}$. 


\section{Synthesis and Characterization of New Molecules:}

\section{Synthesis of Plakortone Core:}

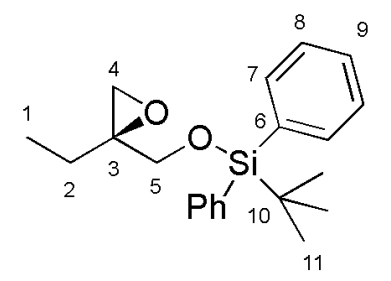

6

Preparation of $(\boldsymbol{R})$-tert-Butyl-(2-ethyloxiranylmethoxy)diphenylsilane 6: To a 25-mL, threenecked, round-bottomed flask equipped with a magnetic stirring bar was added imidazole (170 $\mathrm{mg}, 2.50 \mathrm{mmol}$ ). The flask was placed under an argon atmosphere, and dimethylformamide (5 $\mathrm{mL}$ ) was added via syringe. The mixture was cooled to $0{ }^{\circ} \mathrm{C}$ and $(R)$-(2-ethyloxiranyl)methanol ${ }^{1}$ $102 \mathrm{mg}, 1.00 \mathrm{mmol})$ in DMF $(1 \mathrm{~mL})$ was added via syringe. Tert-butyldiphenylchlorosilane $(1.30 \mathrm{mmol}, 357 \mathrm{mg})$ was added dropwise via syringe, and the mixture stirred at $23{ }^{\circ} \mathrm{C}$ for $15 \mathrm{~h}$. Ether $(50 \mathrm{~mL})$ was added, followed by saturated aqueous $\mathrm{NH}_{4} \mathrm{Cl}$. The mixture was transferred to a separatory funnel and the layers separated. The organic layer was washed with water (4 x 10 $\mathrm{mL})$ and saturated brine $(10 \mathrm{~mL})$, dried $\left(\mathrm{MgSO}_{4}\right)$, filtered and the solvent removed by rotary evaporation. The resulting yellow oil was subjected to column chromatography $\left(\mathrm{SiO}_{2}\right.$; hexanes/ether 9:1) to yield 6 (289 mg, 85\%) as a colorless oil, $\mathrm{R}_{\mathrm{f}} 0.5$ (hexanes/ether $\left.4: 1\right) ;{ }^{1} \mathrm{H}$ NMR $\left(400 \mathrm{MHz}, \mathrm{CDCl}_{3}\right) \delta 0.89\left(\mathrm{t}, \mathrm{J}=7.5 \mathrm{~Hz}, \mathrm{H}_{1}\right) ; 1.04\left(\mathrm{~s}, 9 \mathrm{H}, \mathrm{H}_{11}\right) ; 1.65$ (dq, J = 15.0, $7.5 \mathrm{~Hz}$, $\left.1 \mathrm{H}, \mathrm{H}_{2}\right) ; 1.80\left(\mathrm{dq}, \mathrm{J}=15.0,7.5 \mathrm{~Hz}, 1 \mathrm{H}, \mathrm{H}_{2}\right) ; 2.59\left(\mathrm{~d}, \mathrm{~J}=5.0 \mathrm{~Hz}, 1 \mathrm{H}, \mathrm{H}_{5}\right) ; 2.64(\mathrm{~d}, \mathrm{~J}=5.0 \mathrm{~Hz}, 1 \mathrm{H}$, $\left.\mathrm{H}_{5}\right) ; 3.68\left(\mathrm{~s}, 2 \mathrm{H}, \mathrm{H}_{4}\right) ; 7.34-7.44\left(\mathrm{~m}, 6 \mathrm{H}, \mathrm{H}_{8}, \mathrm{H}_{9}\right) ; 7.63-7.68\left(\mathrm{~m}, 4 \mathrm{H}, \mathrm{H}_{7}\right) ;{ }^{13} \mathrm{C} \mathrm{NMR}(125 \mathrm{MHz}$, $\left.\mathrm{CDCl}_{3}\right)$ \& $8.9\left(\mathrm{C}_{1}\right) ; 19.5\left(\mathrm{C}_{10}\right) ; 24.7\left(\mathrm{C}_{2}\right) ; 27.0\left(\mathrm{C}_{11}\right) ; 50.2\left(\mathrm{C}_{5}\right) ; 60.6\left(\mathrm{C}_{3}\right) ; 65.7\left(\mathrm{C}_{4}\right) ; 127.9\left(\mathrm{C}_{8}\right)$; $129.9\left(\mathrm{C}_{9}\right) ; 133.5\left(\mathrm{C}_{6}\right) ; 135.8\left(\mathrm{C}_{7}\right)$; IR ( $\mathrm{NaCl}$, thin film) $\tilde{v}_{\max }\left(\mathrm{cm}^{-1}\right) ; 740,823,1113,1390,1472$, 2857, 2931; EIMS m/z: 283 (M-C $\left.\mathrm{H}_{9}{ }^{+}, 52\right), 253$ (82), 205 (80), 199 (100), 186 (93), 175 (83), 123 (66), 105 (55), 91 (42), 77 (70). EIHRMS m/z: calcd for $\mathrm{C}_{17} \mathrm{H}_{19} \mathrm{O}_{2} \mathrm{Si}\left(\mathrm{M}_{-} \mathrm{C}_{4} \mathrm{H}_{9}{ }^{+}\right), 283.11543$; found 283.11592 . 


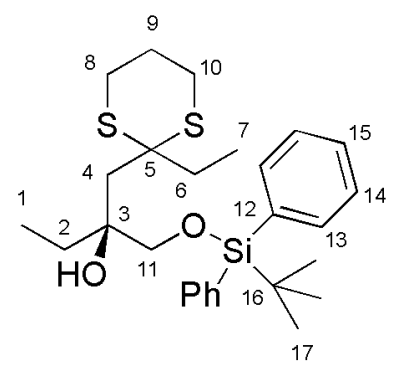

S-1

Preparation of (S)-2-(tert-Butyldiphenylsilanyloxymethyl)-1-(2-ethyl-1,3-dithian-2-

yl)butan-2-ol S-1: A 1-L, oven-dried, three-necked, round-bottomed flask was equipped with a magnetic stirring bar and placed under an argon atmosphere. THF $(500 \mathrm{~mL})$ and 2-ethyl-1,3dithiane (7.92 g, $53.5 \mathrm{mmol}$, in $20 \mathrm{~mL}$ THF) were added via syringe and cooled to $-30{ }^{\circ} \mathrm{C}$ with a constant temperature bath. $n$-BuLi $(53.5 \mathrm{mmol}, 1.6 \mathrm{M}$ in hexanes, $33.5 \mathrm{~mL})$ was added via syringe over $10 \mathrm{~min}$. The solution was stirred for $1 \mathrm{~h}$, and $\mathbf{6}$ (14.0 g, $41.2 \mathrm{mmol}$, in $50 \mathrm{~mL}$ THF) was added dropwise via syringe. The reaction was stirred at $-30{ }^{\circ} \mathrm{C}$ for $15 \mathrm{~h}$. Saturated $\mathrm{NH}_{4} \mathrm{Cl}$ $(100 \mathrm{~mL})$ was added, and the reaction warmed to $23{ }^{\circ} \mathrm{C}$. The mixture was transferred to a separatory funnel and diluted with ether $(2.5 \mathrm{~L})$. The layers were separated, and the aqueous layer extracted with ether $(3 \times 50 \mathrm{~mL})$. The combined organic layers were washed with saturated brine $(100 \mathrm{~mL})$ and water $(100 \mathrm{~mL})$, dried over $\mathrm{MgSO}_{4}$, filtered and the solvent removed by rotary evaporation. The resulting crude yellow oil was purified by column chromatography ( $\mathrm{SiO}_{2}$ : hexanes/ether 19:1) to yield $\mathbf{S - 1}(19.5 \mathrm{~g}, 97 \%)$ as a pale yellow oil, $\mathrm{R}_{\mathrm{f}} 0.3$ (hexanes/ether 9:1); ${ }^{1} \mathrm{H}$ NMR (400 MHz, $\left.\mathrm{CDCl}_{3}\right) \delta 0.84\left(\mathrm{t}, \mathrm{J}=7.5 \mathrm{~Hz}, 3 \mathrm{H}, \mathrm{H}_{7}\right) ; 1.04$ (t, J = 7.5 Hz, 3H, $\left.\mathrm{H}_{1}\right) ; 1.08$ (s, 9H, $\left.\mathrm{H}_{17}\right) ; 1.68\left(\mathrm{dq}, \mathrm{J}=15.0,7.5 \mathrm{~Hz}, 1 \mathrm{H}, \mathrm{H}_{6}\right) ; 1.73\left(\mathrm{dq}, \mathrm{J}=15.0,7.5 \mathrm{~Hz}, 1 \mathrm{H}, \mathrm{H}_{6}\right) ; 1.82-1.90$ (m, 2H, H9); 2.09 (q, J = 7.5 Hz, 2H, H $\left.\mathrm{H}_{2}\right) ; .10$ (d, J = $\left.15.0 \mathrm{~Hz}, 1 \mathrm{H}, \mathrm{H}_{4}\right) ; 2.27$ (d, J = $15.0 \mathrm{~Hz}, 1 \mathrm{H}$, $\left.\mathrm{H}_{4}\right) ; 2.67-2.84\left(\mathrm{~m}, 4 \mathrm{H}, \mathrm{H}_{8}, \mathrm{H}_{10}\right) ; 3.41(\mathrm{~s}, 1 \mathrm{H}, \mathrm{OH}) ; 3.53\left(\mathrm{~d}, \mathrm{~J}=11.0 \mathrm{~Hz}, 1 \mathrm{H}, \mathrm{H}_{11}\right) ; 3.62(\mathrm{~d}, \mathrm{~J}=$ 11.0 Hz, 1H, $\mathrm{H}_{11}$ ); 7.31-7.44 (m, 6H, $\left.\mathrm{H}_{14}+\mathrm{H}_{15}\right)$; 7.60-7.70 (m, 4H, $\left.\mathrm{H}_{13}\right) ;{ }^{13} \mathrm{C} \mathrm{NMR}(125 \mathrm{MHz}$, $\left.\mathrm{CDCl}_{3}\right)$ \& $8.0\left(\mathrm{C}_{7}\right) ; 9.5\left(\mathrm{C}_{1}\right) ; 19.5\left(\mathrm{C}_{16}\right) ; 25.1\left(\mathrm{C}_{2}\right) ; 26.6\left(\mathrm{C}_{8}\right) ; 26.7\left(\mathrm{C}_{10}\right) ; 27.2\left(\mathrm{C}_{17}\right) ; 31.9\left(\mathrm{C}_{6}\right)$; $33.3\left(\mathrm{C}_{9}\right)$; $41.6\left(\mathrm{C}_{4}\right) ; 53.7\left(\mathrm{C}_{5}\right) ; 68.9\left(\mathrm{C}_{3}\right) ; 75.8\left(\mathrm{C}_{11}\right) ; 127.9\left(\mathrm{C}_{14}\right) ; 129.9\left(\mathrm{C}_{15}\right) ; 133.5\left(\mathrm{C}_{12}\right) ; 136.0$ $\left(\mathrm{C}_{13}\right)$; IR $\left(\mathrm{NaCl}\right.$, thin film) $\tilde{v}_{\max }\left(\mathrm{cm}^{-1}\right) ; 613,702,740,824,908,999,1112,1275,1390,1427$, 1472, 1589, 2857, 2932, 2962, 3049, 3070, 3423: EIMS m/z: $269\left(\mathrm{M}^{+}, 2\right), 470$ (6), 431 (9), 395 (12), 323 (17), 271 (42), 270 (81), 269 (97) 249 (88), 215 (60), 199 (90), 135 (100), 107 (78), 88 (40), 77 (67); HRMS-EI $m / z$ : calcd for $\mathrm{C}_{27} \mathrm{H}_{40} \mathrm{O}_{2} \mathrm{~S}_{2} \mathrm{Si}$, 488.22424; found 488.22532. 


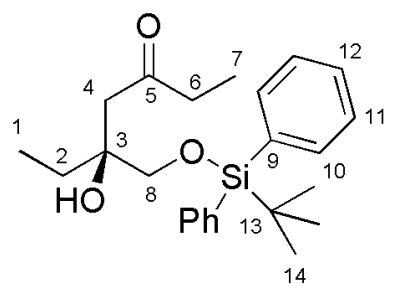

7

Preparation of (S)-5-(tert-Butyldiphenylsilanyloxymethyl)-5-hydroxyheptan-3-one 7: To a 500 -mL round-bottomed flask equipped with a magnetic stirring bar was added mercuric oxide (20.6 mmol, $4.46 \mathrm{~g}$ ), mercuric chloride $(27.5 \mathrm{mmol}, 4.46 \mathrm{~g}$ ) and a 9:1:1 mixture of methanol:isopropanol:water (300 mL). Dithiane S-1 (6.70 g, $13.73 \mathrm{mmol}$, in $20 \mathrm{~mL}$ isopropanol) was added and the reaction stirred for $24 \mathrm{~h}$. The reaction mixture was filtered through a Celite pad and the solvent removed by rotary evaporation. Hexanes/ether $(1: 1,300 \mathrm{~mL})$ was added, and the solution transferred to a separatory funnel. The aqueous layer was removed, and the organic layer washed with water $(4 \times 30 \mathrm{~mL})$, then dried $\left(\mathrm{MgSO}_{4}\right)$, filtered and the solvent removed by rotary evaporation. (NOTE: If any white solid is observed at this point, redissolve in hexanes/ether and repeat the washing procedure. It is important that no mercury salts are present before chromatographic purification). The resulting crude oil was purified by column chromatography $\left(\mathrm{SiO}_{2}\right.$ : hexanes/ether $\left.4: 1\right)$ to yield ketone $7(4.89 \mathrm{~g}, 79 \%)$ as a colorless oil, which crystallizes on freezing (mp $10{ }^{\circ} \mathrm{C}$ ), $\mathrm{R}_{\mathrm{f}} 0.25$ (hexanes/ether 4:1); ${ }^{1} \mathrm{H}$ NMR (400 MHz, $\left.\mathrm{CDCl}_{3}\right) \delta 0.82\left(\mathrm{t}, \mathrm{J}=7.5 \mathrm{~Hz}, 3 \mathrm{H}, \mathrm{H}_{7}\right) ; 0.98\left(\mathrm{t}, \mathrm{J}=7.5 \mathrm{~Hz}, 3 \mathrm{H}, \mathrm{H}_{1}\right) ; 1.04\left(\mathrm{~s}, 9 \mathrm{H}, \mathrm{H}_{14}\right) ; 1.55(\mathrm{q}, \mathrm{J}=$ $\left.7.5 \mathrm{~Hz}, 1 \mathrm{H}, \mathrm{H}_{2}\right) ; 1.56\left(\mathrm{q}, \mathrm{J}=7.5 \mathrm{~Hz}, 1 \mathrm{H}, \mathrm{H}_{2}\right) ; 2.42$ (q, J = 7.5 Hz, 2H, $\left.\mathrm{H}_{6}\right) ; 2.49$ (d, J = $16.0 \mathrm{~Hz}$, $\left.1 \mathrm{H}, \mathrm{H}_{4}\right) ; 2.76\left(\mathrm{~d}, \mathrm{~J}=16.0 \mathrm{~Hz}, 1 \mathrm{H}, \mathrm{H}_{4}\right) ; 3.51\left(\mathrm{~s}, 2 \mathrm{H}, \mathrm{H}_{8}\right) ; 3.82(\mathrm{~s}, 1 \mathrm{H}, \mathrm{OH}) ; 7.35-7.44(\mathrm{~m}, 6 \mathrm{H}$, $\left.\mathrm{H}_{11}+\mathrm{H}_{12}\right)$; 7.60-7.70 (m, 4H, $\left.\mathrm{H}_{13}\right) ;{ }^{13} \mathrm{C}$ NMR $\left(125 \mathrm{MHz}, \mathrm{CDCl}_{3}\right) \delta$ 7.6 $\left(\mathrm{C}_{1}\right) ; 7.6\left(\mathrm{C}_{7}\right) ; 19.5\left(\mathrm{C}_{13}\right)$; $27.1\left(\mathrm{C}_{14}\right) ; 30.1\left(\mathrm{C}_{2}\right)$ : $38.0\left(\mathrm{C}_{6}\right) ; 46.5\left(\mathrm{C}_{4}\right) ; 68.2\left(\mathrm{C}_{3}\right) ; 74.5\left(\mathrm{C}_{8}\right) ; 127.9\left(\mathrm{C}_{11}\right) ; 130.0\left(\mathrm{C}_{12}\right) ; 133.3$ $\left(\mathrm{C}_{9}\right) ; 135.8\left(\mathrm{C}_{10}\right) ; 215.1\left(\mathrm{C}_{5}\right)$; IR ( NaCl, thin film) $\tilde{v}_{\max }\left(\mathrm{cm}^{-1}\right)$; 702, 823, 1112, 1185, 1427, 1472,

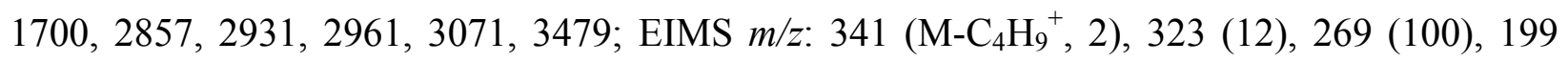
(89), 191 (100), 139 (71), 135 (53), 105 (31), 77, (39); ESMS m/z: $421\left(\mathrm{M}+\mathrm{Na}^{+}\right)$. 


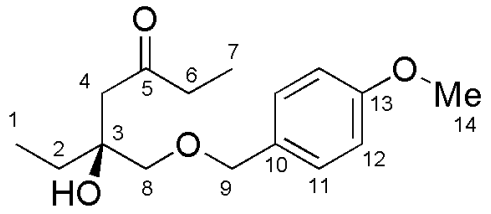

Characterization of (S)-5-Hydroxy-5-(4-methoxybenzyloxymethyl)heptan-3-one S-2: $p$ Methoxybenzyl ether S-2 was synthesized from known epoxide (R)-ethyl-2-(4methoxybenzyloxymethyl)oxirane by the same route used to synthesize $7, \mathrm{R}_{\mathrm{f}} 0.25$ (hexanes/ether 9:1); ${ }^{1} \mathrm{H}$ NMR (400 MHz, $\left.\mathrm{CDCl}_{3}\right) \delta 0.88\left(\mathrm{t}, \mathrm{J}=7.5 \mathrm{~Hz}, 3 \mathrm{H}, \mathrm{H}_{7}\right) ; 1.00\left(\mathrm{t}, \mathrm{J}=7.5 \mathrm{~Hz}, 3 \mathrm{H}, \mathrm{H}_{1}\right)$; $1.56\left(\mathrm{dq}, \mathrm{J}=7.5,13.0 \mathrm{~Hz}, 1 \mathrm{H}, \mathrm{H}_{2}\right) ; 1.57\left(\mathrm{dq}, \mathrm{J}=7.5,13.0 \mathrm{~Hz}, 1 \mathrm{H}, \mathrm{H}_{2}\right) ; 2.44$ (dq, J = 7.5, 13.0 $\left.\mathrm{Hz}, 1 \mathrm{H}, \mathrm{H}_{6}\right) ; 2.45$ (dq, J = 7.5, 13.0 Hz, 1H, H6); 2.49 (d, J = $\left.16.5 \mathrm{~Hz}, 1 \mathrm{H}, \mathrm{H}_{4}\right) ; 2.76$ (d, J = 16.5 $\left.\mathrm{Hz}, 1 \mathrm{H}, \mathrm{H}_{4}\right) ; 3.36\left(\mathrm{~s}, 2 \mathrm{H}, \mathrm{H}_{8}\right) ; 3.81\left(\mathrm{~s}, 3 \mathrm{H}, \mathrm{H}_{14}\right) ; 4.08(\mathrm{~s}, 1 \mathrm{H}, \mathrm{OH}) ; 4.44\left(\mathrm{~s}, 2 \mathrm{H}, \mathrm{H}_{9}\right) ; 6.88(\mathrm{~d}, \mathrm{~J}=$ $\left.8.5 \mathrm{~Hz}, 2 \mathrm{H}, \mathrm{H}_{12}\right) ; 7.23\left(\mathrm{~d}, \mathrm{~J}=8.5 \mathrm{~Hz}, 2 \mathrm{H}, \mathrm{H}_{11}\right) ;{ }^{13} \mathrm{C} \mathrm{NMR}\left(125 \mathrm{MHz}, \mathrm{CDCl}_{3}\right) \delta 7.6\left(\mathrm{C}_{1}\right) ; 7.8\left(\mathrm{C}_{7}\right)$; $30.8\left(\mathrm{C}_{2}\right): 38.1\left(\mathrm{C}_{6}\right) ; 46.6\left(\mathrm{C}_{4}\right) ; 55.5\left(\mathrm{C}_{14}\right) ; 73.4\left(\mathrm{C}_{3}\right) ; 74.3\left(\mathrm{C}_{8}\right) ; 74.9\left(\mathrm{C}_{9}\right) ; 114.0\left(\mathrm{C}_{12}\right) ; 129.7$ $\left(\mathrm{C}_{11}\right) ; 130.3\left(\mathrm{C}_{10}\right) ; 159.5\left(\mathrm{C}_{13}\right) ; 214.8\left(\mathrm{C}_{5}\right)$; IR $\left(\mathrm{NaCl}\right.$, thin film) $\tilde{v}_{\max }\left(\mathrm{cm}^{-1}\right) ; 827,1035,1093$, 1175, 1248, 1303, 1361, 1462, 1513, 1612, 1700, 2938, 2971, 3471; EIMS m/z: $204\left(\mathrm{M}^{+}, 1\right), 202$ (8), 137 (81), 135 (42), 122 (49), 121 (200), 105 (29), 91 (28), 77 (52). 
Measurement of enantiomeric excess: S-2 was analyzed by chiral HPLC, using a Chiralpak AS $(25 \times 0.46 \mathrm{~cm})$ column, eluted with 8:2 hexane:isopropanol at a rate of $1 \mathrm{ml} / \mathrm{min}$, UV detection at $254 \mathrm{~nm}$. Enantiomeric excess of $\mathbf{S}-\mathbf{2}=85 \%$.

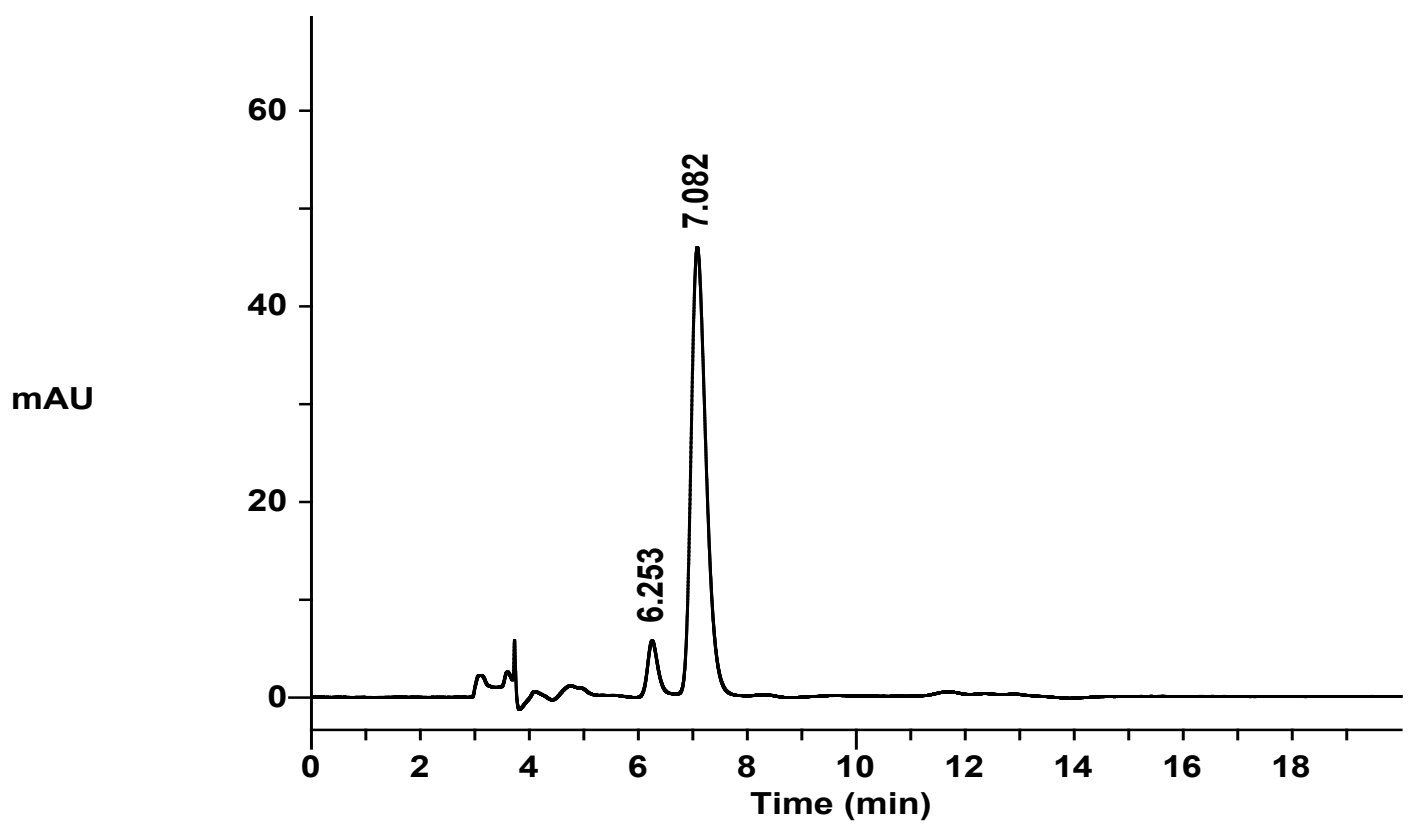

Scheme S-1 Measurement of enantiomeric excess of ketone S-2

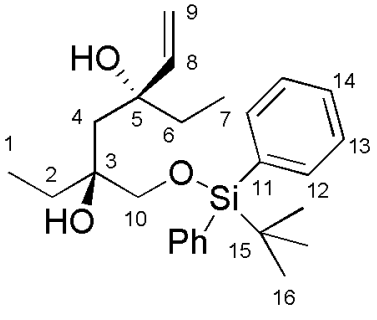

9

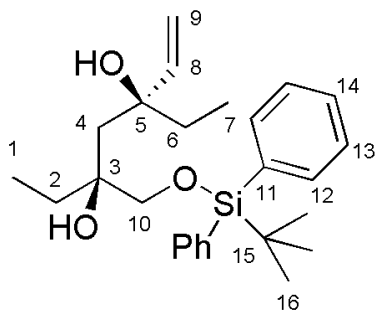

8

Preparation of (3R,5S)-5-(tert-Butyldiphenylsilanyloxymethyl)-3-ethylhept-1-ene-3,5-diol 9 and $(3 R, 5 R)$-5-(tert-Butyldiphenylsilanyloxymethyl)-3-ethylhept-1-ene-3,5-diol 8:

Anhydrous $\mathrm{CeCl}_{3}(36.7 \mathrm{mmol}, 9.04 \mathrm{~g})$ was ground with a pestle and mortar to a fine powder and added to a $500-\mathrm{mL}$, oven-dried, three-necked, round-bottomed flask equipped with a magnetic stirring bar. The flask was placed under vacuum and heated to $140{ }^{\circ} \mathrm{C}$ for $2 \mathrm{~h}$. The flask was filled with argon while hot, and cooled to $23{ }^{\circ} \mathrm{C}$. Freshly distilled THF $(300 \mathrm{~mL})$ at a temperature of $23{ }^{\circ} \mathrm{C}$ (NOTE: Do not add warm THF directly from the still - allow it to cool) was added via 
syringe and the mixture stirred as vigorously as possible overnight until a white cloudy suspension formed. A low temperature thermometer was inserted, the suspension was cooled to $78{ }^{\circ} \mathrm{C}$ and vinylmagnesium chloride $(36.7 \mathrm{mmol}, 1.68 \mathrm{M}$ in THF, $21.8 \mathrm{~mL})$ was added via syringe. The flask was warmed to $-20^{\circ} \mathrm{C}$ and stirred for $1 \mathrm{~h}$ until the solution turned deep red. The reaction mixture was cooled to $-78^{\circ} \mathrm{C}$ and 7 (4.89 g, $12.2 \mathrm{mmol}$ in $25 \mathrm{~mL}$ THF) was added dropwise. The reaction was warmed to $0{ }^{\circ} \mathrm{C}$ and stirred for $1 \mathrm{~h}$. Saturated $\mathrm{NH}_{4} \mathrm{Cl}(100 \mathrm{~mL})$ was added, and the reaction warmed to $23{ }^{\circ} \mathrm{C}$. The mixture was transferred to a separatory funnel and diluted with ether $(500 \mathrm{~mL})$. The layers were separated, and the aqueous layer extracted with ether $(3 \times 50 \mathrm{~mL})$. The combined organic layers were washed with saturated brine $(100 \mathrm{~mL})$ and water $(100 \mathrm{~mL})$, dried over $\mathrm{MgSO}_{4}$, filtered and the solvent removed by rotary evaporation. The resultant yellow oil, a mixture of two diastereomers, was purified by preparative HPLC $\left(\mathrm{Sil} \mathrm{SiO}_{2}\right.$ column, 24 x 0.46mm, 9:1 hexanes:MTBE) to yield the isomers $8(2.30 \mathrm{~g}, 44 \%)$ and 9 (2.51 g, $48 \%$ ), $\mathrm{R}_{\mathrm{f}} 0.20$ and 0.22 (hexanes/ether 9:1).

Characterization of (3R,5R)-5-(tert-Butyldiphenylsilanyloxymethyl)-3-ethylhept-1-ene-3,5diol 8: ${ }^{1} \mathrm{H}$ NMR $\left(400 \mathrm{MHz}, \mathrm{CDCl}_{3}\right) \delta 0.68\left(\mathrm{t}, \mathrm{J}=7.5 \mathrm{~Hz}, 3 \mathrm{H}, \mathrm{H}_{1}\right.$ or $\left.\mathrm{H}_{7}\right) ; 0.80(\mathrm{t}, \mathrm{J}=7.5 \mathrm{~Hz}, 3 \mathrm{H}$, $\mathrm{H}_{1}$ or $\left.\mathrm{H}_{7}\right) ; 1.06\left(\mathrm{~s}, 9 \mathrm{H}, \mathrm{H}_{16}\right) ; 1.41\left(\mathrm{dq}, \mathrm{J}=7.5,13.5 \mathrm{~Hz}, 1 \mathrm{H}, \mathrm{H}_{2}\right) ; 1.49$ (dq, J = 7.5, $13.5 \mathrm{~Hz}, 1 \mathrm{H}$, $\mathrm{H}_{2}$ ); 1.61 (dq, J = 7.5, 13.5 Hz, 1H, $\left.\mathrm{H}_{6}\right) ; 1.65$ (d, J = 15.0 Hz, 1H, $\left.\mathrm{H}_{4}\right) ; 1.70$ (dq, J = 7.5, $13.5 \mathrm{~Hz}$, $\left.1 \mathrm{H}, \mathrm{H}_{6}\right) ; 1.72\left(\mathrm{~d}, \mathrm{~J}=15.0 \mathrm{~Hz}, 1 \mathrm{H}, \mathrm{H}_{4}\right) ; 2.86(\mathrm{br} \mathrm{s}, 1 \mathrm{H}, \mathrm{OH}) ; 3.46\left(\mathrm{~d}, \mathrm{~J}=10.0 \mathrm{~Hz}, 1 \mathrm{H}, \mathrm{H}_{10}\right) ; 3.50$ $\left(\mathrm{d}, \mathrm{J}=10.0 \mathrm{~Hz}, 1 \mathrm{H}, \mathrm{H}_{10}\right) ; 4.34(\mathrm{br} \mathrm{s}, 1 \mathrm{H}, \mathrm{OH}) ; 5.13\left(\mathrm{dd}, \mathrm{J}=1.6,10.4 \mathrm{~Hz}, 1 \mathrm{H}, \mathrm{H}_{9}\right) ; 5.38(\mathrm{dd}, \mathrm{J}=$ 1.6, 17.2 Hz, 1H, $\left.\mathrm{H}_{9}\right) ; 5.92\left(\mathrm{dd}, \mathrm{J}=10.4,17.2 \mathrm{~Hz}, 1 \mathrm{H}, \mathrm{H}_{8}\right)$; 7.38-7.48 (m, 6H, $\left.\mathrm{H}_{13}, \mathrm{H}_{14}\right)$; 7.62$7.66\left(\mathrm{~m}, 4 \mathrm{H}, \mathrm{H}_{12}\right) .{ }^{13} \mathrm{C}$ NMR $\left(125 \mathrm{MHz}, \mathrm{CDCl}_{3}\right) \delta 7.7\left(\mathrm{C}_{7}\right) ; 8.4\left(\mathrm{C}_{7}\right) ; 19.5\left(\mathrm{C}_{15}\right) ; 27.1\left(\mathrm{C}_{16}\right) ; 30.1$ $\left(\mathrm{C}_{2}\right) ; 36.6\left(\mathrm{C}_{6}\right) ; 43.3\left(\mathrm{C}_{4}\right) ; 69.4\left(\mathrm{C}_{5}\right.$ or $\left.\mathrm{C}_{3}\right) ; 75.5\left(\mathrm{C}_{5}\right.$ or $\left.\mathrm{C}_{3}\right)$; $76.7\left(\mathrm{C}_{10}\right) ; 112.7\left(\mathrm{C}_{9}\right) ; 128.0\left(\mathrm{C}_{13}\right)$; $130.1\left(\mathrm{C}_{14}\right) ; 135.8\left(\mathrm{C}_{12}\right) ; 145.0\left(\mathrm{C}_{11}\right) ; 144.9\left(\mathrm{C}_{8}\right)$ : IR $\left(\mathrm{NaCl}\right.$, thin film) $\tilde{v}_{\max }\left(\mathrm{cm}^{-1}\right) ; 701,998$,

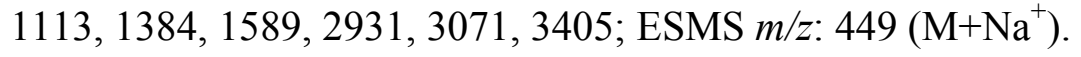

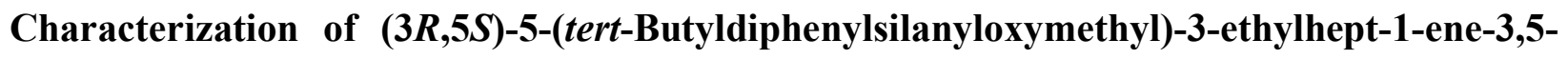
diol 9: ${ }^{1} \mathrm{H} \mathrm{NMR}\left(500 \mathrm{MHz}, \mathrm{CDCl}_{3}\right) \delta 0.82\left(\mathrm{t}, \mathrm{J}=7.5 \mathrm{~Hz}, 3 \mathrm{H}, \mathrm{H}_{1}\right.$ or $\left.\mathrm{H}_{7}\right) ; 0.83(\mathrm{t}, \mathrm{J}=7.5 \mathrm{~Hz}, 3 \mathrm{H}$, $\mathrm{H}_{1}$ or $\left.\mathrm{H}_{7}\right) ; 1.08$ (s, 9H, $\left.\mathrm{H}_{16}\right) ; 1.41$ (dq, J = 7.5, $\left.13.5 \mathrm{~Hz}, 1 \mathrm{H}, \mathrm{H}_{2}\right) ; 1.53$ (dq, J = 7.5, $13.5 \mathrm{~Hz}, 1 \mathrm{H}$,

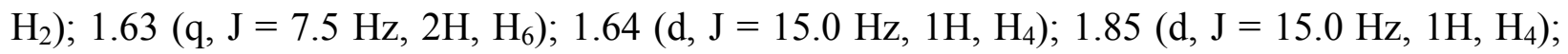


$2.82($ br s, $1 \mathrm{H}, \mathrm{OH}) ; 3.43\left(\mathrm{~d}, \mathrm{~J}=10.0 \mathrm{~Hz}, 1 \mathrm{H}, \mathrm{H}_{10}\right) ; 3.87\left(\mathrm{~d}, \mathrm{~J}=10.0 \mathrm{~Hz}, 1 \mathrm{H}, \mathrm{H}_{10}\right) ; 4.20(\mathrm{br} \mathrm{s}, 1 \mathrm{H}$, $\mathrm{OH}) ; 4.89\left(\mathrm{dd}, \mathrm{J}=1.5,10.5 \mathrm{~Hz}, 1 \mathrm{H}, \mathrm{H}_{9}\right) ; 5.22\left(\mathrm{dd}, \mathrm{J}=1.5,17.0 \mathrm{~Hz}, 1 \mathrm{H}, \mathrm{H}_{9}\right) ; 5.67(\mathrm{dd}, \mathrm{J}=10.5$, $\left.17.0 \mathrm{~Hz}, 1 \mathrm{H}, \mathrm{H}_{8}\right) ; 7.38-7.48\left(\mathrm{~m}, 6 \mathrm{H}, \mathrm{H}_{13}, \mathrm{H}_{14}\right) ; 7.62-7.66\left(\mathrm{~m}, 4 \mathrm{H}, \mathrm{H}_{12}\right) ;{ }^{13} \mathrm{C} \mathrm{NMR}(125 \mathrm{MHz}$, $\left.\mathrm{CDCl}_{3}\right) \delta 7.9\left(\mathrm{C}_{7}\right) ; 8.3\left(\mathrm{C}_{7}\right) ; 19.8\left(\mathrm{C}_{15}\right) ; 27.4\left(\mathrm{C}_{16}\right) ; 33.1\left(\mathrm{C}_{2}\right) ; 36.7\left(\mathrm{C}_{6}\right) ; 43.1\left(\mathrm{C}_{4}\right) ; 67.7\left(\mathrm{C}_{5}\right.$ or $\left.\mathrm{C}_{3}\right) ; 75.9\left(\mathrm{C}_{5}\right.$ or $\left.\mathrm{C}_{3}\right) ; 77.3\left(\mathrm{C}_{10}\right) ; 112.9\left(\mathrm{C}_{9}\right) ; 128.2\left(\mathrm{C}_{13}\right) ; 130.3\left(\mathrm{C}_{14}\right) ; 136.1\left(\mathrm{C}_{12}\right) ; 145.0\left(\mathrm{C}_{11}\right)$; 149.4 $\left(\mathrm{C}_{8}\right)$ : IR $\left(\mathrm{NaCl}\right.$, thin film) $\tilde{v}_{\max }\left(\mathrm{cm}^{-1}\right) ; 1085,1428,1472,1558,1652,2930$, 3356; EIMS

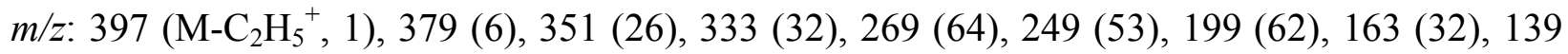
(100), 105 (68), 77 (61); ESMS m/z: $449\left(\mathrm{M}+\mathrm{Na}^{+}\right)$.

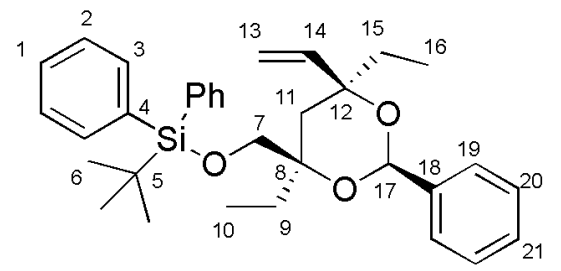

S-3

Preparation of $\quad(2 S, 4 S, 6 R)$-tert-Butyl(4,6-diethyl-2-phenyl-6-vinyl-1,3-dioxan-4ylmethoxy)diphenylsilane S-3 and (2R,4S,6R)-tert-Butyl(4,6-diethyl-2-phenyl-6-vinyl-1,3dioxan-4-ylmethoxy)diphenylsilane S-4: To a $250 \mathrm{~mL}$, three-necked, round-bottomed flask equipped with a magnetic stirring bar was added $9(720 \mathrm{mg}, 1.70 \mathrm{mmol})$. The flask was placed under an argon atmosphere, and $\mathrm{CH}_{2} \mathrm{Cl}_{2}(150 \mathrm{~mL})$ was added via syringe. The mixture was cooled to $0{ }^{\circ} \mathrm{C}$ and benzaldehyde dimethyl acetal $(388 \mathrm{mg}, 382 \mu \mathrm{L}, 2.55 \mathrm{mmol})$ was added via syringe. A crystal of $p$-toluenesulfonic acid $(\sim 5 \mathrm{mg})$ was added and the reaction slowly warmed to $23{ }^{\circ} \mathrm{C}$, then stirred for $5 \mathrm{~h}$. The solution was filtered through a short silica pad and the solvent removed by rotary evaporation. The resulting yellow oil was purified by column chromatography $\left(\mathrm{SiO}_{2}\right.$ : hexanes/ether 50:1) to yield the two diastereomers, S-3 (513 mg, 59\%) and S-4 (342 mg, $39 \%$ ), epimeric at $\mathrm{C}_{17}$ as colorless oils, $\mathrm{R}_{\mathrm{f}} 0.85,0.82$ (hexanes/ether 19:1) respectively.

Characterization of $(2 S, 4 S, 6 R)-t e r t-B u t y l(4,6-d i e t h y l-2-p h e n y l-6-v i n y l-1,3-d i o x a n-4-$ ylmethoxy)diphenylsilane S-3: ${ }^{1} \mathrm{H}$ NMR $\left(500 \mathrm{MHz}, \mathrm{C}_{6} \mathrm{D}_{6}, \delta\right.$, ): $0.87\left(\mathrm{t}, \mathrm{J}=7.5 \mathrm{~Hz}, 3 \mathrm{H}, \mathrm{H}_{16}\right)$, 0.89 (t, J = $\left.7.5 \mathrm{~Hz}, 3 \mathrm{H}, \mathrm{H}_{16}\right) ; 1.18\left(\mathrm{~s}, 9 \mathrm{H}, \mathrm{H}_{6}\right) ; 1.33$ (dq, J = 7.5, $14.0 \mathrm{~Hz}, 1 \mathrm{H}, \mathrm{H}_{9}$ or $\mathrm{H}_{15}$ ); 1.64 (d, $\left.\mathrm{J}=15.0 \mathrm{~Hz}, 1 \mathrm{H}, \mathrm{H}_{11}\right) ; 1.69\left(\mathrm{dq}, \mathrm{J}=7.5,14.0 \mathrm{~Hz}, 1 \mathrm{H}, \mathrm{H}_{9}\right.$ or $\left.\mathrm{H}_{15}\right) ; 1.80(\mathrm{dq}, \mathrm{J}=7.5,14.0 \mathrm{~Hz}, 1 \mathrm{H}$, 
$\mathrm{H}_{9}$ or $\left.\mathrm{H}_{15}\right) ; 1.82\left(\mathrm{dq}, \mathrm{J}=7.5,14.0 \mathrm{~Hz}, 1 \mathrm{H}, \mathrm{H}_{9}\right.$ or $\left.\mathrm{H}_{15}\right) ; 2.03\left(\mathrm{~d}, \mathrm{~J}=15.0 \mathrm{~Hz}, 1 \mathrm{H}, \mathrm{H}_{11}\right) ; 3.77(\mathrm{~d}, \mathrm{~J}=$ $\left.10.0 \mathrm{~Hz}, 1 \mathrm{H}, \mathrm{H}_{7}\right) ; 3.94\left(\mathrm{~d}, \mathrm{~J}=10.0 \mathrm{~Hz}, 1 \mathrm{H}, \mathrm{H}_{7}\right) ; 4.91\left(\mathrm{dd}, \mathrm{J}=2.5,10.5 \mathrm{~Hz}, 1 \mathrm{H}, \mathrm{H}_{13}\right) ; 5.43$ (dd, J $\left.=2.5,17.5 \mathrm{~Hz}, 1 \mathrm{H}, \mathrm{H}_{13}\right) ; 5.53\left(\mathrm{dd}, \mathrm{J}=10.5,17.5 \mathrm{~Hz}, 1 \mathrm{H}, \mathrm{H}_{14}\right) ; 5.88\left(\mathrm{~s}, 1 \mathrm{H}, \mathrm{H}_{17}\right) ; 7.10-7.20(\mathrm{~m}$, $\left.9 \mathrm{H}, \mathrm{H}_{1}, \mathrm{H}_{2}, \mathrm{H}_{20}, \mathrm{H}_{21}\right) ; 7.65\left(\mathrm{~d}, \mathrm{~J}=7.0 \mathrm{~Hz}, 2 \mathrm{H}, \mathrm{H}_{3}\right) ; 7.74-7.76\left(\mathrm{~m}, 4 \mathrm{H}, \mathrm{H}_{19}\right) ;{ }^{13} \mathrm{C} \mathrm{NMR}(125 \mathrm{MHz}$, $\left.\mathrm{C}_{6} \mathrm{D}_{6}\right) \delta 7.8\left(\mathrm{C}_{10}\right.$ or $\left.\mathrm{C}_{16}\right) ; 8.1\left(\mathrm{C}_{10}\right.$ or $\left.\mathrm{C}_{16}\right) ; 19.3\left(\mathrm{C}_{5}\right) ; 27.6\left(\mathrm{C}_{6}\right) ; 30.0\left(\mathrm{C}_{9}\right) ; 33.7\left(\mathrm{C}_{15}\right) ; 37.2\left(\mathrm{C}_{11}\right)$; $67.5\left(\mathrm{C}_{7}\right) ; 76.8\left(\mathrm{C}_{8}\right) ; 77.5\left(\mathrm{C}_{12}\right) ; 91.5\left(\mathrm{C}_{11}\right) ; 113.9\left(\mathrm{C}_{13}\right) ; 127.2\left(\mathrm{C}_{21}\right) ; 127.9\left(\mathrm{C}_{19}\right) ; 128.8\left(\mathrm{C}_{20}\right)$; $130.4\left(\mathrm{C}_{2}\right) ; 134.3\left(\mathrm{C}_{1}\right) ; 134.5\left(\mathrm{C}_{4}\right) ; 136.6\left(\mathrm{C}_{3}\right) ; 141.2\left(\mathrm{C}_{18}\right) ; 144.2\left(\mathrm{C}_{14}\right)$; EIHRMS m/z: calcd for

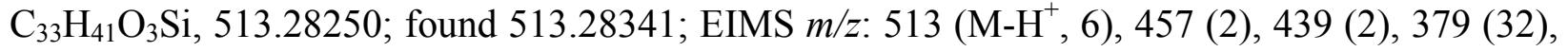
351 (51), 301 (16), 269 (68), 239 (38), 191 (60), 139 (100), 105, (65), 77 (68).

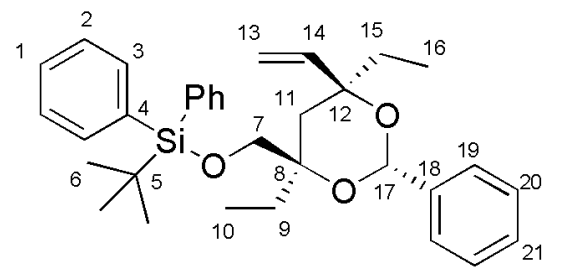

S-4

Characterization of $(2 R, 4 S, 6 R)$-tert-Butyl(4,6-diethyl-2-phenyl-6-vinyl-1,3-dioxan-4ylmethoxy)diphenylsilane S-4: ${ }^{1} \mathrm{H}$ NMR $\left(500 \mathrm{MHz}, \mathrm{C}_{6} \mathrm{D}_{6}\right) \delta 0.85\left(\mathrm{t}, \mathrm{J}=7.5 \mathrm{~Hz}, 3 \mathrm{H}, \mathrm{H}_{16}\right), 0.99$ $\left(\mathrm{t}, \mathrm{J}=7.5 \mathrm{~Hz}, 3 \mathrm{H}, \mathrm{H}_{16}\right) ; 1.12\left(\mathrm{~s}, 9 \mathrm{H}, \mathrm{H}_{6}\right) ; 1.48\left(\mathrm{dq}, \mathrm{J}=7.5,14.0 \mathrm{~Hz}, 1 \mathrm{H}, \mathrm{H}_{9}\right.$ or $\left.\mathrm{H}_{15}\right) ; 1.61$ (dq, J = 7.5, $14.0 \mathrm{~Hz}, 1 \mathrm{H}, \mathrm{H}_{9}$ or $\left.\mathrm{H}_{15}\right) ; 1.64$ (d, J = $\left.15.0 \mathrm{~Hz}, 1 \mathrm{H}, \mathrm{H}_{11}\right) ; 1.69$ (d, J = $\left.15.0 \mathrm{~Hz}, 1 \mathrm{H}, \mathrm{H}_{11}\right) ; 1.89$ $\left(\mathrm{dq}, \mathrm{J}=7.5,14.0 \mathrm{~Hz}, 1 \mathrm{H}, \mathrm{H}_{9}\right.$ or $\left.\mathrm{H}_{15}\right) ; 1.97\left(\mathrm{dq}, \mathrm{J}=7.5,14.0 \mathrm{~Hz}, 1 \mathrm{H}, \mathrm{H}_{9}\right.$ or $\left.\mathrm{H}_{15}\right) ; 3.86(\mathrm{~d}, \mathrm{~J}=10.0$ $\left.\mathrm{Hz}, 1 \mathrm{H}, \mathrm{H}_{7}\right) ; 4.03\left(\mathrm{~d}, \mathrm{~J}=10.0 \mathrm{~Hz}, 1 \mathrm{H}, \mathrm{H}_{7}\right) ; 4.88\left(\mathrm{dd}, \mathrm{J}=1.5,11.0 \mathrm{~Hz}, 1 \mathrm{H}, \mathrm{H}_{13}\right) ; 5.12$ (dd, J = 1.5, $\left.17.5 \mathrm{~Hz}, 1 \mathrm{H}, \mathrm{H}_{13}\right) ; 5.44$ (dd, J = 11.0, 17.5 Hz, 1H, H$\left.{ }_{14}\right) ; 5.85\left(\mathrm{~s}, 1 \mathrm{H}, \mathrm{H}_{17}\right) ; 7.11-7.24\left(\mathrm{~m}, 9 \mathrm{H}, \mathrm{H}_{1}\right.$, $\left.\mathrm{H}_{2}, \mathrm{H}_{20}, \mathrm{H}_{21}\right) ; 7.65\left(\mathrm{~d}, \mathrm{~J}=7.0 \mathrm{~Hz}, 2 \mathrm{H}, \mathrm{H}_{3}\right) ; 7.70-7.78\left(\mathrm{~m}, 4 \mathrm{H}, \mathrm{H}_{19}\right) ;{ }^{13} \mathrm{C} \mathrm{NMR}\left(125 \mathrm{MHz}, \mathrm{C}_{6} \mathrm{D}_{6}\right) \delta$ $7.0\left(\mathrm{C}_{10}\right.$ or $\left.\mathrm{C}_{16}\right)$; $7.3\left(\mathrm{C}_{10}\right.$ or $\left.\mathrm{C}_{16}\right) ; 19.4\left(\mathrm{C}_{5}\right) ; 27.0\left(\mathrm{C}_{6}\right) ; 32.7\left(\mathrm{C}_{9}\right) ; 36.9\left(\mathrm{C}_{15}\right) ; 37.7\left(\mathrm{C}_{11}\right) ; 62.9\left(\mathrm{C}_{7}\right)$; $76.1\left(\mathrm{C}_{8}\right) ; 76.3\left(\mathrm{C}_{12}\right) ; 90.3\left(\mathrm{C}_{11}\right) ; 114.9\left(\mathrm{C}_{13}\right) ; 126.7\left(\mathrm{C}_{21}\right) ; 127.5\left(\mathrm{C}_{19}\right) ; 128.3\left(\mathrm{C}_{20}\right) ; 130.0\left(\mathrm{C}_{2}\right)$; $133.6\left(\mathrm{C}_{1}\right) ; 134.0\left(\mathrm{C}_{4}\right) ; 136.1\left(\mathrm{C}_{3}\right) ; 140.5\left(\mathrm{C}_{18}\right) ; 141.5\left(\mathrm{C}_{14}\right)$; EIMS m/z: $513\left(\mathrm{M}-\mathrm{H}^{+}, 6\right), 457(2)$, 379 (41); 351 (49); 303 (51); 269 (82); 239 (52); 191 (66); 163 (72); 139 (100); 77 (68); EIHRMS $m / z$ : calcd for $\mathrm{C}_{33} \mathrm{H}_{41} \mathrm{O}_{3} \mathrm{Si}, 513.28250$; found 513.28207. 


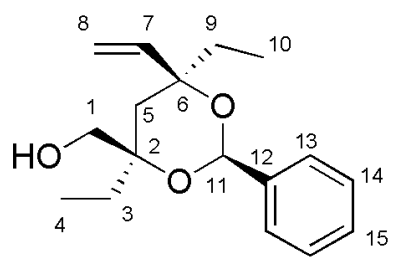

S-5

Preparation of (4S,6R)-(4,6-Diethyl-2-phenyl-6-vinyl-1,3-dioxan-4-yl)methanol S-5: To a 25

$\mathrm{mL}$, three-necked, round-bottomed flask equipped with a magnetic stirbar and reflux condenser was added S-3 (890 mg, $1.74 \mathrm{mmol})$. The flask was placed under an argon atmosphere, and THF $(5 \mathrm{~mL})$ was added via syringe. Tetrabutylammonium fluoride (5.21 mmol, $1 \mathrm{M}$ in THF, $5.21 \mathrm{~mL})$ was added via syringe and the mixture heated to $50{ }^{\circ} \mathrm{C}$, then stirred for $3 \mathrm{~h}$. The flask was cooled to $23{ }^{\circ} \mathrm{C}$ and saturated $\mathrm{NH}_{4} \mathrm{Cl}(20 \mathrm{~mL})$ added. The mixture was transferred to a separatory flask, diluted with ether $(50 \mathrm{~mL})$, and the layers separated. The aqueous layer was extracted with ether $(3 \mathrm{x} 10 \mathrm{~mL})$ and the combined organic extracts washed with saturated brine $(20 \mathrm{~mL})$ and water $(20 \mathrm{~mL})$, dried over $\mathrm{MgSO}_{4}$, filtered and the solvent removed by rotary evaporation. The crude oil was purified by column chromatography $\left(\mathrm{SiO}_{2}\right.$ : hexanes/ether 4:1) to yield $\mathbf{S - 5}$ as a colorless oil containing a small (10\%), inseparable amount of the epimer at $\mathrm{C}_{11}(451 \mathrm{mg}, 94 \%)$ as a colorless oil, $\mathrm{R}_{\mathrm{f}} 0.20$ (hexanes/ether 4:1); ${ }^{1} \mathrm{H}$ NMR $\left(500 \mathrm{MHz}, \mathrm{C}_{6} \mathrm{D}_{6}\right.$, major isomer) $\delta 0.83(\mathrm{t}, \mathrm{J}=$ $\left.7.5 \mathrm{~Hz}, 3 \mathrm{H}, \mathrm{H}_{10}\right) ; 0.89$ (t, J = 7.5 Hz, 3H, H4); 1.41 (d, J = 14.0 Hz, H ); 1.47 (dq, J = 7.5, 13.5 $\mathrm{Hz}, 1 \mathrm{H}, \mathrm{H}_{9}$ or $\left.\mathrm{H}_{3}\right) ; 1.57$ (dq, J = 7.5, $13.5 \mathrm{~Hz}, 1 \mathrm{H}, \mathrm{H}_{9}$ or $\left.\mathrm{H}_{3}\right) ; 1.58\left(\mathrm{~d}, \mathrm{~J}=14.0 \mathrm{~Hz}, \mathrm{H}_{5}\right) ; 1.69 \mathrm{dq}, \mathrm{J}$ $=7.5,13.5 \mathrm{~Hz}, 1 \mathrm{H}, \mathrm{H}_{9}$ or $\left.\mathrm{H}_{3}\right) ; 1.71\left(\mathrm{dq}, \mathrm{J}=7.5,13.5 \mathrm{~Hz}, 1 \mathrm{H}, \mathrm{H}_{9}\right.$ or $\left.\mathrm{H}_{3}\right) ; 3.38(\mathrm{dd}, \mathrm{J}=8.0,11.0$ $\left.\mathrm{Hz}, 1 \mathrm{H}, \mathrm{H}_{1}\right) ; 3.89\left(\mathrm{~d}, \mathrm{~J}=11.0 \mathrm{~Hz}, 1 \mathrm{H}, \mathrm{H}_{1}\right) ; 5.06\left(\mathrm{dd}, \mathrm{J}=1.5,11.0 \mathrm{~Hz}, 1 \mathrm{H}, \mathrm{H}_{8}\right) ; 5.15$ (dd, J = 1.5, $\left.18.0 \mathrm{~Hz}, 1 \mathrm{H}, \mathrm{H}_{8}\right) ; 5.55\left(\mathrm{dd}, \mathrm{J}=11.0,18.0 \mathrm{~Hz}, 1 \mathrm{H}, \mathrm{H}_{7}\right) ; 5.86\left(\mathrm{~s}, 1 \mathrm{H}, \mathrm{H}_{11}\right) ; 7.11-7.22\left(\mathrm{~m}, 3 \mathrm{H}, \mathrm{H}_{14}\right.$, $\left.\mathrm{H}_{15}\right) ; 7.62-7.65\left(\mathrm{~m}, 2 \mathrm{H}, \mathrm{H}_{13}\right) ;{ }^{13} \mathrm{C} \mathrm{NMR}\left(125 \mathrm{MHz}, \mathrm{C}_{6} \mathrm{D}_{6}\right) \delta 7.0\left(\mathrm{C}_{10}\right.$ or $\left.\mathrm{C}_{4}\right) ; 7.3\left(\mathrm{C}_{10}\right.$ or $\left.\mathrm{C}_{4}\right) ; 32.1$ $\left(\mathrm{C}_{3}\right) ; 36.3\left(\mathrm{C}_{9}\right) ; 37.8\left(\mathrm{C}_{5}\right) ; 61.4\left(\mathrm{C}_{1}\right) ; 76.1\left(\mathrm{C}_{6}\right) ; 76.6\left(\mathrm{C}_{2}\right) ; 90.6\left(\mathrm{C}_{11}\right) ; 115.1\left(\mathrm{C}_{7}\right) ; 126.8\left(\mathrm{C}_{15}\right)$;

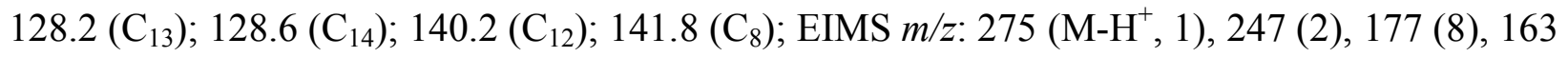
(6), 139 (100), 123 (38), 105 (78), 95 (38), 77 (88), 67 (55); IR (NaCl, thin film) $\tilde{v}_{\max }\left(\mathrm{cm}^{-1}\right)$; 924, 1089, 1175, 1214, 1383, 1457, 2881, 3441; EIHRMS $m / z$ : calcd for $\mathrm{C}_{17} \mathrm{H}_{23} \mathrm{O}_{3}, 275.16472$; found 275.16420 . 


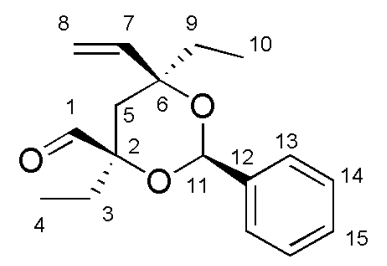

10

Preparation of (4S,6R)-4,6-Diethyl-2-phenyl-6-vinyl-1,3-dioxane-4-carbaldehyde 10: To an oven-dried, 100-mL, two-necked flask equipped with a magnetic stirring bar was added $4 \AA$ molecular sieves $(450 \mathrm{mg})$ and pyridinium chlorochromate $(2.01 \mathrm{mmol}, 433 \mathrm{mg})$. The flask was placed under an argon atmosphere and $\mathrm{CH}_{2} \mathrm{Cl}_{2}(30 \mathrm{~mL})$ was added via syringe. One drop of a $1 \%$ acetic acid solution in $\mathrm{CH}_{2} \mathrm{Cl}_{2}$ was added, and the mixture cooled to $0{ }^{\circ} \mathrm{C}$. Alcohol $\mathbf{S}-5$ (370 mg, $1.34 \mathrm{mmol})$ in $\mathrm{CH}_{2} \mathrm{Cl}_{2}(5 \mathrm{~mL})$ was added dropwise $(5 \mathrm{~min})$ and the mixture warmed to $23{ }^{\circ} \mathrm{C}$ over a period of $3 \mathrm{~h}$. The reaction was monitored by tlc until complete $(11 \mathrm{~h})$. Ether $(50 \mathrm{~mL})$ was added and the resulting brown slurry filtered through a pad of Celite with a monolayer of silica on the top. The solvent was removed by rotary evaporation, ether $(50 \mathrm{~mL})$ added and the above filtration repeated. The solvent was again removed by rotary evaporation to yield a yellow oil, which was purified by column chromatography $\left(\mathrm{SiO}_{2}\right.$ : hexanes/ether 19:1) to yield 10 (304 mg, $83 \%)$ as a pale yellow oil containing a small $(<5 \%)$, inseparable amount of the epimer at $C_{11}, R_{f}$ 0.70 (hexanes/ether 9:1); ${ }^{1} \mathrm{H} \mathrm{NMR}\left(500 \mathrm{MHz}, \mathrm{CDCl}_{3}, \delta\right.$, major isomer): $0.91(\mathrm{t}, \mathrm{J}=7.5 \mathrm{~Hz}, 3 \mathrm{H}$, $\left.\mathrm{H}_{10}\right) ; 0.94\left(\mathrm{t}, \mathrm{J}=7.5 \mathrm{~Hz}, 3 \mathrm{H}, \mathrm{H}_{4}\right) ; 1.60-1.78\left(\mathrm{~m}, 4 \mathrm{H}, \mathrm{H}_{3}, \mathrm{H}_{9}\right) ; 1.72\left(\mathrm{~d}, \mathrm{~J}=13.5 \mathrm{~Hz}, 1 \mathrm{H}, \mathrm{H}_{5}\right) ; 2.33$ $\left(\mathrm{d}, \mathrm{J}=13.5 \mathrm{~Hz}, 1 \mathrm{H}, \mathrm{H}_{5}\right) ; 5.18\left(\mathrm{dd}, \mathrm{J}=1.5,18.0 \mathrm{~Hz}, 1 \mathrm{H}, \mathrm{H}_{8}\right) ; 5.31$ (dd, J = 1.5, $\left.11.0 \mathrm{~Hz}, 1 \mathrm{H}, \mathrm{H}_{8}\right)$; $5.68\left(\mathrm{dd}, \mathrm{J}=11.0,18.0 \mathrm{~Hz}, 1 \mathrm{H}, \mathrm{H}_{7}\right) ; 5.86\left(\mathrm{~s}, 1 \mathrm{H}, \mathrm{C}_{11}\right) ; 7.35-7.44\left(\mathrm{~m}, 3 \mathrm{H}, \mathrm{H}_{14}, \mathrm{H}_{15}\right) ; 7.58-7.64(\mathrm{~m}$, $\left.2 \mathrm{H}, \mathrm{H}_{13}\right) ; 9.79\left(\mathrm{~d}, \mathrm{~J}=1.0 \mathrm{~Hz}, \mathrm{H}_{1}\right) ;{ }^{13} \mathrm{C} \mathrm{NMR}\left(125 \mathrm{MHz}, \mathrm{CDCl}_{3}\right) \delta 6.8\left(\mathrm{C}_{4}\right) ; 7.2\left(\mathrm{C}_{10}\right) ; 31.6\left(\mathrm{C}_{3}\right)$; $37.2\left(\mathrm{C}_{9}\right) ; 37.4\left(\mathrm{C}_{5}\right) ; 77.8\left(\mathrm{C}_{6}\right) ; 82.1\left(\mathrm{C}_{2}\right) ; 93.3\left(\mathrm{C}_{11}\right) ; 117.3\left(\mathrm{C}_{8}\right) ; 126.4\left(\mathrm{C}_{15}\right) ; 128.5\left(\mathrm{C}_{13}\right) ; 129.1$ $\left(\mathrm{C}_{14}\right) ; 138.9\left(\mathrm{C}_{12}\right) ; 140.1\left(\mathrm{C}_{7}\right) ; 205.4\left(\mathrm{C}_{1}\right)$; EIMS m/z: $273\left(\mathrm{M}-\mathrm{H}^{+}, 1\right), 245$ (5), 163 (8), 139 (100), 113 (42), 105 (80), 77 (55); EIHRMS m/z: calcd for $\mathrm{C}_{17} \mathrm{H}_{21} \mathrm{O}_{3}, 273.14907$; found 273.14877; IR (NaCl, thin film) $\tilde{v}_{\max }\left(\mathrm{cm}^{-1}\right) ; 746,1015,1175,1215,1383,1727,2941$. 


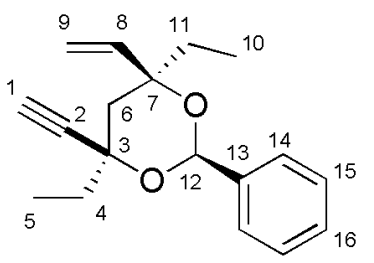

S-6

Preparation of (4S,6R)-4,6-Diethyl-4-ethynyl-2-phenyl-6-vinyl-1,3-dioxane S-6: To a 50$\mathrm{mL}$, three-necked, round-bottomed flask equipped with a magnetic stirring bar was added anhydrous potassium carbonate $(3.56 \mathrm{mmol}, 491 \mathrm{mg})$ The flask was placed under an argon atmosphere, and aldehyde 10 (480 mg, $1.78 \mathrm{mmol})$ in $\mathrm{MeOH}(15 \mathrm{~mL})$ was added via syringe. Dimethyl-1-diazo-2-oxopropylphosphonate (2.67 mmol, $512 \mathrm{mg}$, in $2 \mathrm{~mL} \mathrm{MeOH}$ ) was added dropwise via syringe. The mixture was stirred at for $15 \mathrm{~h}$ then diluted with $\mathrm{Et}_{2} \mathrm{O}(50 \mathrm{~mL})$. Saturated $\mathrm{NaHCO}_{3}(15 \mathrm{~mL})$ was added and the mixture was transferred to a separatory flask. The layers were separated and the aqueous layer was extracted with ether ( 3 x $10 \mathrm{~mL})$. The combined organic extracts were washed with saturated brine $(20 \mathrm{~mL})$ and water $(20 \mathrm{~mL})$, dried over $\mathrm{MgSO}_{4}$, filtered and the solvent removed by rotary evaporation. The crude oil was purified by column chromatography $\left(\mathrm{SiO}_{2}\right.$ : hexanes/ether 50:1) to yield acetylene $\mathbf{S - 6}(350 \mathrm{mg}, 74 \%)$ as a colorless oil, $\mathrm{R}_{\mathrm{f}} 0.75$ (hexanes/ether 19:1); ${ }^{1} \mathrm{H}$ NMR (400 MHz, $\left.\mathrm{C}_{6} \mathrm{D}_{6}\right) \delta 0.87(\mathrm{t}, \mathrm{J}=7.2 \mathrm{~Hz}, 3 \mathrm{H}$, $\left.\mathrm{H}_{10}\right) ; 1.10\left(\mathrm{t}, \mathrm{J}=7.6 \mathrm{~Hz}, 3 \mathrm{H}, \mathrm{H}_{5}\right) ; 1.49\left(\mathrm{dq}, \mathrm{J}=5.7,13.2 \mathrm{~Hz}, 1 \mathrm{H}, \mathrm{H}_{11}\right.$ or $\left.\mathrm{H}_{4}\right) ; 1.62(\mathrm{dq}, \mathrm{J}=5.7$, $13.2 \mathrm{~Hz}, 1 \mathrm{H}, \mathrm{H}_{11}$ or $\left.\mathrm{H}_{4}\right) ; 1.62\left(\mathrm{~d}, \mathrm{~J}=13.2 \mathrm{~Hz}, 1 \mathrm{H}, \mathrm{H}_{6}\right) ; 1.65\left(\mathrm{dq}, \mathrm{J}=5.7,13.2 \mathrm{~Hz}, 1 \mathrm{H}, \mathrm{H}_{11}\right.$ or $\left.\mathrm{H}_{4}\right)$; $1.71\left(\mathrm{~d}, \mathrm{~J}=13.2 \mathrm{~Hz}, 1 \mathrm{H}, \mathrm{H}_{6}\right) ; 1.83\left(\mathrm{dq}, \mathrm{J}=5.7,13.2 \mathrm{~Hz}, 1 \mathrm{H}, \mathrm{H}_{11}\right.$ or $\left.\mathrm{H}_{4}\right) ; 2.09\left(\mathrm{~s}, 1 \mathrm{H}, \mathrm{H}_{1}\right) ; 5.25$ $\left(\mathrm{dd}, \mathrm{J}=1.6,11.2,1 \mathrm{H}, \mathrm{H}_{9}\right) ; 5.38\left(\mathrm{dd}, \mathrm{J}=1.6,17.2,1 \mathrm{H}, \mathrm{H}_{9}\right) ; 5.89\left(\mathrm{dd}, \mathrm{J}=11.2,17.2,1 \mathrm{H}, \mathrm{H}_{9}\right)$; $6.48\left(\mathrm{~s}, 1 \mathrm{H}, \mathrm{H}_{12}\right) ; 7.09-7.22\left(\mathrm{~m}, 3 \mathrm{H}, \mathrm{H}_{15}+\mathrm{H}_{16}\right) ; 7.72-7.76\left(\mathrm{~m}, 2 \mathrm{H}, \mathrm{H}_{14}\right) ;{ }^{13} \mathrm{C} \mathrm{NMR}(125 \mathrm{MHz}$, $\left.\mathrm{C}_{6} \mathrm{D}_{6}\right) \delta 6.9\left(\mathrm{C}_{5}\right) ; 7.8\left(\mathrm{C}_{10}\right) ; 37.1\left(\mathrm{C}_{4}\right) ; 37.7\left(\mathrm{C}_{11}\right) ; 43.1\left(\mathrm{C}_{6}\right) ; 72.2\left(\mathrm{C}_{1}\right) ; 75.6\left(\mathrm{C}_{3}\right) ; 77.1\left(\mathrm{C}_{7}\right) ; 84.4$ $\left(\mathrm{C}_{2}\right)$; $92.6\left(\mathrm{C}_{12}\right) ; 115.4\left(\mathrm{C}_{9}\right) ; 126.8\left(\mathrm{C}_{14}\right) ; 128.2\left(\mathrm{C}_{16}\right) ; 128.5\left(\mathrm{C}_{15}\right) ; 139.9\left(\mathrm{C}_{13}\right) ; 140.8\left(\mathrm{C}_{8}\right)$; EIMS m/z: 269 (M-H+ , 2), 241 (4), 135 (50), 119 (62), 105 (100), 91 (42), 77 (46); EIHRMS m/z: calcd for $\mathrm{C}_{18} \mathrm{H}_{21} \mathrm{O}_{2}, 269.15415$; found 269.15405; IR (NaCl, thin film) $\tilde{v}_{\max }\left(\mathrm{cm}^{-1}\right) ; 920,1126,1383$, $1455,2972,3030,3291$. 


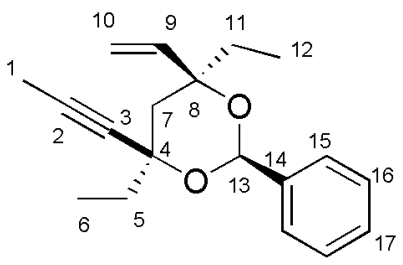

11

Preparation of (4S,6R)-4,6-Diethyl-2-phenyl-4-prop-1-ynyl-6-vinyl-1,3-dioxane 11: To a 25$\mathrm{mL}$, three-necked, round-bottomed flask equipped with a magnetic stirring bar was added S-6 (100 mg, $0.38 \mathrm{mmol})$. The flask was placed under an argon atmosphere, and THF (5 mL) was added via syringe. The mixture was cooled to $-78{ }^{\circ} \mathrm{C}$ and $n$-butyllithium $(0.38 \mathrm{mmol}, 1.38 \mathrm{M}$ solution in hexanes, $272 \mu \mathrm{L}$ ) was added dropwise via syringe. The mixture was stirred at $-78^{\circ} \mathrm{C}$ for $30 \mathrm{~min}$ and methyl trifluoromethanesulfonate $(0.56 \mathrm{mmol}, 92 \mathrm{mg}, 64 \mu \mathrm{L})$ was added dropwise via syringe. The mixture was stirred at $-78{ }^{\circ} \mathrm{C}$ for $1 \mathrm{~h}$, then saturated $\mathrm{NH}_{4} \mathrm{Cl}(5 \mathrm{~mL})$ was added. The mixture was warmed to $23{ }^{\circ} \mathrm{C}$, diluted with ether $(50 \mathrm{~mL})$, transferred to a separatory flask, and the layers separated. The aqueous layer was extracted with ether $(3 \mathrm{x} 10 \mathrm{~mL})$ and the combined organic extracts washed with saturated brine $(20 \mathrm{~mL})$ and water $(20 \mathrm{~mL})$, dried over $\mathrm{MgSO}_{4}$, filtered and the solvent removed by rotary evaporation. The crude oil was purified by column chromatography $\left(\mathrm{SiO}_{2}\right.$ : hexanes/ether 50:1) to yield 11 (101 mg, 96\%) as a colorless oil, $\mathrm{R}_{\mathrm{f}} 0.75$ (hexanes/ether 19:1); ${ }^{1} \mathrm{H}$ NMR $\left(500 \mathrm{MHz}, \mathrm{C}_{6} \mathrm{D}_{6}\right) \delta 0.87\left(\mathrm{t}, \mathrm{J}=7.2 \mathrm{~Hz}, 3 \mathrm{H}, \mathrm{H}_{12}\right) ; 1.17(\mathrm{t}, \mathrm{J}$ $\left.=7.6 \mathrm{~Hz}, 3 \mathrm{H}_{1} \mathrm{H}_{6}\right) ; 1.49\left(\mathrm{~s}, 3 \mathrm{H}, \mathrm{H}_{1}\right) 1.53\left(\mathrm{dq}, \mathrm{J}=7.5,13.0 \mathrm{~Hz}, 1 \mathrm{H}, \mathrm{H}_{5}\right.$ or $\left.\mathrm{H}_{11}\right) ; 1.64(\mathrm{dq}, \mathrm{J}=7.5$, $13.0 \mathrm{~Hz}, 1 \mathrm{H}, \mathrm{H}_{5}$ or $\left.\mathrm{H}_{11}\right) ; 1.68$ (d, J = 13.2 Hz, 1H, $\left.\mathrm{H}_{7}\right) ; 1.69$ (dq, J = 7.5, $13.0 \mathrm{~Hz}, 1 \mathrm{H}, \mathrm{H}_{5}$ or $\mathrm{H}_{11}$ ); $1.77\left(\mathrm{~d}, \mathrm{~J}=13.2 \mathrm{~Hz}, 1 \mathrm{H}, \mathrm{H}_{7}\right) ; 1.89$ (dq, J = 7.5, $13.0 \mathrm{~Hz}, 1 \mathrm{H}, \mathrm{H}_{5}$ or $\left.\mathrm{H}_{11}\right) ; 5.26(\mathrm{dd}, 1.5,11.0 \mathrm{~Hz}$, $\left.1 \mathrm{H}, \mathrm{H}_{10}\right) ; 5.38\left(\mathrm{dd}, \mathrm{J}=1.5,17.5 \mathrm{~Hz}, 1 \mathrm{H}, \mathrm{H}_{10}\right) ; 5.91\left(\mathrm{dd}, \mathrm{J}=11.0,17.5 \mathrm{~Hz}, 1 \mathrm{H}, \mathrm{H}_{9}\right) ; 6.53(\mathrm{~s}, 1 \mathrm{H}$, $\left.\mathrm{H}_{13}\right)$; 7.09-7.22 (m, 3H, $\left.\mathrm{H}_{16}, \mathrm{H}_{17}\right)$; 7.76-7.82 (m, 2H, $\left.\mathrm{H}_{15}\right) ;{ }^{13} \mathrm{C} \mathrm{NMR}\left(125 \mathrm{MHz}, \mathrm{C}_{6} \mathrm{D}_{6}\right) \delta 2.4\left(\mathrm{C}_{1}\right)$; $7.0\left(\mathrm{C}_{6}\right) ; 8.1\left(\mathrm{C}_{12}\right) ; 37.2\left(\mathrm{C}_{11}\right) ; 37.7\left(\mathrm{C}_{5}\right) ; 44.1\left(\mathrm{C}_{7}\right) ; 72.5\left(\mathrm{C}_{4}\right) ; 77.1\left(\mathrm{C}_{8}\right) ; 80.2\left(\mathrm{C}_{2}\right) ; 83.6\left(\mathrm{C}_{3}\right)$; $92.4\left(\mathrm{C}_{13}\right) ; 114.1\left(\mathrm{C}_{10}\right) ; 126.8\left(\mathrm{C}_{17}\right) ; 128.2\left(\mathrm{C}_{15}\right) ; 128.4\left(\mathrm{C}_{16}\right) ; 140.3\left(\mathrm{C}_{14}\right) ; 141.4\left(\mathrm{C}_{9}\right)$; EIMS $m / z$ :

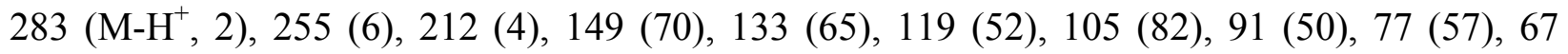
(100); EIHRMS $m / z$ : calcd for $\mathrm{C}_{19} \mathrm{H}_{23} \mathrm{O}_{2}, 283.16981$; found 283.16983; IR (NaCl, thin film) $\tilde{v}_{\max }$ $\left(\mathrm{cm}^{-1}\right) ; 921,1066,1124,1456,1652,2195,2970$. 


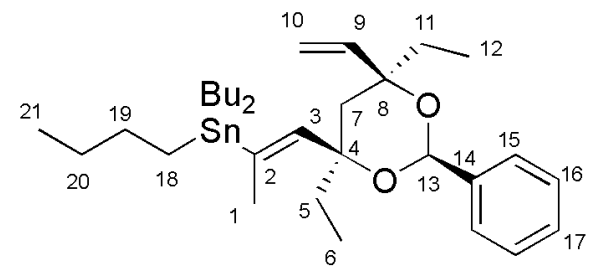

12

Preparation of $\quad(4 S, 6 R)$-Tributyl[2-(4,6-diethyl-2-phenyl-6-vinyl-1,3-dioxan-4-yl)-1methylvinyl]stannane 12: To a 10-mL, argon-filled, two-necked, round-bottomed flask equipped with a magnetic stirring bar was added palladium(II) acetate $(0.01 \mathrm{mmol}, 3 \mathrm{mg})$ and tricyclohexylphosphine $(0.03 \mathrm{mmol}, 7 \mathrm{mg})$ The flask was evacuated and filled with argon three times. Freshly distilled hexane $(3 \mathrm{~mL})$ was added and the solution stirred for 10 min until the solids had dissolved. 11 (70 $\mathrm{mg}, 0.25 \mathrm{mmol})$ in hexane was added dropwise via syringe. Tributyltin hydride $(0.49 \mathrm{mmol}, 144 \mathrm{mg}, 130 \mu \mathrm{L})$ was added slowly $(\sim 20 \mathrm{~min})$ via syringe. (NOTE: If hydrogen evolution was observed, the addition was paused until the bubbling stopped). The reaction was stirred for a further $30 \mathrm{~min}$, then immediately transferred to a silica gel column and rapidly eluted with hexane until the excess $\mathrm{Bu}_{3} \mathrm{SnH} /\left(\mathrm{Bu}_{3} \mathrm{Sn}\right)_{2}$ is removed, followed by elution with hexane/ether (10:1) to obtain a colorless oil which was a mixture of starting acetylene 11, product 12 and distannane 13. This mixture was subjected to further column chromatography $\left(\mathrm{SiO}_{2}\right.$ : hexanes/ether 500:1) to remove starting material, leaving an inseparable mixture of $\mathbf{1 2}$ and distannane 13. This mixture was used crude in the next reaction, but an analytical sample was obtained pure by careful chromatography, $\mathrm{R}_{\mathrm{f}} 0.80$ (hexanes/ether 19:1); ${ }^{1} \mathrm{H}$ NMR (300 MHz, $\left.\mathrm{C}_{6} \mathrm{D}_{6}\right) \delta$ 0.80-0.95 (m, 15H, $\left.\mathrm{H}_{6}, \mathrm{H}_{12}, \mathrm{H}_{21}\right) ; 1.30-1.76$ (m, 23H, $\mathrm{H}_{5}$, $\left.\mathrm{H}_{11}, \mathrm{H}_{7}, \mathrm{H}_{18}, \mathrm{H}_{19}, \mathrm{H}_{20}\right) 1.95$ (dq, J = 7.5, $13.0 \mathrm{~Hz}, 1 \mathrm{H}, \mathrm{H}_{5}$ or $\left.\mathrm{H}_{11}\right) ; 2.18$ (d, J = $1.8 \mathrm{~Hz}, 3 \mathrm{H}, \mathrm{H}_{1}$ ); $5.26\left(\mathrm{dd}, 1.8,11.0 \mathrm{~Hz}, 1 \mathrm{H}, \mathrm{H}_{10}\right) ; 5.40\left(\mathrm{dd}, \mathrm{J}=1.8,17.5 \mathrm{~Hz}, 1 \mathrm{H}, \mathrm{H}_{10}\right) ; 5.57$ (q, J = 2.1 Hz, 1H, $\left.\mathrm{H}_{3}\right) ; 5.79\left(\mathrm{dd}, \mathrm{J}=11.0,17.5 \mathrm{~Hz}, 1 \mathrm{H}, \mathrm{H}_{9}\right) ; 6.19\left(\mathrm{~s}, 1 \mathrm{H}, \mathrm{H}_{13}\right) ; 7.12-7.26\left(\mathrm{~m}, 3 \mathrm{H}, \mathrm{H}_{16}, \mathrm{H}_{17}\right) ; 7.81-$

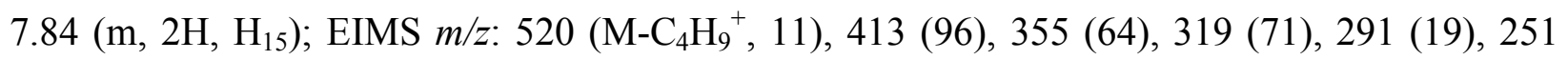
(32), 235 (44), 217 (36), 179 (100), 161 (93), 121 (54), 105 (99), 77 (50); EIHRMS m/z: calcd for $\mathrm{C}_{27} \mathrm{H}_{44} \mathrm{O}_{2} \mathrm{Sn}\left(\mathrm{M}-\mathrm{C}_{4} \mathrm{H}_{9}{ }^{+}\right), 520.23633$; found 520.23507. 


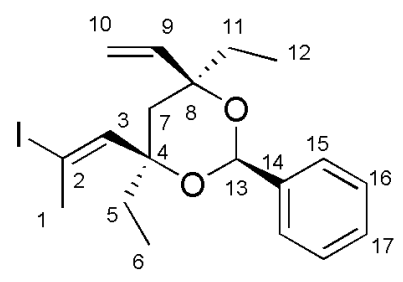

14

Preparation of (4S,6R)-4,6-Diethyl-4-(2-iodopropenyl)-2-phenyl-6-vinyl-1,3-dioxane 14: To a 25-mL, three-necked, round-bottomed flask equipped with a magnetic stirring bar was added $\mathrm{N}$-iodosuccinimide $(83 \mathrm{mg}, 0.37 \mathrm{mmol})$. The flask was placed under an argon atmosphere, and THF $\left(5 \mathrm{~mL}\right.$ ) was added via syringe. The mixture was cooled to $0{ }^{\circ} \mathrm{C}$ and crude 12 (from previous preparation, $0.25 \mathrm{mmol})$ in THF $(2 \mathrm{~mL})$ was added dropwise via syringe. The mixture was stirred at $0{ }^{\circ} \mathrm{C}$ for $2 \mathrm{~h}$, then poured into a separatory funnel containing saturated aqueous sodium thiosulfate $(3 \mathrm{~mL})$ and ether $(30 \mathrm{~mL})$. The funnel was shaken vigorously until the solution turned colorless, and the layers separated. The aqueous layer was extracted with ether $(3 \times 10 \mathrm{~mL})$ and the combined organic extracts washed with saturated brine $(20 \mathrm{~mL})$ and water $(20 \mathrm{~mL})$, dried over $\mathrm{MgSO}_{4}$, filtered and the solvent removed by rotary evaporation. The crude oil was purified by column chromatography ( $\mathrm{SiO}_{2}$ : hexanes) to yield 14 (50 mg, 49\% over 2 steps) as a colorless oil, $\mathrm{R}_{\mathrm{f}} 0.8$ (hexanes/ether 19:1); ${ }^{1} \mathrm{H}$ NMR $\left(500 \mathrm{MHz}, \mathrm{C}_{6} \mathrm{D}_{6}\right) \delta 0.82$ (t, J = 7.5 Hz, 3H, $\left.\mathrm{H}_{12}\right) ; 0.83$ $\left(\mathrm{t}, \mathrm{J}=7.5 \mathrm{~Hz}, 3 \mathrm{H}, \mathrm{H}_{6}\right) ; 1.26\left(\mathrm{~d}, \mathrm{~J}=13.2 \mathrm{~Hz}, 1 \mathrm{H}, \mathrm{H}_{7}\right) ; 1.44\left(\mathrm{~d}, \mathrm{~J}=13.2 \mathrm{~Hz}, 1 \mathrm{H}_{1} \mathrm{H}_{7}\right) ; 1.38-1.68(\mathrm{~m}$, $\left.4 \mathrm{H}, \mathrm{H}_{5}, \mathrm{H}_{11}\right) ; 2.44\left(\mathrm{~d}, \mathrm{~J}=1 \mathrm{~Hz}, 3 \mathrm{H}, \mathrm{H}_{1}\right) ; 5.18\left(\mathrm{dd}, \mathrm{J}=1.5,17.5 \mathrm{~Hz}, 1 \mathrm{H}, \mathrm{H}_{10}\right) ; 5.28(\mathrm{dd}, \mathrm{J}=1.5$, $\left.11.0 \mathrm{~Hz}, 1 \mathrm{H}, \mathrm{H}_{10}\right) ; 5.46\left(\mathrm{dd}, \mathrm{J}=11.0,17.5 \mathrm{~Hz}, 1 \mathrm{H}, \mathrm{H}_{9}\right) ; 5.95$ (s, 1H, H $\left.\mathrm{H}_{14}\right) ; 5.97$ (q, J = $1.0 \mathrm{~Hz}$, $\left.1 \mathrm{H}, \mathrm{H}_{3}\right) ; 7.10-7.22\left(\mathrm{~m}, 3 \mathrm{H}, \mathrm{H}_{16}, \mathrm{H}_{17}\right) ; 7.58-7.60\left(\mathrm{~d}, \mathrm{~J}=7.5 \mathrm{~Hz}, 2 \mathrm{H}, \mathrm{H}_{15}\right) ;{ }^{13} \mathrm{C} \mathrm{NMR}(125 \mathrm{MHz}$, $\left.\mathrm{C}_{6} \mathrm{D}_{6}\right) \delta 7.4\left(\mathrm{C}_{6}\right) ; 8.1\left(\mathrm{C}_{12}\right) ; 30.4\left(\mathrm{C}_{1}\right) ; 36.8\left(\mathrm{C}_{5}\right) ; 37.9\left(\mathrm{C}_{11}\right) ; 43.7\left(\mathrm{C}_{7}\right) ; 77.4\left(\mathrm{C}_{4}\right) ; 80.4\left(\mathrm{C}_{8}\right) ; 92.2$ $\left(\mathrm{C}_{2}\right)$; $98.9\left(\mathrm{C}_{14}\right) ; 115.5\left(\mathrm{C}_{10}\right) ; 127.3\left(\mathrm{C}_{15}\right) ; 128.7\left(\mathrm{C}_{17}\right) ; 129.1\left(\mathrm{C}_{16}\right) ; 140.0\left(\mathrm{C}_{3}\right) ; 140.5\left(\mathrm{C}_{14}\right) ; 143.7$ (C); EIMS m/z: 412 (M+1), 411 (3), 383 (6), 301 (9), 278 (49), 277 (73), 196 (63), 195 (81), 167 (60), 163 (76), 149 (79), 121 (74), 107 (92), 105 (100), 82 (68), 67 (74); EIHRMS m/z: calcd for $\mathrm{C}_{19} \mathrm{H}_{24} \mathrm{O}_{2} \mathrm{I}\left(\mathrm{M}-\mathrm{H}^{+}\right), 411.08211$; found 411.08267; IR $\left(\mathrm{NaCl}\right.$, thin film) $\tilde{v}_{\max }\left(\mathrm{cm}^{-1}\right) ; 921,1066$, $1122,1458,1645,2972$. 


\section{Synthesis of Sidechains}

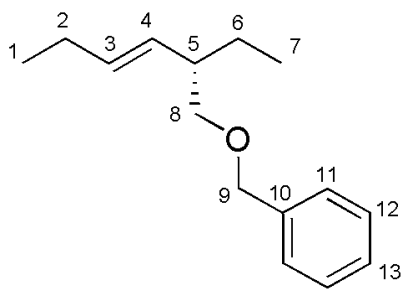

18

Preparation of $(\boldsymbol{S})$-(2-Ethylhex-3-enyloxymethyl)benzene 18: To a 500-mL, three-necked, round-bottomed flask equipped with a magnetic stirring bar was added 1-phenyl-5-(propane-1sulfonyl)-1H-tetrazole $17^{2}$ (1.46 g, $\left.5.79 \mathrm{mmol}\right)$. The flask was placed under an argon atmosphere, and THF $(250 \mathrm{~mL})$ was added via syringe. The mixture was cooled to $-78{ }^{\circ} \mathrm{C}$ and potassium hexamethyldisilazide $(6.37 \mathrm{mmol}, 0.5 \mathrm{M}$ solution in toluene, $12.74 \mathrm{~mL})$ was added via syringe. After $1 \mathrm{~h}$ of stirring at $-78^{\circ} \mathrm{C}, \mathbf{1 6}^{3}(1.10 \mathrm{~g}, 5.79 \mathrm{mmol})$ in THF $(20 \mathrm{~mL})$ was added dropwise via syringe over a period of $10 \mathrm{~min}$. The solution was allowed to warm slowly to $23{ }^{\circ} \mathrm{C}$ over a period of $2 \mathrm{~h}$ and then stirred for $16 \mathrm{~h}$. Water $(5 \mathrm{~mL})$ was added and the mixture stirred for a further $1 \mathrm{~h}$ before transfer to a separatory flask and dilution with ether $(500 \mathrm{~mL})$. Water $(100$ $\mathrm{mL})$ was added and the layers separated. The aqueous layer extracted with ether $(3 \times 100 \mathrm{~mL})$ and the combined organic extracts washed with saturated aqueous sodium bicarbonate $(100 \mathrm{~mL})$ and saturated brine $(100 \mathrm{~mL})$, dried over $\mathrm{MgSO}_{4}$, filtered and the solvent removed by rotary evaporation. The crude product was purified by column chromatography $\left(\mathrm{SiO}_{2}\right.$ : hexanes/ether $50: 1)$ to yield $18(720 \mathrm{mg}, 58 \%)$ as a colorless oil, $\mathrm{R}_{\mathrm{f}} 0.7$ (hexanes/ether 19:1); ${ }^{1} \mathrm{H}$ NMR (400 $\left.\mathrm{MHz} \mathrm{CDCl}_{3}\right) \delta 0.88\left(\mathrm{t}, \mathrm{J}=7.5 \mathrm{~Hz}, 3 \mathrm{H}_{1} \mathrm{H}_{7}\right) ; 1.00\left(\mathrm{t}, \mathrm{J}=7.5 \mathrm{~Hz}, 3 \mathrm{H}, \mathrm{H}_{1}\right) ; 1.16-1.32\left(\mathrm{~m}, 1 \mathrm{H}, \mathrm{H}_{6}\right)$; 1.51-1.66 (m, 1H, $\left.\mathrm{H}_{6}\right) ; 2.05\left(\mathrm{dqn}, \mathrm{J}=1.2,7.5 \mathrm{~Hz}, 2 \mathrm{H}, \mathrm{H}_{2}\right) ; 2.22\left(\mathrm{~m}, 1 \mathrm{H}, \mathrm{H}_{5}\right) ; 3.37(\mathrm{~d}, 6.3 \mathrm{~Hz}, 1 \mathrm{H}$, $\left.\mathrm{H}_{8}\right) ; 3.38\left(\mathrm{~d}, 6.3 \mathrm{~Hz}, 1 \mathrm{H}, \mathrm{H}_{8}\right) ; 4.53\left(\mathrm{~s}, 2 \mathrm{H}, \mathrm{H}_{9}\right) ; 5.23$ (ddt, J = 1.2, 8.1, 15.3, 1H, $\left.\mathrm{H}_{4}\right) ; 5.54$ (ddt, J $\left.=0.9,7.5,15.3 \mathrm{~Hz}, 1 \mathrm{H}, \mathrm{H}_{3}\right) ; 7.22-7.40\left(\mathrm{~m}, 5 \mathrm{H}, \mathrm{H}_{11-13}\right) ;{ }^{13} \mathrm{C} \mathrm{NMR}\left(75 \mathrm{MHz}, \mathrm{CDCl}_{3}\right) \delta 11.7\left(\mathrm{C}_{7}\right)$; $14.2\left(\mathrm{C}_{1}\right) ; 24.8\left(\mathrm{C}_{6}\right) ; 25.9\left(\mathrm{C}_{2}\right) ; 44.7\left(\mathrm{C}_{5}\right) ; 73.1\left(\mathrm{C}_{8}\right) ; 74.3\left(\mathrm{C}_{9}\right) ; 127.6\left(\mathrm{C}_{3}\right) ; 127.7\left(\mathrm{C}_{11}\right.$ or $\left.\mathrm{C}_{13}\right)$; $128.5\left(\mathrm{C}_{11}\right.$ or $\left.\mathrm{C}_{13}\right) ; 130.4\left(\mathrm{C}_{12}\right) ; 133.7\left(\mathrm{C}_{4}\right) ; 139.0\left(\mathrm{C}_{10}\right)$; IR $\left(\mathrm{NaCl}\right.$, thin film) $\tilde{v}_{\max }\left(\mathrm{cm}^{-1}\right) ; 968$, $1028,1362,1558,1652,2873,2931,3031$. 


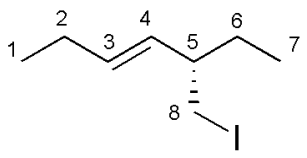

19

Preparation of $(\boldsymbol{S})$-5-Iodomethylhept-3-ene 19: A three-necked, 100-mL round-bottomed flask was equipped with a magnetic stirring bar and attached to a vacuum line, an oil bubbler and a tank of anhydrous ammonia. The flask was placed under vacuum, filled with argon and cooled to $-78^{\circ} \mathrm{C}$. The argon flow was stopped and ammonia was passed through the flask at $-78{ }^{\circ} \mathrm{C}$ until approximately $15 \mathrm{~mL}$ had condensed. The ammonia flow was stopped, the tank and bubbler disconnected and the flask placed under argon. Sodium $(6.19 \mathrm{mmol}, 142 \mathrm{mg})$ was cut into $1 \mathrm{~mm}^{3}$ pieces, washed quickly with anhydrous ether to remove any residual oil, and added in portions to the flask at $-78^{\circ} \mathrm{C}$. The liquid turned a deep blue color and was stirred at $-78{ }^{\circ} \mathrm{C}$ for 30 min until all of the sodium had dissolved. Ether $18(1.24 \mathrm{mmol}, 270 \mathrm{mg})$ in THF (10 mL) was added via syringe and the mixture stirred for $15 \mathrm{~min}$ at $-78{ }^{\circ} \mathrm{C}$ (blue color persists). $\mathrm{NH}_{4} \mathrm{Cl}$ (12.38 $\mathrm{mmol}$, $662 \mathrm{mg}$ ) was added and the solution stirred until the blue color disappeared. $\mathrm{MeOH}(5 \mathrm{~mL})$ was added and the flask opened to air and allowed to warm to $23{ }^{\circ} \mathrm{C}$, then stirred for $2 \mathrm{~h}$ to allow the ammonia to evaporate. Water $(10 \mathrm{~mL})$ and ether $(50 \mathrm{~mL})$ were added and the mixture was transferred to a separatory flask. The layers were separated and the aqueous layer extracted with ether $(3 \times 20 \mathrm{~mL})$. The combined organic extracts were washed with water $(10 \mathrm{~mL})$ and saturated brine $(20 \mathrm{~mL})$, dried over $\mathrm{MgSO}_{4}$, filtered and the solvent removed by rotary evaporation. The crude product $(S)$-2-ethylhex-3-en-1-ol was not purified due to the small quantity and the molecule's volatility, but used crude in the next reaction. $\mathrm{R}_{\mathrm{f}} 0.25$ (hexanes/ether $4: 1$ ).

To a 50-mL, three-necked, round-bottomed flask equipped with a magnetic stirring bar and 10-mL pressure-equalizing dropping funnel was added imidazole (236 mg, $3.46 \mathrm{mmol}$ ) and triphenylphosphine (907 mg, $3.46 \mathrm{mmol})$. The flask was placed under an argon atmosphere, and ether $(6 \mathrm{~mL})$ was added via syringe. The mixture was cooled to $0{ }^{\circ} \mathrm{C}$ and a solution of iodine $(3.46 \mathrm{mmol}, 878 \mathrm{mg})$ in acetonitrile $(3 \mathrm{~mL})$ was added via dropping funnel over a period of 30 min (NOTE: if yellow color develops, slow the addition of iodine). Crude $(S)$-2-ethylhex-3-en-1ol $(150 \mathrm{mg}, 1.15 \mathrm{mmol})$ in ether $(3 \mathrm{~mL})$ was added via syringe and the solution was allowed to warm slowly to $23{ }^{\circ} \mathrm{C}$ over a period of $2 \mathrm{~h}$, then saturated aqueous sodium thiosulfate $(5 \mathrm{~mL})$ was added. The mixture was transferred to a separatory flask and diluted with hexanes (50 mL). 
Water $(10 \mathrm{~mL})$ was added and the layers separated. The aqueous layer extracted with hexanes (3 x $20 \mathrm{~mL}$ ) and the combined organic extracts washed with saturated aqueous sodium bicarbonate $(20 \mathrm{~mL})$ and saturated brine $(20 \mathrm{~mL})$, dried over $\mathrm{MgSO}_{4}$, filtered and the solvent removed by rotary evaporation. Hexanes $(30 \mathrm{~mL})$ was added to the resulting mixture of crude product and triphenylphosphine oxide, and the mixture filtered through a Celite pad, and the solvent removed by rotary evaporation. This was repeated if any triphenylphosphine oxide remained. The crude product was purified by column chromatography $\left(\mathrm{SiO}_{2}\right.$ : hexanes) to yield $19(131 \mathrm{mg}, 63 \%)$ as a colorless oil, $\mathrm{R}_{\mathrm{f}} 0.7$ (hexane); ${ }^{1} \mathrm{H}$ NMR $\left(500 \mathrm{MHz}, \mathrm{C}_{6} \mathrm{D}_{6}\right) \delta 0.69$ (t, J = $\left.7.5 \mathrm{~Hz}, 3 \mathrm{H}, \mathrm{H}_{1}\right) ; 0.89$ (t, J $\left.=7.5 \mathrm{~Hz}, 3 \mathrm{H}, \mathrm{H}_{7}\right) ; 1.05-1.12\left(\mathrm{~m}, 1 \mathrm{H}, \mathrm{H}_{6}\right) ; 1.30-1.38\left(\mathrm{~m}, 1 \mathrm{H}, \mathrm{H}_{6}\right) ; 1.68-1.72\left(\mathrm{~m}, 1 \mathrm{H}, \mathrm{H}_{5}\right) ; 1.88$ (dqn, $\left.\mathrm{J}=1.5,7.5 \mathrm{~Hz}, 2 \mathrm{H}, \mathrm{H}_{2}\right) ; 2.80\left(\mathrm{~d}, \mathrm{~J}=6.0 \mathrm{~Hz}, 2 \mathrm{H}, \mathrm{H}_{8}\right) ; 4.96(\mathrm{ddt}, \mathrm{J}=1.5,8.5,16.5 \mathrm{~Hz}, 1 \mathrm{H}$, $\left.\mathrm{H}_{4}\right) ; 5.30\left(\mathrm{ddt}, \mathrm{J}=1,6.5,16.5 \mathrm{~Hz}, 1 \mathrm{H}, \mathrm{H}_{3}\right) ;{ }^{13} \mathrm{C} \mathrm{NMR}\left(125 \mathrm{MHz}, \mathrm{C}_{6} \mathrm{D}_{6}\right) \delta 11.4\left(\mathrm{C}_{7}\right) ; 13.9\left(\mathrm{C}_{1}\right)$; $25.8\left(\mathrm{C}_{6}\right) ; 27.9\left(\mathrm{C}_{2}\right) ; 31.8\left(\mathrm{C}_{5}\right) ; 45.9\left(\mathrm{C}_{8}\right) ; 131.1\left(\mathrm{C}_{3}\right) ; 134.3\left(\mathrm{C}_{4}\right)$; EIMS m/z: $240\left(\mathrm{M}^{+}, 5\right), 183$ (17), 151 (48), 123 (55), 105 (79), 84 (100); EIHRMS m/z: calcd for $\mathrm{C}_{8} \mathrm{H}_{15} \mathrm{I}, 238.02201$; found 238.02141 .

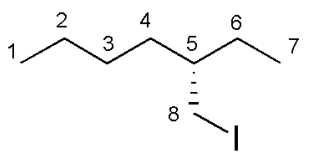

20

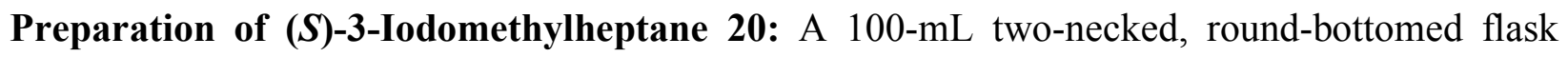
equipped with magnetic stirring bar, vacuum inlet and three-way stopcock attached to a balloon and a hydrogen tank was charged with palladium on carbon $(10 \% \mathrm{Pd}, 0.139 \mathrm{mmol} \mathrm{Pd}, 150 \mathrm{mg})$, 18 (300 mg, $1.39 \mathrm{mmol})$ and methanol $(10 \mathrm{~mL})$. The flask was placed under vacuum until the solvent began to boil, at which point the flask was filled with hydrogen gas from the balloon. This procedure was repeated 10 times, and the mixture was stirred for $2 \mathrm{~h}$. The balloon was removed and the mixture filtered through a Celite pad, washing the pad with ethyl acetate. The solvent was removed by rotary evaporation. The crude product $(S)$-2-ethylhexan-1-ol was not purified due to the small quantity and the molecule's volatility, but used crude in the next reaction. $\mathrm{R}_{\mathrm{f}} 0.25$ (hexanes/ether $4: 1$ ).

Crude (S)-2-ethylhexan-1-ol was converted to $\mathbf{2 0}$ on a $0.8 \mathrm{mmol}$ scale using the procedure previously reported for the synthesis of $\mathbf{1 9}$, yielding $20(83 \%)$ as a colorless oil after 
column chromatography ( $\mathrm{SiO}_{2}$; hexanes), $\mathrm{R}_{\mathrm{f}} 0.75$ (hexanes); ${ }^{1} \mathrm{H}$ NMR $\left(300 \mathrm{MHz}, \mathrm{CDCl}_{3}\right) \delta 0.88$ (t, $\mathrm{J}=7.5 \mathrm{~Hz}, 3 \mathrm{H}, \mathrm{H}_{1}$ or $\left.\mathrm{H}_{7}\right) ; 0.91$ (t, $\mathrm{J}=7.5 \mathrm{~Hz}, 3 \mathrm{H}, \mathrm{H}_{1}$ or $\left.\mathrm{H}_{7}\right) ; 1.20-1.45$ (m, 9Hz, $\mathrm{H}_{2}, \mathrm{H}_{3}, \mathrm{H}_{4}$, $\left.\mathrm{H}_{5}, \mathrm{H}_{6}\right) ; 3.27\left(\mathrm{dd}, \mathrm{J}=9.9,14.4 \mathrm{~Hz}, 1 \mathrm{H}, \mathrm{H}_{8}\right) ; 3.28\left(\mathrm{dd}, \mathrm{J}=9.9,14.4 \mathrm{~Hz}, 1 \mathrm{H}, \mathrm{H}_{8}\right) ;{ }^{13} \mathrm{C}$ NMR $(75$ $\left.\mathrm{MHz}, \mathrm{CDCl}_{3}\right) \delta 11.1\left(\mathrm{C}_{1}\right) ; 14.3\left(\mathrm{C}_{7}\right) ; 16.4\left(\mathrm{C}_{2}\right) ; 23.0\left(\mathrm{C}_{3}\right) ; 27.4\left(\mathrm{C}_{4}\right) ; 29.0\left(\mathrm{C}_{6}\right) ; 33.9\left(\mathrm{C}_{5}\right) ; 40.5$ $\left(\mathrm{C}_{8}\right)$; EIMS m/z: $238\left(\mathrm{M}^{+}, 7\right), 209$ (15), 170 (58), 141 (76), 111 (92), 84 (100); EIHRMS m/z: calcd for $\mathrm{C}_{8} \mathrm{H}_{17} \mathrm{I}, 240.0375$; found 240.0363 .

\section{Synthesis of Plakortone B}

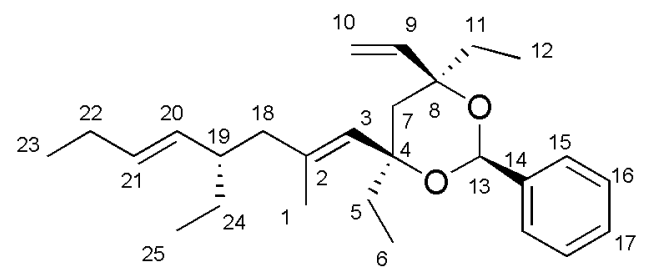

22

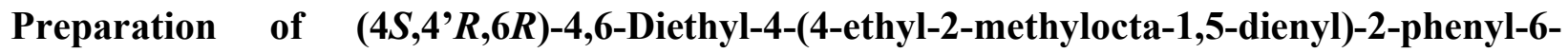
vinyl-1,3-dioxane 22: A $25-\mathrm{mL}$ three-necked, oven-dried, round-bottomed flask was placed under an argon atmosphere. Iodide $19(37 \mathrm{mg}, 0.16 \mathrm{mmol})$ in ether $(5 \mathrm{~mL})$ was added via syringe, and the solution cooled to $-78^{\circ} \mathrm{C}$. tert-BuLi $(0.31 \mathrm{mmol}, 1.70 \mathrm{M}$ in pentane, $270 \mu \mathrm{L})$ was added rapidly via syringe and the solution stirred for $3 \mathrm{~min}$. Next, 9-methoxy-9borabicyclo[3.3.1.]nonane $(0.513 \mathrm{mmol}, 1 \mathrm{M}$ in THF, $513 \mu \mathrm{L})$ was added via syringe followed by THF $(5 \mathrm{~mL})$. The solution was stirred for $10 \mathrm{~min}$ at $-78{ }^{\circ} \mathrm{C}$, and then warmed slowly to $23^{\circ} \mathrm{C}$, then stirred at that temperature for $1 \mathrm{~h}$. Aqueous $\mathrm{K}_{3} \mathrm{PO}_{4}(0.513 \mathrm{mmol}, 3 \mathrm{M}$ solution, $171 \mu \mathrm{L})$ was added followed by vinyl iodide $14(0.117 \mathrm{mmol}, 48 \mathrm{mg})$ in THF $(1 \mathrm{~mL})$, DMF $(5 \mathrm{~mL})$ and [1, 'bis(diphenylphosphino)-ferrocene]dichloropalladium(II) complex with $\mathrm{CHCl}_{3}(0.012 \mathrm{mmol}, 10$ $\mathrm{mg})$. The resulting black solution was stirred at $23{ }^{\circ} \mathrm{C}$ for $16 \mathrm{~h}$. $\mathrm{Et}_{2} \mathrm{O}(10 \mathrm{~mL})$ was added and the mixture was transferred to a separatory flask. Water $(10 \mathrm{~mL})$ was added and the layers were separated and the aqueous layer extracted with ether $(3 \times 10 \mathrm{~mL})$. The combined organic extracts were washed with water $(5 \times 10 \mathrm{~mL})$ and saturated brine $(20 \mathrm{~mL})$, dried over $\mathrm{MgSO}_{4}$, filtered and the solvent removed by rotary evaporation. The crude product was purified by column chromatography $\left(\mathrm{SiO}_{2}\right.$ : hexanes/ether 500:1) to yield $22(42 \mathrm{mg}, 77 \%)$ as a colorless oil, $\mathrm{R}_{\mathrm{f}} 0.85$ 
(hexanes/ether 19:1); ${ }^{1} \mathrm{H}$ NMR (500 MHz, $\left.\mathrm{C}_{6} \mathrm{D}_{6}\right) \delta 0.93\left(\mathrm{t}, \mathrm{J}=7.5 \mathrm{~Hz}, 3 \mathrm{H}, \mathrm{H}_{23}\right.$ or $\mathrm{H}_{25}$ ); $0.94(\mathrm{t}, \mathrm{J}$ $=7.5 \mathrm{~Hz}, 3 \mathrm{H}, \mathrm{H}_{23}$ or $\left.\mathrm{H}_{25}\right) ; 0.95\left(\mathrm{t}, \mathrm{J}=7.5 \mathrm{~Hz}, 3 \mathrm{H}, \mathrm{H}_{6}\right.$ or $\left.\mathrm{H}_{12}\right) ; 0.96\left(\mathrm{t}, \mathrm{J}=7.5 \mathrm{~Hz}, 3 \mathrm{H}, \mathrm{H}_{6}\right.$ or $\left.\mathrm{H}_{12}\right)$; $1.19\left(\mathrm{dq}, \mathrm{J}=7.5,13.0 \mathrm{~Hz}, 2 \mathrm{H}, \mathrm{H}_{24}\right) ; 1.55\left(\mathrm{dq}, \mathrm{J}=7.5,13.0 \mathrm{~Hz}, 1 \mathrm{H}, \mathrm{H}_{5}\right.$ or $\left.\mathrm{H}_{11}\right) ; 1.58(\mathrm{~d}, \mathrm{~J}=13.5$ $\left.\mathrm{Hz}, \mathrm{H}_{7}\right) ; 1.68\left(\mathrm{dq}, \mathrm{J}=7.5,13.0 \mathrm{~Hz}, 1 \mathrm{H}, \mathrm{H}_{5}\right.$ or $\left.\mathrm{H}_{11}\right) ; 1.70\left(\mathrm{~d}, \mathrm{~J}=13.5 \mathrm{~Hz}, \mathrm{H}_{7}\right) ; 1.75(\mathrm{dq}, \mathrm{J}=7.5$, $13.0 \mathrm{~Hz}, 1 \mathrm{H}, \mathrm{H}_{5}$ or $\left.\mathrm{H}_{11}\right) ; 1.79\left(\mathrm{~d}, \mathrm{~J}=1.5 \mathrm{~Hz}, 3 \mathrm{H}, \mathrm{H}_{1}\right) ; 1.87-2.12\left(\mathrm{~m}, 6 \mathrm{H}, \mathrm{H}_{18}, \mathrm{H}_{19}, \mathrm{H}_{22}, \mathrm{H}_{11}\right) ; 5.13$ (q, J = 1.5 Hz, 1H, $\left.\mathrm{H}_{3}\right) ; 5.16\left(\mathrm{ddt}, \mathrm{J}=1.5,7.0,15 \mathrm{~Hz}, 1 \mathrm{H}, \mathrm{H}_{20}\right) ; 5.20$ (dd, 2.0, $\left.11.0 \mathrm{~Hz}, 1 \mathrm{H}, \mathrm{H}_{10}\right)$; $5.27\left(\mathrm{dd}, \mathrm{J}=2.0,17.5 \mathrm{~Hz}, 1 \mathrm{H}, \mathrm{H}_{10}\right) ; 5.36\left(\mathrm{dt}, \mathrm{J}=6.5,15.0 \mathrm{~Hz}, 1 \mathrm{H}, \mathrm{H}_{21}\right) ; 5.72(\mathrm{dd}, \mathrm{J}=11.0,17.5$ $\left.\mathrm{Hz}, 1 \mathrm{H}, \mathrm{H}_{9}\right) ; 6.06$ (s, $\left.1 \mathrm{H}, \mathrm{H}_{13}\right) ; 7.12-7.26\left(\mathrm{~m}, 3 \mathrm{H}, \mathrm{H}_{16}, \mathrm{H}_{17}\right) ; 7.81$ (dd, J = 1.0, $\left.8.5 \mathrm{~Hz}, \mathrm{H}_{15}\right) ;{ }^{13} \mathrm{C}$ NMR (125 MHz, $\left.\mathrm{C}_{6} \mathrm{D}_{6}\right) \delta$ 7.6 $\left(\mathrm{C}_{12}\right) ; 8.6\left(\mathrm{C}_{6}\right) ; 12.3\left(\mathrm{C}_{25}\right) ; 14.8\left(\mathrm{C}_{23}\right) ; 17.8\left(\mathrm{C}_{1}\right) ; 26.5\left(\mathrm{C}_{22}\right) ; 28.7$ $\left(\mathrm{C}_{24}\right) ; 37.2\left(\mathrm{C}_{11}\right) ; 38.3\left(\mathrm{C}_{5}\right) ; 43.5\left(\mathrm{C}_{19}\right) ; 44.4\left(\mathrm{C}_{18}\right) ; 47.8\left(\mathrm{C}_{7}\right) ; 77.3\left(\mathrm{C}_{4}\right) ; 78.0\left(\mathrm{C}_{8}\right) ; 91.7\left(\mathrm{C}_{13}\right)$; $114.8\left(\mathrm{C}_{10}\right) ; 127.4\left(\mathrm{C}_{3}\right) ; 127.9\left(\mathrm{C}_{17}\right) ; 128.8\left(\mathrm{C}_{16}\right) ; 128.9\left(\mathrm{C}_{15}\right) ; 132.8\left(\mathrm{C}_{21}\right) ; 133.8\left(\mathrm{C}_{20}\right) ; 138.4$

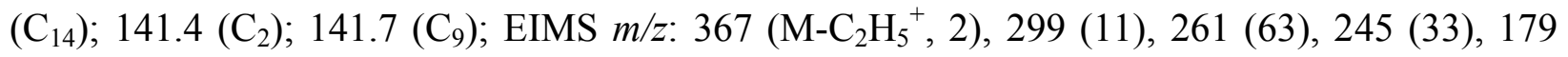
(82), 163 (66), 151 (73), 135 (65), 121 (68), 105 (93), 82 (100); EIHRMS m/z: calcd for

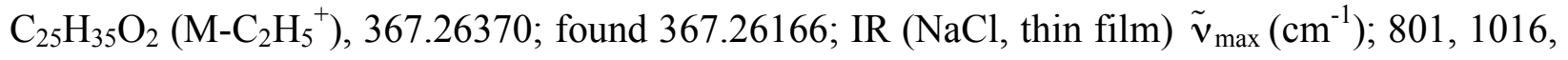
1260, 1457, 2927.

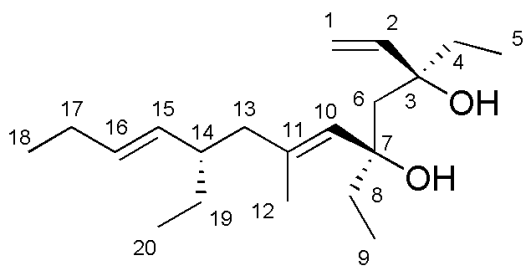

23

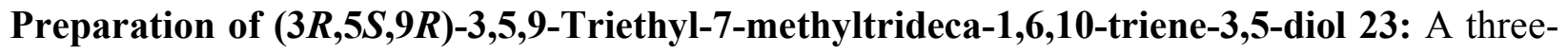
necked, 50-mL round-bottomed flask was equipped with a magnetic stirring bar and attached to a vacuum line, an oil bubbler and a tank of anhydrous ammonia. The flask was placed under vacuum, filled with argon and cooled to $-78^{\circ} \mathrm{C}$. The argon flow was ceased and ammonia was passed through the flask at $-78{ }^{\circ} \mathrm{C}$ until approximately $5 \mathrm{~mL}$ had condensed. The ammonia flow was ceased, the tank and bubbler disconnected and the flask placed under argon. Sodium (0.88 mmol, $20 \mathrm{mg}$ ) was cut into $1 \mathrm{~mm}^{3}$ pieces, washed quickly with anhydrous ether to remove any residual oil, and added in portions to the flask at $-78^{\circ} \mathrm{C}$. The liquid turned a deep blue color and 
was stirred at $-78{ }^{\circ} \mathrm{C}$ for 30 min until all of the sodium had dissolved. Isopropanol $(200 \mu \mathrm{L})$ was added via syringe, followed by $22(0.088 \mathrm{mmol}, 35 \mathrm{mg})$ in THF $(5 \mathrm{~mL})$, and the mixture stirred for $15 \mathrm{~min}$ at $-78{ }^{\circ} \mathrm{C}$ (blue color persists). $\mathrm{NH}_{4} \mathrm{Cl}(1.72 \mathrm{mmol}, 92 \mathrm{mg}$ ) was added and the solution stirred until the blue color disappeared. $\mathrm{MeOH}(2 \mathrm{~mL})$ was added and the flask opened to air and allowed to warm to $23{ }^{\circ} \mathrm{C}$, then stirred for $2 \mathrm{~h}$ to allow the ammonia to evaporate. Water $(5 \mathrm{~mL})$ and ether $(20 \mathrm{~mL})$ were added and the mixture was transferred to a separatory flask. The layers were separated and the aqueous layer extracted with ether $(3 \times 10 \mathrm{~mL})$. The combined organic extracts were washed with water $(10 \mathrm{~mL})$ and saturated brine $(20 \mathrm{~mL})$, dried over $\mathrm{MgSO}_{4}$, filtered and the solvent removed by rotary evaporation. The crude product was purified by column chromatography $\left(\mathrm{SiO}_{2}\right.$ : hexanes/ether 19:1) to yield $23(17 \mathrm{mg}, 65 \%)$ as a colorless oil, $\mathrm{R}_{\mathrm{f}} 0.25$ (hexanes/ether 4:1); ${ }^{1} \mathrm{H}$ NMR $\left(500 \mathrm{MHz}, \mathrm{C}_{6} \mathrm{D}_{6}\right) \delta 0.90\left(\mathrm{t}, \mathrm{J}=7.5 \mathrm{~Hz}, 3 \mathrm{H}, \mathrm{H}_{18}\right.$ or $\left.\mathrm{H}_{20}\right)$; $0.92\left(\mathrm{t}, \mathrm{J}=7.5 \mathrm{~Hz}, 3 \mathrm{H}, \mathrm{H}_{18}\right.$ or $\left.\mathrm{H}_{20}\right) ; 0.93\left(\mathrm{t}, \mathrm{J}=7.5 \mathrm{~Hz}, 3 \mathrm{H}, \mathrm{H}_{5}\right.$ or $\left.\mathrm{H}_{9}\right) ; 0.99$ (t, J = 7.5 Hz, 3H, $\mathrm{H}_{5}$ or $\left.\mathrm{H}_{9}\right)$; 1.23-1.48 (m, 6H, $\left.\mathrm{H}_{4}, \mathrm{H}_{8}, \mathrm{H}_{19}\right) ; 1.41$ (dq, $\mathrm{J}=7.5,13.0 \mathrm{~Hz}, 1 \mathrm{H}, \mathrm{H}_{4}$ or $\left.\mathrm{H}_{8}\right) ; 1.52$ (dq, $\mathrm{J}=$ 7.5, $13.0 \mathrm{~Hz}, 1 \mathrm{H}, \mathrm{H}_{4}$ or $\left.\mathrm{H}_{8}\right) ; 1.63\left(\mathrm{dq}, \mathrm{J}=7.5,13.0 \mathrm{~Hz}, 1 \mathrm{H}, \mathrm{H}_{4}\right.$ or $\left.\mathrm{H}_{8}\right) ; 1.72\left(\mathrm{~d}, \mathrm{~J}=14.5 \mathrm{~Hz}, \mathrm{H}_{6}\right.$ ); $1.77\left(\mathrm{~d}, \mathrm{~J}=1.5 \mathrm{~Hz}, 3 \mathrm{H}, \mathrm{H}_{12}\right) ; 1.79$ (d, J = 14.5 Hz, H6); 1.92-2.08 (m, 5H, H $\left.\mathrm{H}_{13}, \mathrm{H}_{14}, \mathrm{H}_{17}\right) ; 2.65$ (br s, OH); 3.02 (br s, OH); $5.02\left(\mathrm{dd}, 2.0,10.5 \mathrm{~Hz}, 1 \mathrm{H}, \mathrm{H}_{1}\right) ; 5.03$ (q, J = $\left.1.5 \mathrm{~Hz}, 1 \mathrm{H}, \mathrm{H}_{10}\right) ; 5.15$ $\left(\mathrm{ddt}, \mathrm{J}=1.5,8.5,15.5 \mathrm{~Hz}, 1 \mathrm{H}, \mathrm{H}_{15}\right) ; 5.31\left(\mathrm{dd}, \mathrm{J}=2.0,17.5 \mathrm{~Hz}, 1 \mathrm{H}, \mathrm{H}_{1}\right) ; 5.42(\mathrm{dt}, \mathrm{J}=6.0,15.5$ $\left.\mathrm{Hz}, 1 \mathrm{H}, \mathrm{H}_{16}\right) ; 5.76\left(\mathrm{dd}, \mathrm{J}=10.5,17.0 \mathrm{~Hz}, 1 \mathrm{H}, \mathrm{H}_{2}\right) ;{ }^{13} \mathrm{C} \mathrm{NMR}\left(125 \mathrm{MHz}, \mathrm{C}_{6} \mathrm{D}_{6}\right) \delta 8.0\left(\mathrm{C}_{5}\right) ; 8.4$ $\left(\mathrm{C}_{9}\right) ; 12.3\left(\mathrm{C}_{20}\right) ; 15.9\left(\mathrm{C}_{18}\right) ; 26.5\left(\mathrm{C}_{12}\right) ; 28.4\left(\mathrm{C}_{17}\right) ; 30.6\left(\mathrm{C}_{19}\right) ; 36.8\left(\mathrm{C}_{4}\right) ; 37.6\left(\mathrm{C}_{8}\right) ; 43.3\left(\mathrm{C}_{14}\right)$; $48.3\left(\mathrm{C}_{13}\right) ; 51.3\left(\mathrm{C}_{6}\right) ; 76.6\left(\mathrm{C}_{7}\right) ; 77.8\left(\mathrm{C}_{3}\right) ; 111.7\left(\mathrm{C}_{1}\right) ; 132.6\left(\mathrm{C}_{10}\right) ; 133.2\left(\mathrm{C}_{15}\right) ; 134.0\left(\mathrm{C}_{16}\right)$; 134.6 $\left(\mathrm{C}_{11}\right) ; 145.5\left(\mathrm{C}_{2}\right)$; ESMS m/z: $359\left(\mathrm{M}+\mathrm{Na}^{+}\right)$.

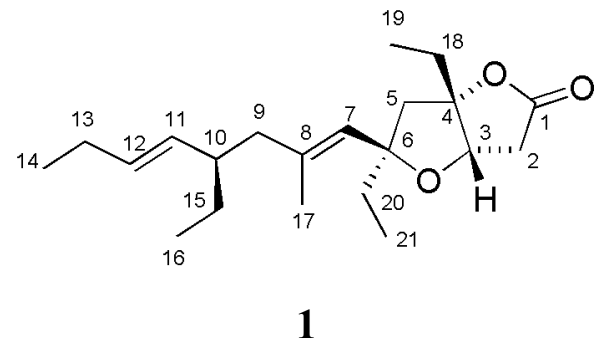

Preparation of Plakortone B 1: A 50-mL oven-dried, three-necked, round-bottomed flask was equipped with a magnetic stirring bar and septum and attached to a vacuum line and a three-way adapter connected to a carbon monoxide cylinder and balloon. Palladium chloride $(1 \mathrm{mg}, 0.007$ 
$\mathrm{mmol})$, anhydrous cupric chloride $(0.21 \mathrm{mmol}, 28 \mathrm{mg})$ and anhydrous sodium acetate $(0.21$ mmol, $18 \mathrm{mg}$ ) were added, and the flask placed under vacuum. The balloon was filled with carbon monoxide and the vacuum tap closed. The flask was filled with carbon monoxide from the balloon. The evacuation/fill procedure was repeated three times, and the flask was left under balloon pressure of carbon monoxide. Diol 23 (22 mg, $0.071 \mathrm{mmol})$ in glacial acetic acid (500 $\mu \mathrm{L}$ ) was added via syringe and the mixture stirred for $24 \mathrm{~h}$, at which point the color had gone from blue to pale brown. The balloon was removed and ether $(50 \mathrm{~mL})$ was added, then the mixture was transferred to a separatory funnel. Water $(10 \mathrm{~mL})$ was added, followed by slow addition of $2 \%$ aqueous $\mathrm{NaOH}(10 \mathrm{~mL})$. The layers were separated and the organic layer was washed with $2 \%$ aqueous $\mathrm{NaOH}(2 \times 10 \mathrm{~mL})$, saturated sodium bicarbonate $(10 \mathrm{~mL})$, saturated brine $(10 \mathrm{~mL})$, dried over $\mathrm{MgSO}_{4}$, filtered and the solvent removed by rotary evaporation. The resulting crude yellow oil was purified by column chromatography $\left(\mathrm{SiO}_{2}\right.$ : hexanes/ether 2:1) to yield $1(18 \mathrm{mg}, 75 \%)$ as a colorless oil, $\mathrm{R}_{\mathrm{f}} 0.2$ (hexanes/ether $\left.4: 1\right) ;{ }^{1} \mathrm{H} \mathrm{NMR}\left(500 \mathrm{MHz}, \mathrm{CDCl}_{3}\right) \delta$ $0.84\left(\mathrm{t}, \mathrm{J}=7.5 \mathrm{~Hz}, 3 \mathrm{H}, \mathrm{H}_{21}\right) ; 0.87\left(\mathrm{t}, \mathrm{J}=7.5 \mathrm{~Hz}, 3 \mathrm{H}, \mathrm{H}_{16}\right) ; 0.96$ (t, J = $\left.7.5 \mathrm{~Hz}, \mathrm{H}_{14}\right) ; 0.98$ (t, J = $\left.7.5 \mathrm{~Hz}, 3 \mathrm{H}, \mathrm{H}_{19}\right) ; 1.30-1.38\left(\mathrm{~m}, 2 \mathrm{H}, \mathrm{H}_{15}\right) ; 1.69$ (d, J = $\left.1.5 \mathrm{~Hz}, 3 \mathrm{H}, \mathrm{H}_{17}\right) ; 1.68-1.77$ (m, 4H, H18, $\mathrm{H}_{20}$ ); 1.88-1.92 (m, 2H, H $)$; 1.95 (d, J = 13.5 Hz, 1H, H $)$; 1.94-2.06 (m, 5H, H, $\left.\mathrm{H}_{10}, \mathrm{H}_{13}\right) ; 2.14$ $\left(\mathrm{d}, \mathrm{J}=13.5 \mathrm{~Hz}, 1 \mathrm{H}, \mathrm{H}_{5}\right) ; 2.25\left(\mathrm{~d}, \mathrm{~J}=13.5 \mathrm{~Hz}, 1 \mathrm{H}, \mathrm{H}_{5}\right) ; 2.65\left(\mathrm{dd}, \mathrm{J}=1.5,18.5 \mathrm{~Hz}, 1 \mathrm{H}, \mathrm{H}_{2}\right) ; 2.71$ $\left(\mathrm{dd}, \mathrm{J}=6.0,18.5 \mathrm{~Hz}, 1 \mathrm{H}, \mathrm{H}_{2}\right) ; 4.21\left(\mathrm{dd}, \mathrm{J}=1.5,5.0 \mathrm{~Hz}, 1 \mathrm{H}, \mathrm{H}_{3}\right) ; 5.03$ (ddt, J = 1.0, 8.5, 15.5, 1H, $\left.\mathrm{H}_{11}\right) ; 5.06\left(\mathrm{q}, \mathrm{J}=1.0 \mathrm{~Hz}, 1 \mathrm{H}, \mathrm{H}_{7}\right) ; 5.36\left(\mathrm{dt}, \mathrm{J}=6.5,15.5 \mathrm{~Hz}, 1 \mathrm{H}, \mathrm{H}_{12}\right) ;{ }^{13} \mathrm{C} \mathrm{NMR}(125 \mathrm{MHz}$, $\left.\mathrm{CDCl}_{3}\right) \delta 8.7\left(\mathrm{C}_{21}\right) ; 9.0\left(\mathrm{C}_{19}\right) ; 11.9\left(\mathrm{C}_{16}\right) ; 14.3\left(\mathrm{C}_{14}\right) ; 16.9\left(\mathrm{C}_{17}\right) ; 25.9\left(\mathrm{C}_{13}\right) ; 28.2\left(\mathrm{C}_{15}\right) ; 30.6\left(\mathrm{C}_{18}\right)$; $34.0\left(\mathrm{C}_{20}\right) ; 36.9\left(\mathrm{C}_{2}\right) ; 42.8\left(\mathrm{C}_{10}\right)$; $47.2\left(\mathrm{C}_{9}\right)$; $49.0\left(\mathrm{C}_{5}\right) ; 79.8\left(\mathrm{C}_{3}\right) ; 87.1\left(\mathrm{C}_{6}\right)$; $97.5\left(\mathrm{C}_{4}\right) ; 129.8\left(\mathrm{C}_{7}\right)$;

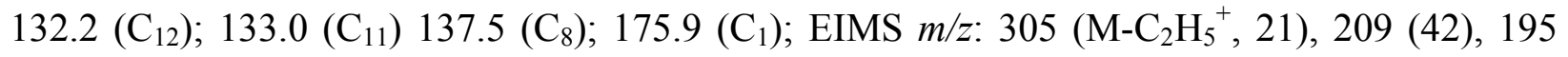
(11), 149 (14), 97 (100), 83 (39), 69 (52); EIHRMS m/z: calcd for $\mathrm{C}_{19} \mathrm{H}_{29} \mathrm{O}_{3}$, 305.21167; found 305.21148 , spectral data consistent with published spectra. ${ }^{4}$ 


\section{Synthesis of Dihydroplakortone B}

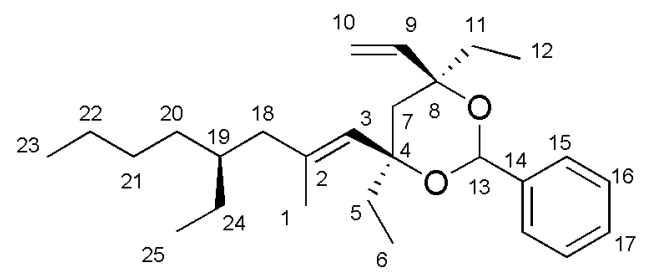

25

Preparation of $(4 S, 4 ' R, 6 R)-4,6$-Diethyl-4-(4-ethyl-2-methyloct-1-enyl)-2-phenyl-6-vinyl[1,3]dioxane 25: 14 was converted to 25 on a $0.1 \mathrm{mmol}$ scale using the procedure previously reported for the synthesis of 22, yielding 25 (81\%) as a colorless oil after column chromatography $\left(\mathrm{SiO}_{2}\right.$; hexanes/ether 500:1), $\mathrm{R}_{\mathrm{f}} 0.8$ (hexanes/ether 19:1); ${ }^{1} \mathrm{H} \mathrm{NMR}(500 \mathrm{MHz}$, $\left.\mathrm{C}_{6} \mathrm{D}_{6}\right) \delta 0.89\left(\mathrm{t}, \mathrm{J}=7.5 \mathrm{~Hz}, 3 \mathrm{H}, \mathrm{H}_{23}\right.$ or $\left.\mathrm{H}_{25}\right) ; 0.90\left(\mathrm{t}, \mathrm{J}=7.5 \mathrm{~Hz}, 3 \mathrm{H}, \mathrm{H}_{23}\right.$ or $\left.\mathrm{H}_{25}\right) ; 0.94(\mathrm{t}, \mathrm{J}=7.5$ $\mathrm{Hz}, 3 \mathrm{H}, \mathrm{H}_{6}$ or $\left.\mathrm{H}_{12}\right)$; 0.97 (t, J = $7.5 \mathrm{~Hz}, 3 \mathrm{H}, \mathrm{H}_{6}$ or $\left.\mathrm{H}_{12}\right)$; $1.21-1.38\left(\mathrm{~m}, 8 \mathrm{H}_{2} \mathrm{H}_{20}, \mathrm{H}_{21}, \mathrm{H}_{22}, \mathrm{H}_{24}\right.$ ); 1.45-1.50 (m, 1H, $\left.\mathrm{H}_{19}\right) ; 1.55\left(\mathrm{dq}, \mathrm{J}=7.5,13.0 \mathrm{~Hz}, 1 \mathrm{H}, \mathrm{H}_{5}\right.$ or $\left.\mathrm{H}_{11}\right) ; 1.57\left(\mathrm{~d}, \mathrm{~J}=13 \mathrm{~Hz}, \mathrm{H}_{7}\right) ; 1.66$ $\left(\mathrm{dq}, \mathrm{J}=7.5,13.0 \mathrm{~Hz}, 1 \mathrm{H}, \mathrm{H}_{5}\right.$ or $\left.\mathrm{H}_{11}\right) ; 1.70\left(\mathrm{~d}, \mathrm{~J}=13.0 \mathrm{~Hz}, \mathrm{H}_{7}\right) ; 1.76\left(\mathrm{dq}, \mathrm{J}=7.5,13.0 \mathrm{~Hz}, 1 \mathrm{H}, \mathrm{H}_{5}\right.$ or $\left.\mathrm{H}_{11}\right) ; 1.78\left(\mathrm{~d}, \mathrm{~J}=1.5 \mathrm{~Hz}, 3 \mathrm{H}, \mathrm{H}_{1}\right) ; 1.92\left(\mathrm{dq}, \mathrm{J}=7.5,13.0 \mathrm{~Hz}, 1 \mathrm{H}, \mathrm{H}_{5}\right.$ or $\left.\mathrm{H}_{11}\right) ; 1.98$ (dd, J = 7.6, $\left.13.5 \mathrm{~Hz}, 1 \mathrm{H}, \mathrm{H}_{18}\right) ; 1.99$ (dd, J = 7.6, $\left.13.5 \mathrm{~Hz}, 1 \mathrm{H}, \mathrm{H}_{18}\right) ; 5.12$ (q, J = 1.5 Hz, 1H, H $)$; 5.22 (dd, 1.5, 11.0 Hz, 1H, $\left.\mathrm{H}_{10}\right) ; 5.32\left(\mathrm{dd}, \mathrm{J}=1.5,18.0 \mathrm{~Hz}, 1 \mathrm{H}, \mathrm{H}_{10}\right) ; 5.67$ (dd, J = 11.0, 18.0 Hz, 1H, H9); $6.10\left(\mathrm{~s}, 1 \mathrm{H}, \mathrm{H}_{13}\right) ; 7.11-7.26\left(\mathrm{~m}, 3 \mathrm{H}, \mathrm{H}_{16}, \mathrm{H}_{17}\right) ; 7.81\left(\mathrm{dd}, \mathrm{J}=1.0,8.5 \mathrm{~Hz}, \mathrm{H}_{15}\right) ;{ }^{13} \mathrm{C}$ NMR $(125$ $\left.\mathrm{MHz}, \mathrm{C}_{6} \mathrm{D}_{6}\right) \delta 7.6\left(\mathrm{C}_{6}\right) ; 8.6\left(\mathrm{C}_{12}\right) ; 11.2\left(\mathrm{C}_{25}\right) ; 14.8\left(\mathrm{C}_{23}\right) ; 23.9\left(\mathrm{C}_{1}\right) ; 26.4\left(\mathrm{C}_{22}\right) ; 30.0\left(\mathrm{C}_{24}\right) ; 30.6$ $\left(\mathrm{C}_{21}\right)$; $33.6\left(\mathrm{C}_{11}\right) ; 37.3\left(\mathrm{C}_{5}\right) ; 37.6\left(\mathrm{C}_{20}\right) ; 38.3\left(\mathrm{C}_{19}\right) ; 44.5\left(\mathrm{C}_{18}\right) ; 46.6\left(\mathrm{C}_{7}\right) ; 77.4\left(\mathrm{C}_{4}\right) ; 78.1\left(\mathrm{C}_{8}\right)$; $91.9\left(\mathrm{C}_{13}\right) ; 115.0\left(\mathrm{C}_{10}\right) ; 127.4\left(\mathrm{C}_{3}\right) ; 127.9\left(\mathrm{C}_{17}\right) ; 128.6\left(\mathrm{C}_{15}\right)$ 128.7 $\left(\mathrm{C}_{16}\right) ; 138.9\left(\mathrm{C}_{14}\right) ; 141.3\left(\mathrm{C}_{9}\right)$; $141.4\left(\mathrm{C}_{2}\right)$; EIMS m/z: $398\left(\mathrm{M}^{+}, 1\right), 397$ (4), 369 (5), 319 (9)292 (10), 276 (17), 263 (54), 247 (29), 182 (59), 181 (77), 164 (53), 163 (63), 151 (43), 149 (37), 135 (63), 124 (53), 122 (54), 105 (81), 83 (96), 69 (100); EIHRMS m/z: calcd for $\mathrm{C}_{27} \mathrm{H}_{41} \mathrm{O}_{2}\left(\mathrm{M}-\mathrm{H}^{+}\right), 397.31066$; found 397.30986; IR (NaCl, thin film) $\tilde{v}_{\max }\left(\mathrm{cm}^{-1}\right) ; 799,1017,1261,1458,2922$. 


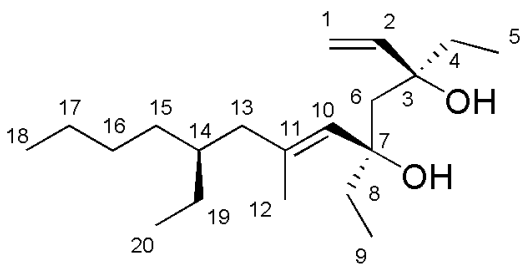

S-7

Preparation of $(3 R, 5 S, 9 R)-3,5,9-T r i e t h y l-7-m e t h y l t r i d e c a-1,6-d i e n e-3,5-d i o l ~ S-7: 25$ was converted to S-7 on a $0.1 \mathrm{mmol}$ scale using the procedure previously reported for the synthesis of 23, yielding S-7 (59\%) and S-13 (10\%) as colorless oils after column chromatography $\left(\mathrm{SiO}_{2}\right.$; hexanes/ether 9:1). $\mathrm{R}_{\mathrm{f}}(\mathbf{S}-7) 0.35$ (hexanes/ether 4:1); ${ }^{1} \mathrm{H}$ NMR (400 MHz, $\left.\mathrm{C}_{6} \mathrm{D}_{6}\right) \delta 0.88(\mathrm{t}, \mathrm{J}=$ $7.5 \mathrm{~Hz}, 3 \mathrm{H}, \mathrm{H}_{18}$ or $\mathrm{H}_{20}$ ); 0.90 (t, J = 7.5 Hz, $3 \mathrm{H}, \mathrm{H}_{18}$ or $\mathrm{H}_{20}$ ); 0.93 (t, J = 7.5 Hz, 3H, $\mathrm{H}_{5}$ or $\mathrm{H}_{9}$ ); 0.94 (t, J = 7.5 Hz, 3H, $\mathrm{H}_{5}$ or $\mathrm{H}_{9}$ ); 1.20-1.36 (m, 11H, H $\mathrm{H}_{4}, \mathrm{H}_{8}, \mathrm{H}_{14}, \mathrm{H}_{15}, \mathrm{H}_{16}, \mathrm{H}_{17}, \mathrm{H}_{19}$ ); 1.49 (dq, J $=7.5,13.0 \mathrm{~Hz}, 1 \mathrm{H}, \mathrm{H}_{4}$ or $\left.\mathrm{H}_{8}\right) ; 1.52\left(\mathrm{dq}, \mathrm{J}=7.5,13.0 \mathrm{~Hz}, 1 \mathrm{H}, \mathrm{H}_{4}\right.$ or $\left.\mathrm{H}_{8}\right) ; 1.71(\mathrm{~d}, \mathrm{~J}=14.0 \mathrm{~Hz}$, $\left.\mathrm{H}_{6}\right) ; 1.78\left(\mathrm{~d}, \mathrm{~J}=1 \mathrm{~Hz}, 3 \mathrm{H}, \mathrm{H}_{12}\right) ; 1.79\left(\mathrm{~d}, \mathrm{~J}=14.0 \mathrm{~Hz}, \mathrm{H}_{6}\right) ; 1.88\left(\mathrm{~d}, \mathrm{~J}=7.6 \mathrm{~Hz}, 2 \mathrm{H}, \mathrm{H}_{13}\right) ; 2.62(\mathrm{br}$ s, $\mathrm{OH}) ; 3.02(\mathrm{br} \mathrm{s}, \mathrm{OH}) ; 5.02\left(\mathrm{q}, \mathrm{J}=1.0 \mathrm{~Hz}, 1 \mathrm{H}, \mathrm{H}_{10}\right) ; 5.04\left(\mathrm{dd}, 2.0,10.8 \mathrm{~Hz}, 1 \mathrm{H}, \mathrm{H}_{1}\right) ; 5.34(\mathrm{dd}, \mathrm{J}$ $\left.=1.6,17.2 \mathrm{~Hz}, 1 \mathrm{H}, \mathrm{H}_{1}\right) ; 5.77\left(\mathrm{dd}, \mathrm{J}=10.8,17.2 \mathrm{~Hz}, 1 \mathrm{H}, \mathrm{H}_{2}\right) ;{ }^{13} \mathrm{C} \mathrm{NMR}\left(125 \mathrm{MHz}, \mathrm{C}_{6} \mathrm{D}_{6}\right) \delta 7.9$ $\left(\mathrm{C}_{5}\right) ; 8.5\left(\mathrm{C}_{9}\right) ; 11.1\left(\mathrm{C}_{20}\right) ; 14.8\left(\mathrm{C}_{18}\right) ; 23.9\left(\mathrm{C}_{12}\right) ; 26.1\left(\mathrm{C}_{17}\right) ; 29.8\left(\mathrm{C}_{19}\right) ; 30.6\left(\mathrm{C}_{16}\right) ; 33.5\left(\mathrm{C}_{15}\right)$; $36.8\left(\mathrm{C}_{4}\right) ; 37.3\left(\mathrm{C}_{8}\right) ; 37.8\left(\mathrm{C}_{14}\right) ; 46.8\left(\mathrm{C}_{13}\right) ; 51.4\left(\mathrm{C}_{6}\right) ; 76.7\left(\mathrm{C}_{7}\right) ; 77.9\left(\mathrm{C}_{3}\right) ; 111.7\left(\mathrm{C}_{1}\right) ; 133.1$

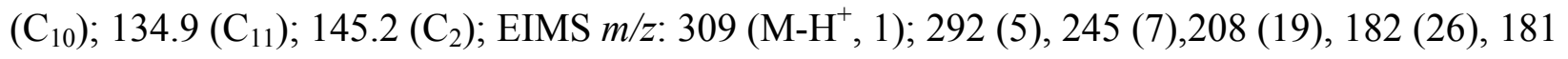
(83), 179 (78), 161 (28), 151 (63), 133 (42), 123 (47), 111 (72), 110 (74), 96 (78), 85 (100); EIHRMS $m / z$ : calcd for $\mathrm{C}_{20} \mathrm{H}_{37} \mathrm{O}_{2}\left(\mathrm{M}-\mathrm{H}^{+}\right), 309.27935$; found 309.27902; IR ( $\mathrm{NaCl}$, thin film) $\tilde{v}_{\max }\left(\mathrm{cm}^{-1}\right) ; 801,1016,1261,2962,3357$. 


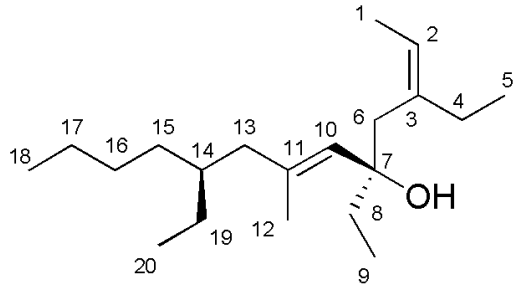

S-13

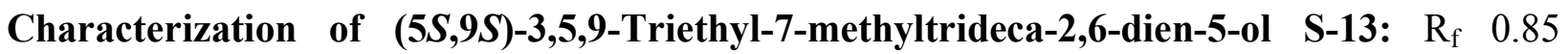
(hexanes/ether 4:1); ${ }^{1} \mathrm{H}$ NMR $\left(500 \mathrm{MHz}, \mathrm{C}_{6} \mathrm{D}_{6}\right) \delta 0.90\left(\mathrm{t}, \mathrm{J}=7.5 \mathrm{~Hz}, 3 \mathrm{H}, \mathrm{H}_{18}\right.$ or $\left.\mathrm{H}_{20}\right) ; 0.94(\mathrm{t}, \mathrm{J}=$ $7.5 \mathrm{~Hz}, 3 \mathrm{H}, \mathrm{H}_{18}$ or $\left.\mathrm{H}_{20}\right)$; 0.99 (t, J = $7.5 \mathrm{~Hz}, 3 \mathrm{H}, \mathrm{H}_{5}$ or $\left.\mathrm{H}_{9}\right) ; 1.03\left(\mathrm{t}, \mathrm{J}=7.5 \mathrm{~Hz}, 3 \mathrm{H}, \mathrm{H}_{5}\right.$ or $\mathrm{H}_{9}$ ); 1.26-1.38 (m, 9H, H $\left.\mathrm{H}_{14}, \mathrm{H}_{15}, \mathrm{H}_{16}, \mathrm{H}_{17}, \mathrm{H}_{19}\right) ; 1.51$ (d, J = 6.5 Hz, 3H, H $\mathrm{H}_{1}$; 1.58 (dq, J = 7.5, 13.0 $\left.\mathrm{Hz}, 1 \mathrm{H}, \mathrm{H}_{8}\right) ; 1.60\left(\mathrm{dq}, \mathrm{J}=7.5,13.0 \mathrm{~Hz}, 1 \mathrm{H}, \mathrm{H}_{8}\right) ; 1.93\left(\mathrm{~d}, \mathrm{~J}=1.0 \mathrm{~Hz}, 3 \mathrm{H}, \mathrm{H}_{12}\right) ; 1.95$ (d, J = 7.0 $\left.\mathrm{Hz}, 2 \mathrm{H}, \mathrm{H}_{13}\right) ; 2.18\left(\mathrm{q}, \mathrm{J}=7.5 \mathrm{~Hz}, 2 \mathrm{H}, \mathrm{H}_{4}\right) ; 2.24$ (d, J = 8.5 Hz, 1H, H6); 2.37 (d, J = 8.5 Hz, 1H,

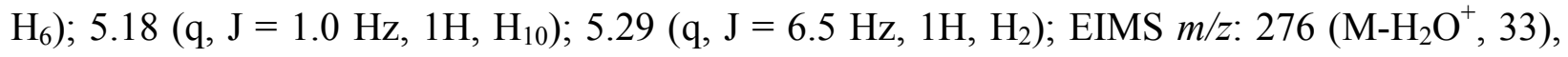
247 (45), 205 (28), 177 (55), 163 (100), 149 (88), 135 (96), 121 (91), 107 (93), 93 (87), 83 (88), 69 (89).

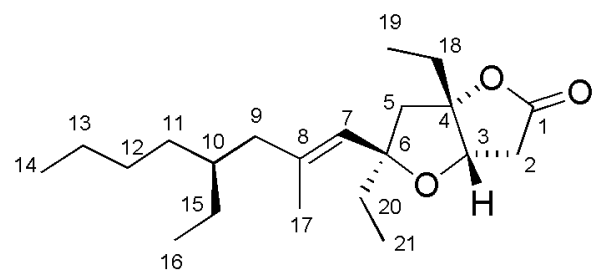

26

Preparation of 11,12-Dihydroplakortone B 26: S-7 was converted to 26 on a $0.01 \mathrm{mmol}$ scale using the procedure reported for the synthesis of $\mathbf{1}$, yielding $26(75 \%)$ as a colorless oil after column chromatography $\left(\mathrm{SiO}_{2}\right.$; hexanes/ether 4:1), $\mathrm{R}_{\mathrm{f}} 0.2$ (hexanes/ether 4:1); ${ }^{1} \mathrm{H}$ NMR (500 $\left.\mathrm{MHz} \mathrm{CDCl}_{3}\right) \delta 0.84\left(\mathrm{t}, \mathrm{J}=7.5 \mathrm{~Hz}, 3 \mathrm{H}, \mathrm{H}_{16}\right) ; 0.89\left(\mathrm{t}, \mathrm{J}=7.5 \mathrm{~Hz}, 3 \mathrm{H}, \mathrm{H}_{14}\right) ; 0.90(\mathrm{t}, \mathrm{J}=7.5 \mathrm{~Hz}$, $\mathrm{H}_{21}$ ); 0.98 (t, J = 7.5 Hz, 3H, H $\left.\mathrm{H}_{19}\right) ; 1.17-1.32$ (m, 9H, $\left.\mathrm{H}_{10}, \mathrm{H}_{11}, \mathrm{H}_{12}, \mathrm{H}_{13}, \mathrm{H}_{15}\right) ; 1.69$ (dq, J = 7.5, $13.0 \mathrm{~Hz}, 1 \mathrm{H}, \mathrm{H}_{18}$ or $\left.\mathrm{H}_{20}\right) ; 1.71$ (d, $\left.\mathrm{J}=1 \mathrm{~Hz}, 3 \mathrm{H}, \mathrm{H}_{17}\right) ; 1.72\left(\mathrm{dq}, \mathrm{J}=7.5,13.0 \mathrm{~Hz}, 1 \mathrm{H}, \mathrm{H}_{18}\right.$ or $\left.\mathrm{H}_{20}\right)$; 1.79 (dq, $\mathrm{J}=7.5,13.0 \mathrm{~Hz}, 1 \mathrm{H}, \mathrm{H}_{18}$ or $\left.\mathrm{H}_{20}\right) ; 1.84$ (dq, J = 7.5, $13.0 \mathrm{~Hz}, 1 \mathrm{H}, \mathrm{H}_{18}$ or $\mathrm{H}_{20}$ ); 1.86 (dd, $\left.\mathrm{J}=7,13.5 \mathrm{~Hz}, 1 \mathrm{H}, \mathrm{H}_{9}\right) ; 1.93\left(\mathrm{dd}, \mathrm{J}=7.0,13.5 \mathrm{~Hz}, 1 \mathrm{H}, \mathrm{H}_{9}\right) ; 2.17\left(\mathrm{~d}, \mathrm{~J}=14.0 \mathrm{~Hz}, 1 \mathrm{H}, \mathrm{H}_{5}\right) ; 2.27$ 
$\left(\mathrm{d}, \mathrm{J}=14.0 \mathrm{~Hz}, 1 \mathrm{H}, \mathrm{H}_{5}\right) ; 2.68\left(\mathrm{dd}, \mathrm{J}=1.0,18.5 \mathrm{~Hz}, 1 \mathrm{H}, \mathrm{H}_{2}\right) ; 2.70\left(\mathrm{dd}, \mathrm{J}=5.0,18.5 \mathrm{~Hz}, 1 \mathrm{H}, \mathrm{H}_{2}\right) ;$ $4.23\left(\mathrm{dd}, \mathrm{J}=1.0,5.0 \mathrm{~Hz}, 1 \mathrm{H}, \mathrm{H}_{3}\right) ; 5.08\left(\mathrm{q}, \mathrm{J}=1.0 \mathrm{~Hz}, 1 \mathrm{H}, \mathrm{H}_{7}\right) ;{ }^{13} \mathrm{C} \mathrm{NMR}\left(125 \mathrm{MHz}, \mathrm{CDCl}_{3}\right) \delta$ $8.6\left(\mathrm{C}_{19}\right)$; $9.1\left(\mathrm{C}_{21}\right) ; 10.8\left(\mathrm{C}_{16}\right) ; 14.4\left(\mathrm{C}_{14}\right) ; 16.7\left(\mathrm{C}_{17}\right) ; 23.4\left(\mathrm{C}_{13}\right) ; 25.8\left(\mathrm{C}_{18}\right) ; 29.0\left(\mathrm{C}_{15}\right) ; 30.6$ $\left(\mathrm{C}_{12}\right) ; 32.7\left(\mathrm{C}_{20}\right) ; 34.0\left(\mathrm{C}_{11}\right) ; 36.8\left(\mathrm{C}_{10}\right) ; 37.0\left(\mathrm{C}_{2}\right) ; 45.8\left(\mathrm{C}_{9}\right) ; 49.2\left(\mathrm{C}_{5}\right) ; 79.8\left(\mathrm{C}_{3}\right) ; 87.2\left(\mathrm{C}_{6}\right) ; 97.5$ $\left(\mathrm{C}_{4}\right) ; 129.9\left(\mathrm{C}_{7}\right) ; 137.9\left(\mathrm{C}_{8}\right) ; \operatorname{ESMS} m / z: 359\left(\mathrm{M}+\mathrm{Na}^{+}\right), 337\left(\mathrm{M}+\mathrm{H}^{+}\right)$

\section{Synthesis of 9,10-Epiplakortone Core}

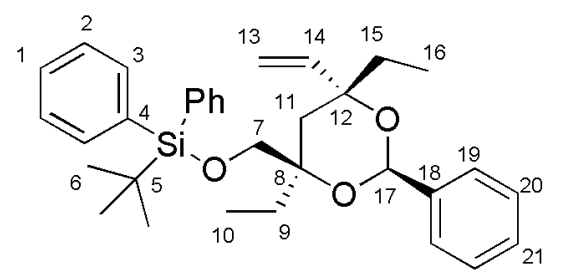

S-8

Preparation of (4S,6S)-tert-Butyl-(4,6-diethyl-2-phenyl-6-vinyl-1,3-dioxan-4ylmethoxy)diphenylsilane S-8: Diol 8 was converted to S-8 on a 1.7 mmol scale using the procedure previously reported for the synthesis of S-3, yielding $\mathbf{S - 8}(98 \%)$ as a colorless oil after column chromatography $\left(\mathrm{SiO}_{2}\right.$; hexanes/ether 50:1), $\mathrm{R}_{\mathrm{f}} 0.85$ (hexanes/ether 19:1); ${ }^{1} \mathrm{H}$ NMR (300 $\left.\mathrm{MHz} \mathrm{CDCl}_{3}\right) \delta 0.84\left(\mathrm{t}, \mathrm{J}=7.5 \mathrm{~Hz}, 3 \mathrm{H}, \mathrm{H}_{16}\right.$ or $\left.\mathrm{H}_{10}\right) ; 0.91\left(\mathrm{t}, \mathrm{J}=7.5 \mathrm{~Hz}, 3 \mathrm{H}, \mathrm{H}_{16}\right.$ or $\left.\mathrm{H}_{10}\right) ; 1.10$ (s, $\left.9 \mathrm{H}, \mathrm{H}_{6}\right) ; 1.59\left(\mathrm{~m}, 2 \mathrm{H}, \mathrm{H}_{9}\right.$ or $\left.\mathrm{H}_{15}\right) ; 1.83\left(\mathrm{~m}, 3 \mathrm{H}, \mathrm{H}_{11}\right.$ and either $\mathrm{H}_{9}$ or $\left.\mathrm{H}_{15}\right) ; 2.24(\mathrm{dq}, \mathrm{J}=7.5,14.7$ $\mathrm{Hz}, \mathrm{H}_{9}$ or $\left.\mathrm{H}_{15}\right) ; 3.46\left(\mathrm{~d}, \mathrm{~J}=10.0 \mathrm{~Hz}, \mathrm{H}_{7}\right) ; 3.69\left(\mathrm{~d}, \mathrm{~J}=10.0 \mathrm{~Hz}, \mathrm{H}_{7}\right) ; 5.28(\mathrm{dd}, \mathrm{J}=1.2,17.4 \mathrm{~Hz}$, $\left.1 \mathrm{H}, \mathrm{H}_{13}\right) ; 5.33\left(\mathrm{dd}, \mathrm{J}=1.2,11.1 \mathrm{~Hz}, 1 \mathrm{H}, \mathrm{H}_{13}\right) ; 5.82\left(\mathrm{~s}, 1 \mathrm{H}, \mathrm{H}_{17}\right) ; 5.91(\mathrm{dd}, \mathrm{J}=11.1,17.4 \mathrm{~Hz}, 1 \mathrm{H}$, $\left.\mathrm{H}_{14}\right)$; 7.3-7.46 (m, 9H, $\left.\mathrm{H}_{1}, \mathrm{H}_{2}, \mathrm{H}_{20}, \mathrm{H}_{21}\right)$; 7.5-7.56 (m, 2H, $\left.\mathrm{H}_{19}\right)$; 7.66-7.72 (m, 4H, $\left.\mathrm{H}_{3}\right)$; ${ }^{13} \mathrm{C} \mathrm{NMR}$ $\left(75 \mathrm{MHz}, \mathrm{CDCl}_{3}\right) \delta 7.2\left(\mathrm{C}_{16}\right) ; 7.6\left(\mathrm{C}_{10}\right) ; 19.6\left(\mathrm{C}_{5}\right) ; 23.6\left(\mathrm{C}_{9}\right) ; 27.1\left(\mathrm{C}_{6}\right) ; 38.0\left(\mathrm{C}_{15}\right) ; 39.0\left(\mathrm{C}_{11}\right)$; $69.3\left(\mathrm{C}_{12}\right) ; 76.7\left(\mathrm{C}_{8}\right) ; 77.6\left(\mathrm{C}_{7}\right) ; 89.7\left(\mathrm{C}_{17}\right) ; 114.9\left(\mathrm{C}_{13}\right) ; 126.4\left(\mathrm{C}_{21}\right) ; 127.8\left(\mathrm{C}_{20}\right) ; 128.3\left(\mathrm{C}_{3}\right)$; $128.5\left(\mathrm{C}_{1}\right) ; 129.8\left(\mathrm{C}_{2}\right) ; 133.7\left(\mathrm{C}_{4}\right) ; 135.9\left(\mathrm{C}_{19}\right) ; 139.9\left(\mathrm{C}_{18}\right) ; 141.9\left(\mathrm{C}_{14}\right)$; EIMS m/z: $514\left(\mathrm{M}^{+}, 1\right)$, 513 (2), 433 (4), 379 (15), 351 (32), 333 (19), 269 (62), 239 (28), 199 (58), 191 (62), 163 (32), 139 (100), 135 (92), 123 (48), 105 (60), 91 (30), 77 (62); EIHRMS m/z: calcd for 513.2825; found 513.2811; IR $\left(\mathrm{NaCl}\right.$, thin film) $\tilde{v}_{\max }\left(\mathrm{cm}^{-1}\right) ; 701,740,823,924,1021,1113,1360,1428$, $1461,1472,2857,2880,2991,2964,3070$. 


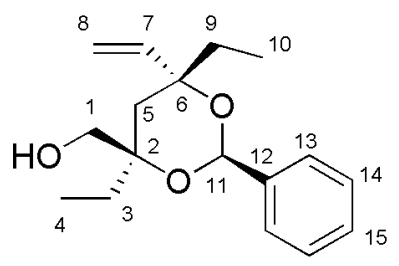

S-9

Preparation of $(4 S, 6 S)$-(4,6-Diethyl-2-phenyl-6-vinyl-1,3-dioxan-4-yl)methanol S-9: Ether S8 was converted to $\mathbf{S - 9}$ on a $1.7 \mathrm{mmol}$ scale using the procedure previously reported for the synthesis of S-5, yielding S-9 (94\%) as a colorless oil after column chromatography $\left(\mathrm{SiO}_{2}\right.$; hexanes/ether 4:1), $\mathrm{R}_{\mathrm{f}} 0.2$ (hexanes/ether 4:1); ${ }^{1} \mathrm{H} \mathrm{NMR}\left(400 \mathrm{MHz}, \mathrm{CDCl}_{3}\right) \delta 0.90(\mathrm{t}, \mathrm{J}=7.5 \mathrm{~Hz}$, $3 \mathrm{H}, \mathrm{H}_{4}$ or $\left.\mathrm{H}_{10}\right) ; 0.92\left(\mathrm{t}, \mathrm{J}=7.5 \mathrm{~Hz}, 3 \mathrm{H}, \mathrm{H}_{4}\right.$ or $\left.\mathrm{H}_{10}\right) ; 1.60\left(\mathrm{~d}, \mathrm{~J}=14.0 \mathrm{~Hz}, 1 \mathrm{H}, \mathrm{H}_{5}\right) ; 1.64(\mathrm{dq}, \mathrm{J}=$ 7.5, $14.4 \mathrm{~Hz}, 1 \mathrm{H}, \mathrm{H}_{9}$ or $\left.\mathrm{H}_{3}\right) ; 1.66\left(\mathrm{dq}, \mathrm{J}=7.5,14.4 \mathrm{~Hz}, 1 \mathrm{H}, \mathrm{H}_{9}\right.$ or $\left.\mathrm{H}_{3}\right) ; 1.77$ (dq, J = 7.5, $14.4 \mathrm{~Hz}$, $1 \mathrm{H}, \mathrm{H}_{9}$ or $\left.\mathrm{H}_{3}\right) ; 2.03$ (dq, J = 7.5, $14.4 \mathrm{~Hz}, 1 \mathrm{H}, \mathrm{H}_{9}$ or $\left.\mathrm{H}_{3}\right) ; 2.05(\mathrm{~m}, 1 \mathrm{H}, \mathrm{OH}) ; 2.09$ (d, J = $14.0 \mathrm{~Hz}$, $\left.1 \mathrm{H}, \mathrm{H}_{5}\right) ; 3.32\left(\mathrm{dd}, \mathrm{J}=9.2,11.2 \mathrm{~Hz}, 1 \mathrm{H}, \mathrm{H}_{1}\right) ; 3.64\left(\mathrm{dd}, \mathrm{J}=3.6,11.2 \mathrm{~Hz}, 1 \mathrm{H}, \mathrm{H}_{1}\right) ; 5.29(\mathrm{dd}, \mathrm{J}=$ 1.2, $\left.17.6 \mathrm{~Hz}, 1 \mathrm{H}, \mathrm{H}_{8}\right) ; 5.33\left(\mathrm{dd}, \mathrm{J}=1.2,10.8 \mathrm{~Hz}, 1 \mathrm{H}, \mathrm{H}_{8}\right) ; 5.88\left(\mathrm{~s}, 1 \mathrm{H}, \mathrm{H}_{11}\right) ; 5.89$ (dd, J = 10.8, $\left.17.6 \mathrm{~Hz}, 1 \mathrm{H}, \mathrm{H}_{7}\right) ; 7.33-7.42\left(\mathrm{~m}, 3 \mathrm{H}, \mathrm{H}_{14}, \mathrm{H}_{15}\right) ; 7.52-7.56\left(\mathrm{~m}, 2 \mathrm{H}, \mathrm{H}_{13}\right) ;{ }^{13} \mathrm{C} \mathrm{NMR}(75 \mathrm{MHz}$, $\left.\mathrm{CDCl}_{3}\right) \delta 7.1\left(\mathrm{C}_{4}\right) ; 8.0\left(\mathrm{C}_{10}\right) ; 25.1\left(\mathrm{C}_{3}\right) ; 34.8\left(\mathrm{C}_{9}\right) ; 38.0\left(\mathrm{C}_{5}\right) ; 68.0\left(\mathrm{C}_{2}\right) ; 76.6\left(\mathrm{C}_{1}\right) ; 76.9\left(\mathrm{C}_{6}\right) ; 89.9$ $\left(\mathrm{C}_{11}\right) ; 115.3\left(\mathrm{C}_{8}\right) ; 126.4\left(\mathrm{C}_{13}\right) ; 128.5\left(\mathrm{C}_{14}\right) ; 128.9\left(\mathrm{C}_{15}\right) ; 139.6\left(\mathrm{C}_{12}\right) ; 141.7\left(\mathrm{C}_{7}\right)$; EIMS m/z: 276 $\left(\mathrm{M}^{+}, 1\right), 275$ (3), 177 (8), 141 (49), 139 (100), 123 (42), 105 (86), 77 (68); EIHRMS m/z: calcd for $\mathrm{C}_{19} \mathrm{H}_{23} \mathrm{O}_{2}, 276.17255$; found 276.16998; IR (NaCl, thin film) $\tilde{v}_{\max }\left(\mathrm{cm}^{-1}\right) ; 924,1089,1173$, $1377,1457,2886,3444$.

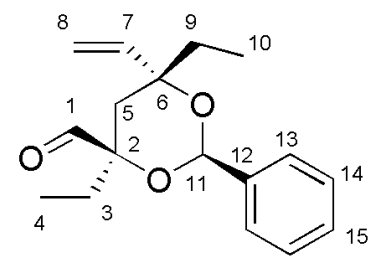

27

Preparation of (4S,6S)-4,6-Diethyl-2-phenyl-6-vinyl-1,3-dioxane-4-carbaldehyde 27: Alcohol S-9 was converted to 27 on a $2.0 \mathrm{mmol}$ scale using the procedure previously reported 
for the synthesis of 10, yielding $27(83 \%)$ as a colorless oil after column chromatography $\left(\mathrm{SiO}_{2}\right.$; hexanes/ether 19:1), $\mathrm{R}_{\mathrm{f}} 0.7$ (hexanes/ether 9:1); ${ }^{1} \mathrm{H}$ NMR $\left(300 \mathrm{MHz}, \mathrm{CDCl}_{3}\right) \delta 0.90$ (t, J = 7.5 $\mathrm{Hz}, 3 \mathrm{H}, \mathrm{H}_{4}$ or $\left.\mathrm{H}_{10}\right) ; 0.92\left(\mathrm{t}, \mathrm{J}=7.5 \mathrm{~Hz}, 3 \mathrm{H}, \mathrm{H}_{4}\right.$ or $\left.\mathrm{H}_{10}\right) ; 1.59$ (dq, J = 7.5, $14.4 \mathrm{~Hz}, 1 \mathrm{H}, \mathrm{H}_{9}$ or $\left.\mathrm{H}_{3}\right)$; $1.64\left(\mathrm{dq}, \mathrm{J}=7.5,14.4 \mathrm{~Hz}, 1 \mathrm{H}, \mathrm{H}_{9}\right.$ or $\left.\mathrm{H}_{3}\right) ; 1.79$ (d, J = 14.1 Hz, 1H, H5); 1.85 (dq, J = 7.5, 14.4 $\mathrm{Hz}, 1 \mathrm{H}, \mathrm{H}_{9}$ or $\left.\mathrm{H}_{3}\right) ; 1.95\left(\mathrm{~d}, \mathrm{~J}=14.1 \mathrm{~Hz}, 1 \mathrm{H}, \mathrm{H}_{5}\right) ; 2.20$ (ddq, $\left.\mathrm{J}=1.2,7.5,14.4 \mathrm{~Hz}, 1 \mathrm{H}, \mathrm{H}_{3}\right) ; 5.30$ $\left(\mathrm{dd}, \mathrm{J}=1.2,17.4 \mathrm{~Hz}, 1 \mathrm{H}, \mathrm{H}_{8}\right) ; 5.35\left(\mathrm{dd}, \mathrm{J}=1.2,11.1 \mathrm{~Hz}, 1 \mathrm{H}, \mathrm{H}_{8}\right) ; 5.85(\mathrm{dd}, \mathrm{J}=11.1,17.4 \mathrm{~Hz}$, $\left.1 \mathrm{H}, \mathrm{H}_{7}\right) ; 5.90\left(\mathrm{~s}, 1 \mathrm{H}, \mathrm{H}_{11}\right) ; 7.38-7.46\left(\mathrm{~m}, 3 \mathrm{H}, \mathrm{H}_{14}, \mathrm{H}_{15}\right) ; 7.58-7.61\left(\mathrm{~m}, 2 \mathrm{H}, \mathrm{H}_{13}\right) ; 9.69$ (d, J = 1.2 $\left.\mathrm{Hz}, 1 \mathrm{H}, \mathrm{H}_{1}\right) ;{ }^{13} \mathrm{C} \mathrm{NMR}\left(75 \mathrm{MHz}, \mathrm{CDCl}_{3}\right) \delta 7.2\left(\mathrm{C}_{4}\right) ; 7.9\left(\mathrm{C}_{10}\right) ; 26.4\left(\mathrm{C}_{3}\right) ; 35.5\left(\mathrm{C}_{9}\right) ; 36.8\left(\mathrm{C}_{5}\right)$; $77.6\left(\mathrm{C}_{6}\right) ; 81.5\left(\mathrm{C}_{2}\right) ; 91.1\left(\mathrm{C}_{8}\right) ; 126.4\left(\mathrm{C}_{13}\right) ; 128.6\left(\mathrm{C}_{14}\right) ; 129.1\left(\mathrm{C}_{15}\right) ; 139.0\left(\mathrm{C}_{12}\right) ; 140.1\left(\mathrm{C}_{7}\right)$; $205.0\left(\mathrm{C}_{1}\right)$; ESMS m/z: $297\left(\mathrm{M}+\mathrm{Na}^{+}\right), 275\left(\mathrm{M}+\mathrm{H}^{+}\right)$.

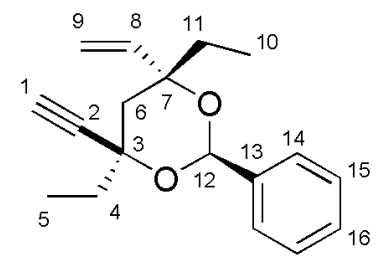

S-10

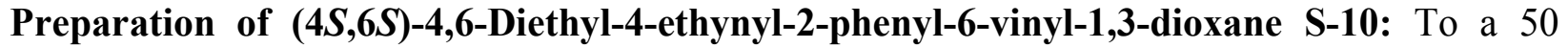
$\mathrm{mL}$, three-necked, round-bottomed flask equipped with a magnetic stirring bar and a lowtemperature thermometer was added dimethyl (diazomethyl)phosphonate (290 mg, $1.95 \mathrm{mmol}$ ). The flask was placed under an argon atmosphere, and THF $(20 \mathrm{~mL})$ was added via syringe. The mixture was cooled to $-55^{\circ} \mathrm{C}$ with a constant temperature bath and potassium tert-butoxide (1.95 mmol, $1 \mathrm{M}$ solution in THF, $1.95 \mathrm{~mL}$ ) was added dropwise via syringe. The solution was stirred for $30 \mathrm{~min}$ and 27 (438 mg, $1.622 \mathrm{mmol})$ in THF (2 mL) was added dropwise via syringe. The solution was stirred at $-55^{\circ} \mathrm{C}$ for $16 \mathrm{~h}$ until a precipitate formed, then warmed to $23{ }^{\circ} \mathrm{C}$ and stirred for a further $2 \mathrm{~h}$. Water $(10 \mathrm{~mL})$ and ether $(50 \mathrm{~mL})$ were added, the mixture was transferred to a separatory flask, and the layers separated. The aqueous layer was extracted with ether $(3 \times 10 \mathrm{~mL})$ and the combined organic extracts washed with saturated brine $(20 \mathrm{~mL})$ and water $(20 \mathrm{~mL})$, dried over $\mathrm{MgSO}_{4}$, filtered and the solvent removed by rotary evaporation. The crude oil was purified by column chromatography $\left(\mathrm{SiO}_{2}\right.$ : hexanes/ether 50:1) to yield $\mathbf{S - 1 0}$ (410 
mg, 95\%) as a white solid, mp $35^{\circ} \mathrm{C}, \mathrm{R}_{\mathrm{f}} 0.75$ (hexanes/ether 19:1); ${ }^{1} \mathrm{H}$ NMR $\left(300 \mathrm{MHz}, \mathrm{CDCl}_{3}\right)$ $\delta 0.91\left(\mathrm{t}, \mathrm{J}=7.5 \mathrm{~Hz}, 3 \mathrm{H}, \mathrm{H}_{5}\right.$ or $\left.\mathrm{H}_{10}\right) ; 1.09\left(\mathrm{t}, \mathrm{J}=7.5 \mathrm{~Hz}, 3 \mathrm{H}, \mathrm{H}_{5}\right.$ or $\left.\mathrm{H}_{10}\right) ; 1.64(\mathrm{dq}, \mathrm{J}=7.5,14.4$ $\mathrm{Hz}, 1 \mathrm{H}, \mathrm{H}_{4}$ or $\left.\mathrm{H}_{11}\right) ; 1.74\left(\mathrm{dq}, \mathrm{J}=7.5,14.4 \mathrm{~Hz}, 1 \mathrm{H}, \mathrm{H}_{4}\right.$ or $\left.\mathrm{H}_{11}\right) ; 1.77$ (dq, J = 7.5, $14.4 \mathrm{~Hz}, 1 \mathrm{H}, \mathrm{H}_{4}$ or $\left.\mathrm{H}_{11}\right) ; 2.01$ (d, J = 14.1 Hz, 1H, $\left.\mathrm{H}_{6}\right) ; 2.29$ (d, J = 14.1 Hz, 1H, $\left.\mathrm{H}_{6}\right) ; 2.31$ (dq, J = 7.5, $14.4 \mathrm{~Hz}$, $\left.1 \mathrm{H}, \mathrm{H}_{4}\right) ; 2.50\left(\mathrm{~s}, 1 \mathrm{H}, \mathrm{H}_{1}\right) ; 5.27\left(\mathrm{dd}, \mathrm{J}=1.5,15.5 \mathrm{~Hz}, 1 \mathrm{H}, \mathrm{H}_{9}\right) ; 5.31\left(\mathrm{dd}, \mathrm{J}=1.5,10.5 \mathrm{~Hz}, 1 \mathrm{H}, \mathrm{H}_{9}\right)$; 5.77 (s, 1H, $\left.\mathrm{H}_{12}\right) ; 5.85$ (dd, J = 10.5, $\left.15.5 \mathrm{~Hz}, 1 \mathrm{H}, \mathrm{H}_{8}\right) ; 7.38-7.46\left(\mathrm{~m}, 3 \mathrm{H}, \mathrm{H}_{15}, \mathrm{H}_{16}\right)$; 7.58-7.61 $\left(\mathrm{m}, 2 \mathrm{H}, \mathrm{H}_{14}\right) ;{ }^{13} \mathrm{C} \mathrm{NMR}\left(75 \mathrm{MHz}, \mathrm{CDCl}_{3}\right) \delta 7.3\left(\mathrm{C}_{5}\right) ; 8.9\left(\mathrm{C}_{10}\right) ; 30.0\left(\mathrm{C}_{11}\right) ; 37.0\left(\mathrm{C}_{4}\right) ; 41.4\left(\mathrm{C}_{6}\right)$; $72.2\left(\mathrm{C}_{1}\right) ; 72.8\left(\mathrm{C}_{3}\right) ; 77.3\left(\mathrm{C}_{7}\right) ; 86.7\left(\mathrm{C}_{2}\right) ; 90.6\left(\mathrm{C}_{12}\right) ; 115.3\left(\mathrm{C}_{9}\right) ; 126.6\left(\mathrm{C}_{14}\right) ; 128.4\left(\mathrm{C}_{16}\right) ; 128.9$ $\left(\mathrm{C}_{15}\right)$; $139.1\left(\mathrm{C}_{13}\right) ; 141.3\left(\mathrm{C}_{8}\right)$; ESMS m/z: $293\left(\mathrm{M}+\mathrm{Na}^{+}\right), 271\left(\mathrm{M}+\mathrm{H}^{+}\right)$; IR $(\mathrm{NaCl}$, thin film $) \tilde{\mathrm{v}}_{\max }$ $\left(\mathrm{cm}^{-1}\right) ; 920,1377,1456,2972,3028,3293$.

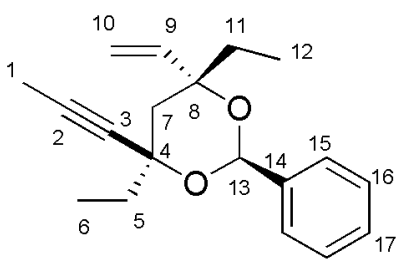

28

Preparation of (4S,6S)-4,6-Diethyl-2-phenyl-4-prop-1-ynyl-6-vinyl-1,3-dioxane 28: S-10 was converted to $\mathbf{2 8}$ on a $1.6 \mathrm{mmol}$ scale using the procedure previously reported for the synthesis of 11, yielding 28 (95\%) as a colorless oil after column chromatography $\left(\mathrm{SiO}_{2}\right.$; hexanes/ether 50:1), $\mathrm{R}_{\mathrm{f}} 0.75$ (hexanes/ether 19:1). $\mathrm{R}_{\mathrm{f}} 0.75$ (hexanes/ether 19:1); ${ }^{1} \mathrm{H}$ NMR $\left(300 \mathrm{MHz}, \mathrm{CDCl}_{3}\right) \delta 0.90(\mathrm{t}$, $\mathrm{J}=7.5 \mathrm{~Hz}, 3 \mathrm{H}, \mathrm{H}_{6}$ or $\left.\mathrm{H}_{12}\right) ; 1.06\left(\mathrm{t}, \mathrm{J}=7.5 \mathrm{~Hz}, 3 \mathrm{H}, \mathrm{H}_{6}\right.$ or $\left.\mathrm{H}_{12}\right) ; 1.6-1.75\left(\mathrm{~m}, 3 \mathrm{H}, \mathrm{H}_{11}, \mathrm{H}_{5}\right) ; 1.84(\mathrm{~s}$, $\left.3 \mathrm{H}, \mathrm{H}_{1}\right) ; 1.95\left(\mathrm{~d}, \mathrm{~J}=14.0 \mathrm{~Hz}, 1 \mathrm{H}, \mathrm{H}_{7}\right) ; 2.24\left(\mathrm{~d}, \mathrm{~J}=14.0 \mathrm{~Hz}, 1 \mathrm{H}, \mathrm{H}_{7}\right) ; 2.30$ (dq, J = 7.5, $14.1 \mathrm{~Hz}$, $\left.1 \mathrm{H}, \mathrm{H}_{5}\right) ; 5.24\left(\mathrm{dd}, \mathrm{J}=1.2,17.4 \mathrm{~Hz}, 1 \mathrm{H}, \mathrm{H}_{10}\right) ; 5.28\left(\mathrm{dd}, \mathrm{J}=1.2,11.1 \mathrm{~Hz}, 1 \mathrm{H}, \mathrm{H}_{10}\right) ; 5.74(\mathrm{~s}, 1 \mathrm{H}$, $\left.\mathrm{H}_{13}\right) ; 5.84\left(\mathrm{dd}, \mathrm{J}=11.1,17.4 \mathrm{~Hz}, 1 \mathrm{H}, \mathrm{H}_{10}\right) ; 7.29-7.38\left(\mathrm{~m}, 3 \mathrm{H}, \mathrm{H}_{16}, \mathrm{H}_{17}\right) ; 7.2-7.58\left(\mathrm{~m}, 2 \mathrm{H}, \mathrm{H}_{15}\right)$; ${ }^{13} \mathrm{C}$ NMR $\left(75 \mathrm{MHz}, \mathrm{CDCl}_{3}\right) \delta 3.9\left(\mathrm{C}_{1}\right) ; 7.3\left(\mathrm{C}_{6}\right) ; 9.1\left(\mathrm{C}_{12}\right) ; 29.8\left(\mathrm{C}_{5}\right) ; 37.3\left(\mathrm{C}_{11}\right) ; 42.0\left(\mathrm{C}_{7}\right) ; 73.3$ $\left(\mathrm{C}_{4}\right) ; 76.4\left(\mathrm{C}_{6}\right) ; 80.1\left(\mathrm{C}_{2}\right) ; 82.4\left(\mathrm{C}_{3}\right) ; 90.4\left(\mathrm{C}_{13}\right) ; 115.1\left(\mathrm{C}_{10}\right) ; 126.6\left(\mathrm{C}_{17}\right) ; 128.4\left(\mathrm{C}_{16}\right) ; 128.7$

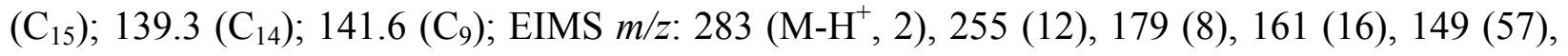
135 (52), 119 (51), 105 (82), 93 (48), 79 (55), 67 (100). 


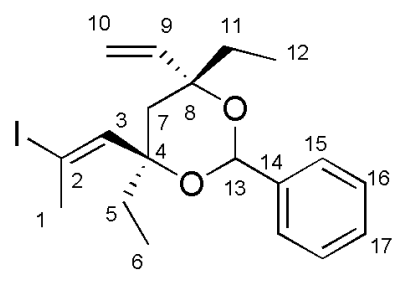

29

Preparation of (4S,6S)-4,6-Diethyl-4-(2-iodopropenyl)-2-phenyl-6-vinyl-1,3-dioxane 29: To a 25-mL, argon-filled, two-necked, round-bottomed flask equipped with a magnetic stirring bar was added palladium(II) acetate $(0.02 \mathrm{mmol}, 6 \mathrm{mg})$ and tricyclohexylphosphine (0.06 mmol, 14 $\mathrm{mg})$. The flask was evacuated and filled with argon three times. Freshly distilled hexane $(3 \mathrm{~mL})$ was added and the solution stirred for $10 \mathrm{~min}$ until the solids had dissolved. 28 (70 $\mathrm{mg}, 0.25$ $\mathrm{mmol}$ ) in hexane was added via syringe and the mixture cooled to $-35^{\circ} \mathrm{C}$. Tributyltin hydride $(0.49 \mathrm{mmol}, 144 \mathrm{mg}, 130 \mu \mathrm{L})$ was added via syringe. The reaction was stirred for $15 \mathrm{~h}$, then immediately transferred to a silica gel column and rapidly eluted with hexane until the excess $\mathrm{Bu}_{3} \mathrm{SnH} /\left(\mathrm{Bu}_{3} \mathrm{Sn}\right)_{2}$ is removed, followed by elution with hexane/ether (10:1) to obtain a colorless oil which was a 1:1:1 mixture of starting acetylene 28, product S-11 and distannane. S-11 was not purified, and the crude stannane converted to $\mathbf{2 9}$ using the procedure previously reported for the synthesis of $\mathbf{1 4}$ yielding $\mathbf{2 9}$ (29\% over 2 steps) as a colorless oil after column chromatography $\left(\mathrm{SiO}_{2}\right.$; hexanes/ether 500:1), $\mathrm{R}_{\mathrm{f}} 0.8$ (hexanes/ether 19:1); ${ }^{1} \mathrm{H}$ NMR (400 MHz, $\left.\mathrm{CDCl}_{3}\right) \delta 0.87\left(\mathrm{t}, \mathrm{J}=7.5 \mathrm{~Hz}, 3 \mathrm{H}, \mathrm{H}_{12}\right) ; 0.89\left(\mathrm{t}, \mathrm{J}=7.5 \mathrm{~Hz}, 3 \mathrm{H}, \mathrm{H}_{6}\right) ; 1.32(\mathrm{dq}, \mathrm{J}=7.5,14.4 \mathrm{~Hz}$, $1 \mathrm{H}, \mathrm{H}_{5}$ or $\left.\mathrm{H}_{11}\right) ; 1.38\left(\mathrm{dq}, \mathrm{J}=7.5,14.4 \mathrm{~Hz}, 1 \mathrm{H}, \mathrm{H}_{5}\right.$ or $\left.\mathrm{H}_{11}\right) ; 1.60\left(\mathrm{dq}, \mathrm{J}=7.5,14.4 \mathrm{~Hz}, 1 \mathrm{H}, \mathrm{H}_{5}\right.$ or $\left.\mathrm{H}_{11}\right) ; 1.76\left(\mathrm{~d}, \mathrm{~J}=13.2 \mathrm{~Hz}, 1 \mathrm{H}, \mathrm{H}_{7}\right) ; 1.99\left(\mathrm{~d}, \mathrm{~J}=13.2 \mathrm{~Hz}, 1 \mathrm{H}, \mathrm{H}_{7}\right) ; 2.32(\mathrm{dq}, \mathrm{J}=7.5,14.4 \mathrm{~Hz}, 1 \mathrm{H}$, $\mathrm{H}_{5}$ or $\left.\mathrm{H}_{11}\right) ; 2.69\left(\mathrm{~d}, \mathrm{~J}=1 \mathrm{~Hz}, 3 \mathrm{H}, \mathrm{H}_{1}\right) ; 5.25\left(\mathrm{dd}, \mathrm{J}=1.5,17.5 \mathrm{~Hz}, 1 \mathrm{H}, \mathrm{H}_{10}\right) ; 5.29(\mathrm{dd}, \mathrm{J}=1.5,11.0$ $\left.\mathrm{Hz}, 1 \mathrm{H}, \mathrm{H}_{10}\right) ; 5.75$ (s, 1H, H $\left.{ }_{14}\right) ; 5.83\left(\mathrm{dd}, \mathrm{J}=11.0,17.5 \mathrm{~Hz}, 1 \mathrm{H}, \mathrm{H}_{9}\right) ; 5.97$ (q, J = 1.0 Hz, 1H, $\left.\mathrm{H}_{3}\right)$; 7.28-7.40 (m, 3H, $\left.\mathrm{H}_{16}, \mathrm{H}_{17}\right)$; 7.48-7.52 (m, 2H, $\left.\mathrm{H}_{15}\right)$; ESMS m/z: $435\left(\mathrm{M}+\mathrm{Na}^{+}\right), 413$ $\left(\mathrm{M}+\mathrm{H}^{+}\right) ; \mathrm{IR}\left(\mathrm{NaCl}\right.$, thin film) $\tilde{\mathrm{v}}_{\max }\left(\mathrm{cm}^{-1}\right) ; 921,1066,1122,1458,1645,2972$. 


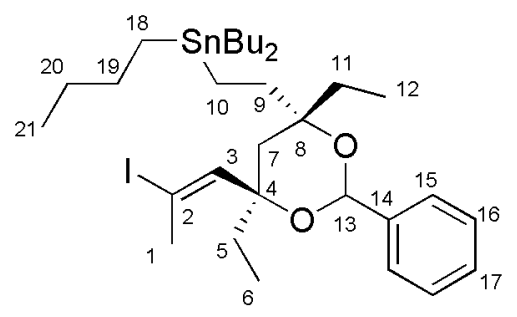

S-12

Characterization of $(4 S, 6 S)$-Tributyl\{2-[4,6-diethyl-6-(2-iodopropenyl)-2-phenyl-1,3dioxan-4-yl]ethyl\}stannane S-12: $\mathrm{R}_{\mathrm{f}} 0.78$ (hexanes/ether 19:1); ${ }^{1} \mathrm{H}$ NMR $\left(500 \mathrm{MHz}, \mathrm{C}_{6} \mathrm{D}_{6}\right) \delta$ 0.89 (t, J = 7.5 Hz, 3H, H $\left.{ }_{12}\right) ; 0.90$ (t, J = 7.6 Hz, 3H, H6); 0.92-0.96 (m, 15H, H $\left.\mathrm{H}_{18}, \mathrm{H}_{21}\right)$; 1.26-1.42 $\left(\mathrm{m}, 8 \mathrm{H}, \mathrm{H}_{5}, \mathrm{H}_{11}, \mathrm{H}_{20}\right) ; 1.52-1.62\left(\mathrm{~m}, 8 \mathrm{H}, \mathrm{H}_{5}, \mathrm{H}_{11}, \mathrm{H}_{19}\right) ; 1.58\left(\mathrm{~d}, \mathrm{~J}=13.2 \mathrm{~Hz}, 1 \mathrm{H}, \mathrm{H}_{7}\right) ; 1.65(\mathrm{~d}, \mathrm{~J}=$ 13.2 Hz, 1H, $\left.\mathrm{H}_{7}\right) ; 1.74-2.02\left(\mathrm{~m}, 4 \mathrm{H}, \mathrm{H}_{9}, \mathrm{H}_{10}\right) ; 2.62\left(\mathrm{q}, \mathrm{J}=1.0 \mathrm{~Hz}, 3 \mathrm{H}, \mathrm{H}_{1}\right) ; 5.88\left(\mathrm{~s}, 1 \mathrm{H}, \mathrm{H}_{13}\right)$; 6.12 (q, J = $\left.1.0 \mathrm{~Hz}, 1 \mathrm{H}, \mathrm{H}_{3}\right) ; 7.09-7.24\left(\mathrm{~m}, 3 \mathrm{H}, \mathrm{H}_{16}, \mathrm{H}_{17}\right) ; 7.62-7.64\left(\mathrm{~m}, 2 \mathrm{H}, \mathrm{H}_{15}\right) ;{ }^{13} \mathrm{C}$ NMR $(100$ $\left.\mathrm{MHz}, \mathrm{CDCl}_{3}\right) \delta 7.9\left(\mathrm{C}_{12}\right), 83.3\left(\mathrm{C}_{6}\right) ; 8.9\left(\mathrm{C}_{18}\right) ; 13.9\left(\mathrm{C}_{21}\right) ; 27.6\left(\mathrm{C}_{20}\right) ; 29.5\left(\mathrm{C}_{19}\right) ; 30.8\left(\mathrm{C}_{9}\right) ; 31.8$ $\left(\mathrm{C}_{10}\right)$; $32.6\left(\mathrm{C}_{1}\right) ; 33.3\left(\mathrm{C}_{5}\right) ; 39.0\left(\mathrm{C}_{11}\right) ; 78.0\left(\mathrm{C}_{8}\right)$; $80.2\left(\mathrm{C}_{4}\right) ; 90.7\left(\mathrm{C}_{2}\right) ; 97.8\left(\mathrm{C}_{13}\right) ; 126.5\left(\mathrm{C}_{15}\right)$; $128.4\left(\mathrm{C}_{16}\right) ; 128.6\left(\mathrm{C}_{17}\right) ; 139.9\left(\mathrm{C}_{14}\right) ; 146.2\left(\mathrm{C}_{3}\right)$; ESMS m/z: $727\left(\mathrm{M}+\mathrm{Na}^{+}\right)$; EIMS m/z: $647(\mathrm{M}-$ $\left.\mathrm{C}_{4} \mathrm{H}_{9}{ }^{+}, 1\right), 361$, (22), 305 (33), 269 (74), 213 (55), 177 (68), 105 (100), 77 (82). 


\section{Synthesis of EpiPlakortone B}

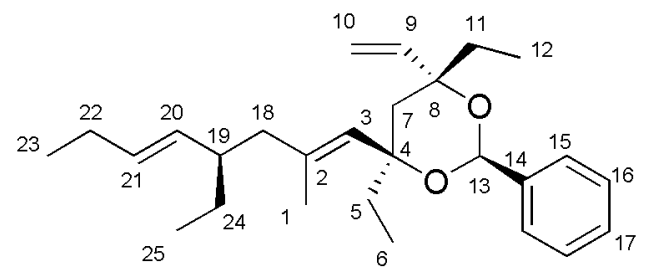

30

Preparation of $(4 S, 4$ ' $R, 6 S)-4,6-D i e t h y l-4-(4-e t h y l-2-m e t h y l o c t a-1,5-d i e n y l)-2-p h e n y l-6-$ vinyl-1,3-dioxane 30: 29 was converted to 30 on a $0.1 \mathrm{mmol}$ scale using the procedure previously reported for the synthesis of 22, yielding 30 (81\%) as a colorless oil after column chromatography $\left(\mathrm{SiO}_{2}\right.$; hexanes/ether 500:1), $\mathrm{R}_{\mathrm{f}} 0.8$ (hexanes/ether 19:1); ${ }^{1} \mathrm{H} \mathrm{NMR}(500 \mathrm{MHz}$, $\left.\mathrm{C}_{6} \mathrm{D}_{6}\right) \delta 0.91\left(\mathrm{t}, \mathrm{J}=7.5 \mathrm{~Hz}, 3 \mathrm{H}, \mathrm{H}_{23}\right.$ or $\left.\mathrm{H}_{25}\right) ; 0.97\left(\mathrm{t}, \mathrm{J}=7.5 \mathrm{~Hz}, 3 \mathrm{H}, \mathrm{H}_{23}\right.$ or $\left.\mathrm{H}_{25}\right) ; 0.99(\mathrm{t}, \mathrm{J}=7.5$ $\mathrm{Hz}, 3 \mathrm{H}, \mathrm{H}_{6}$ or $\left.\mathrm{H}_{12}\right) ; 1.02\left(\mathrm{t}, \mathrm{J}=7.5 \mathrm{~Hz}, 3 \mathrm{H}, \mathrm{H}_{6}\right.$ or $\left.\mathrm{H}_{12}\right) ; 1.18-1.24\left(\mathrm{~m}, 1 \mathrm{H}, \mathrm{H}_{24}\right) ; 1.34(\mathrm{dq}, \mathrm{J}=7.5$, $13.0 \mathrm{~Hz}, 1 \mathrm{H}, \mathrm{H}_{5}$ or $\left.\mathrm{H}_{11}\right) ; 1.40-1.48\left(\mathrm{~m}, 1 \mathrm{H}, \mathrm{H}_{24}\right) ; 1.55$ (dq, J = 7.5, $13.0 \mathrm{~Hz}, 1 \mathrm{H}, \mathrm{H}_{5}$ or $\left.\mathrm{H}_{11}\right) ; 1.63$ $\left(\mathrm{d}, \mathrm{J}=14.0 \mathrm{~Hz}, \mathrm{H}_{7}\right) ; 1.68\left(\mathrm{dq}, \mathrm{J}=7.5,13.0 \mathrm{~Hz}, 1 \mathrm{H}, \mathrm{H}_{5}\right.$ or $\left.\mathrm{H}_{11}\right) ; 1.92\left(\mathrm{~d}, \mathrm{~J}=1.5 \mathrm{~Hz}, 3 \mathrm{H}, \mathrm{H}_{1}\right)$; 1.94-2.10 (m, 5H, H $\left.\mathrm{H}_{18}, \mathrm{H}_{19}, \mathrm{H}_{22}\right) ; 2.06$ (d, J = $\left.14.0 \mathrm{~Hz}, \mathrm{H}_{7}\right) ; 2.44$ (dq, J = 7.5, 13.0 Hz, 1H, H $\left.\mathrm{H}_{11}\right) ; 5.11\left(\mathrm{dd}, 1.5,10.5 \mathrm{~Hz}, 1 \mathrm{H}, \mathrm{H}_{10}\right) ; 5.14\left(\mathrm{ddt}, \mathrm{J}=1.5,8.5,15.0 \mathrm{~Hz}, 1 \mathrm{H}, \mathrm{H}_{20}\right) ; 5.21(\mathrm{q}, \mathrm{J}=1.5$ $\left.\mathrm{Hz}, 1 \mathrm{H}, \mathrm{H}_{3}\right) ; 5.26\left(\mathrm{dd}, \mathrm{J}=1.5,17.5 \mathrm{~Hz}, 1 \mathrm{H}, \mathrm{H}_{10}\right) ; 5.39\left(\mathrm{dt}, \mathrm{J}=6.0,15.0 \mathrm{~Hz}, 1 \mathrm{H}, \mathrm{H}_{21}\right) ; 5.66(\mathrm{dd}, \mathrm{J}$ $\left.=10.5,17.5 \mathrm{~Hz}, 1 \mathrm{H}, \mathrm{H}_{9}\right) ; 5.95\left(\mathrm{~s}, 1 \mathrm{H}, \mathrm{H}_{13}\right) ; 7.12-7.26\left(\mathrm{~m}, 3 \mathrm{H}_{16} \mathrm{H}_{16}, \mathrm{H}_{17}\right) ; 7.76-7.81\left(\mathrm{~m}, 2 \mathrm{H}, \mathrm{H}_{15}\right)$; ${ }^{13} \mathrm{C}$ NMR $\left(125 \mathrm{MHz}, \mathrm{C}_{6} \mathrm{D}_{6}\right) \delta 7.5\left(\mathrm{C}_{12}\right) ; 8.8\left(\mathrm{C}_{6}\right) ; 12.3\left(\mathrm{C}_{25}\right) ; 14.7\left(\mathrm{C}_{23}\right) ; 18.3\left(\mathrm{C}_{1}\right) ; 26.4\left(\mathrm{C}_{22}\right)$; $28.5\left(\mathrm{C}_{24}\right) ; 29.1\left(\mathrm{C}_{11}\right) ; 38.4\left(\mathrm{C}_{5}\right) ; 43.0\left(\mathrm{C}_{19}\right) ; 43.6\left(\mathrm{C}_{18}\right) ; 48.3\left(\mathrm{C}_{7}\right) ; 77.1\left(\mathrm{C}_{4}\right) ; 77.9\left(\mathrm{C}_{8}\right) ; 90.7$ $\left(\mathrm{C}_{13}\right) ; 114.8\left(\mathrm{C}_{10}\right) ; 127.2\left(\mathrm{C}_{3}\right) ; 127.9\left(\mathrm{C}_{17}\right) ; 128.8\left(\mathrm{C}_{16}\right) ; 132.4\left(\mathrm{C}_{15}\right) ; 133.0\left(\mathrm{C}_{21}\right) ; 133.8\left(\mathrm{C}_{20}\right)$;

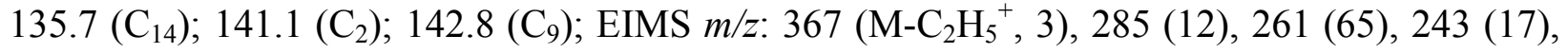
179 (82), 151 (73), 121 (62), 105 (93), 83 (100); ESMS m/z: 419 (M+Na ${ }^{+}$); IR (NaCl, thin film) $\tilde{v}_{\max }\left(\mathrm{cm}^{-1}\right) ; 801,1016,1260,1457,2927$. 


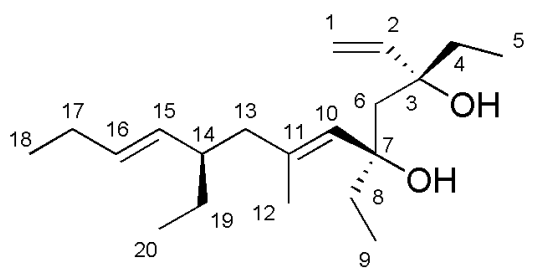

S-14

Preparation of $(3 R, 5 S, 9 S)-3,5,9-T r i e t h y l-7-m e t h y l t r i d e c a-1,6,10-t r i e n e-3,5-d i o l ~ S-14: ~ 30$ was converted to S-14 on a $0.1 \mathrm{mmol}$ scale using the procedure previously reported for the synthesis of 23, yielding S-14 (75\%) as a colorless oil after column chromatography $\left(\mathrm{SiO}_{2}\right.$; hexanes/ether 9:1), $\mathrm{R}_{\mathrm{f}} 0.25$ (hexanes/ether 4:1); ${ }^{1} \mathrm{H} \mathrm{NMR}\left(500 \mathrm{MHz}, \mathrm{C}_{6} \mathrm{D}_{6}\right) \delta 0.91\left(\mathrm{t}, \mathrm{J}=7.5 \mathrm{~Hz}, 3 \mathrm{H}, \mathrm{H}_{18}\right.$ or $\left.\mathrm{H}_{20}\right) ; 0.92\left(\mathrm{t}, \mathrm{J}=7.5 \mathrm{~Hz}, 3 \mathrm{H}, \mathrm{H}_{18}\right.$ or $\left.\mathrm{H}_{20}\right) ; 0.94\left(\mathrm{t}, \mathrm{J}=7.5 \mathrm{~Hz}, 3 \mathrm{H}, \mathrm{H}_{5}\right.$ or $\left.\mathrm{H}_{9}\right) ; 1.00$ (t, J = 7.5 Hz, $3 \mathrm{H}, \mathrm{H}_{5}$ or $\left.\mathrm{H}_{9}\right) ; 1.20-1.30\left(\mathrm{~m}, 2 \mathrm{H}, \mathrm{H}_{19}\right) ; 1.41\left(\mathrm{dq}, \mathrm{J}=7.5,13.0 \mathrm{~Hz}, 1 \mathrm{H}, \mathrm{H}_{4} \mathrm{Or} \mathrm{H}_{8}\right) ; 1.52(\mathrm{dq}, \mathrm{J}=$ 7.5, $13.0 \mathrm{~Hz}, 1 \mathrm{H}, \mathrm{H}_{4}$ or $\left.\mathrm{H}_{8}\right) ; 1.63\left(\mathrm{dq}, \mathrm{J}=7.5,13.0 \mathrm{~Hz}, 1 \mathrm{H}, \mathrm{H}_{4}\right.$ or $\left.\mathrm{H}_{8}\right) ; 1.72$ (d, J = 14.0 Hz, $\mathrm{H}_{6}$ ); $1.74\left(\mathrm{dq}, \mathrm{J}=7.5,13.0 \mathrm{~Hz}, 1 \mathrm{H}, \mathrm{H}_{4}\right.$ or $\left.\mathrm{H}_{8}\right) ; 1.86\left(\mathrm{~d}, \mathrm{~J}=1.5 \mathrm{~Hz}, 3 \mathrm{H}, \mathrm{H}_{12}\right) ; 1.90$ (d, J = 14.0 Hz, $\left.\mathrm{H}_{6}\right)$; 1.98-2.07 (m, 5H, H $\left.\mathrm{H}_{13}, \mathrm{H}_{14}, \mathrm{H}_{17}\right) ; 2.46$ (br s, OH); 3.08 (br s, OH); 5.03 (dd, 2.0, $11.0 \mathrm{~Hz}, 1 \mathrm{H}$, $\left.\mathrm{H}_{1}\right) ; 5.13$ (ddt, $\left.\mathrm{J}=1.5,8.5,15.0 \mathrm{~Hz}, 1 \mathrm{H}, \mathrm{H}_{15}\right) ; 5.14$ (q, J = 1.5 Hz, 1H, $\left.\mathrm{H}_{10}\right) ; 5.39$ (dd, J = 2, 17.5 $\left.\mathrm{Hz}, 1 \mathrm{H}, \mathrm{H}_{1}\right) ; 5.40\left(\mathrm{dt}, \mathrm{J}=6.0,15.0 \mathrm{~Hz}, 1 \mathrm{H}, \mathrm{H}_{16}\right) ; 5.80\left(\mathrm{dd}, \mathrm{J}=11.0,17.5 \mathrm{~Hz}, 1 \mathrm{H}, \mathrm{H}_{2}\right) ;{ }^{13} \mathrm{C}$ NMR $\left(125 \mathrm{MHz}, \mathrm{C}_{6} \mathrm{D}_{6}\right) \delta 8.2\left(\mathrm{C}_{5}\right) ; 8.6\left(\mathrm{C}_{9}\right) ; 12.3\left(\mathrm{C}_{20}\right) ; 17.5\left(\mathrm{C}_{18}\right) ; 26.4\left(\mathrm{C}_{12}\right) ; 28.7\left(\mathrm{C}_{17}\right) ; 32.3\left(\mathrm{C}_{19}\right)$; $36.1\left(\mathrm{C}_{4}\right) ; 36.6\left(\mathrm{C}_{8}\right) ; 43.4\left(\mathrm{C}_{14}\right) ; 48.5\left(\mathrm{C}_{13}\right) ; 50.8\left(\mathrm{C}_{6}\right) ; 76.6\left(\mathrm{C}_{7}\right) ; 77.9\left(\mathrm{C}_{3}\right) ; 112.7\left(\mathrm{C}_{1}\right) ; 128.8$

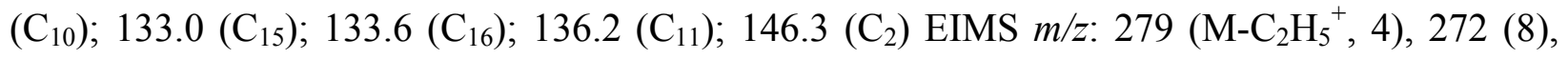
243 (18), 179 (55), 161 (41), 133 (68), 119 (82), 97 (100), 81 (72), 69 (96); EIHRMS m/z: calcd for $\mathrm{C}_{18} \mathrm{H}_{31} \mathrm{O}_{2}\left(\mathrm{M}-\mathrm{C}_{2} \mathrm{H}_{5}{ }^{+}\right), 279.23241$; found 279.23213; ESMS $m / z: 331\left(\mathrm{M}+\mathrm{Na}^{+}\right)$. 


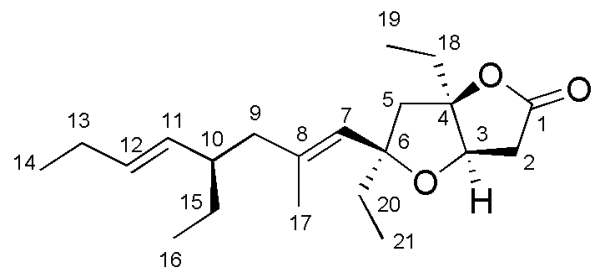

32

Preparation of 3,4-Epiplakortone B 32: S-14 was converted to 32 on a 0.03 mmol scale using the procedure previously reported for the synthesis of $\mathbf{1}$, yielding $32(60 \%)$ as a colorless oil after column chromatography $\left(\mathrm{SiO}_{2}\right.$; hexanes/ether 4:1), $\mathrm{R}_{\mathrm{f}} 0.2$ (hexanes/ether 4:1); ${ }^{1} \mathrm{H}$ NMR $(500$ $\left.\mathrm{MHz}, \mathrm{CDCl}_{3}\right) \delta 0.82\left(\mathrm{t}, \mathrm{J}=7.5 \mathrm{~Hz}, 3 \mathrm{H}, \mathrm{H}_{21}\right) ; 0.87\left(\mathrm{t}, \mathrm{J}=7.5 \mathrm{~Hz}, 3 \mathrm{H}, \mathrm{H}_{14}\right) ; 0.95(\mathrm{t}, \mathrm{J}=7.5 \mathrm{~Hz}$, $\left.\mathrm{H}_{16}\right) ; 1.02\left(\mathrm{t}, \mathrm{J}=7.5 \mathrm{~Hz}, 3 \mathrm{H}, \mathrm{H}_{19}\right) ; 1.30-1.38\left(\mathrm{~m}, 2 \mathrm{H}, \mathrm{H}_{15}\right) ; 1.60$ (dq, J = 7.5, $13.0 \mathrm{~Hz}, 1 \mathrm{H}, \mathrm{H}_{18}$ or $\left.\mathrm{H}_{20}\right) ; 1.69\left(\mathrm{~d}, \mathrm{~J}=1.0 \mathrm{~Hz}, 3 \mathrm{H}, \mathrm{H}_{17}\right) ; 1.75\left(\mathrm{dq}, \mathrm{J}=7.5,13.0 \mathrm{~Hz}, 1 \mathrm{H}, \mathrm{H}_{18}\right.$ or $\left.\mathrm{H}_{20}\right) ; 1.76$ (dq, J = 7.5, $13.0 \mathrm{~Hz}, 1 \mathrm{H}, \mathrm{H}_{18}$ or $\left.\mathrm{H}_{20}\right) ; 1.84\left(\mathrm{dq}, \mathrm{J}=7.5,13.0 \mathrm{~Hz}, 1 \mathrm{H}, \mathrm{H}_{18}\right.$ or $\left.\mathrm{H}_{20}\right) ; 1.88-1.92\left(\mathrm{~m}, 2 \mathrm{H}, \mathrm{H}_{9}\right) ; 1.95$ $\left(\mathrm{d}, \mathrm{J}=13.5 \mathrm{~Hz}, 1 \mathrm{H}, \mathrm{H}_{5}\right) ; 1.96-2.04\left(\mathrm{~m}, 3 \mathrm{H}, \mathrm{H}_{10}, \mathrm{H}_{13}\right) ; 2.46$ (d, J = $\left.13.5 \mathrm{~Hz}, 1 \mathrm{H}, \mathrm{H}_{5}\right) ; 2.65$ (dd, J $\left.=1.5,18.5 \mathrm{~Hz}, 1 \mathrm{H}, \mathrm{H}_{2}\right) ; 2.70\left(\mathrm{dd}, \mathrm{J}=6.0,18.5 \mathrm{~Hz}, 1 \mathrm{H}, \mathrm{H}_{2}\right) ; 4.40\left(\mathrm{dd}, \mathrm{J}=1.5,6.0 \mathrm{~Hz}, 1 \mathrm{H}, \mathrm{H}_{3}\right)$; $5.09\left(\mathrm{ddt}, \mathrm{J}=1.0,8.5,15.5,1 \mathrm{H}, \mathrm{H}_{11}\right) ; 5.25\left(\mathrm{q}, \mathrm{J}=1.0 \mathrm{~Hz}, 1 \mathrm{H}, \mathrm{H}_{7}\right) ; 5.36(\mathrm{dt}, \mathrm{J}=6.5,15.5 \mathrm{~Hz}, 1 \mathrm{H}$, $\left.\mathrm{H}_{12}\right) ;{ }^{13} \mathrm{C} \mathrm{NMR}\left(125 \mathrm{MHz}, \mathrm{CDCl}_{3}\right) \delta 8.8\left(\mathrm{C}_{19}\right) ; 9.1\left(\mathrm{C}_{21}\right) ; 11.8\left(\mathrm{C}_{16}\right) ; 14.3\left(\mathrm{C}_{14}\right) ; 17.9\left(\mathrm{C}_{17}\right) ; 25.8$ $\left(\mathrm{C}_{18}\right) ; 27.7\left(\mathrm{C}_{13}\right) ; 30.8\left(\mathrm{C}_{15}\right) ; 33.8\left(\mathrm{C}_{20}\right) ; 37.7\left(\mathrm{C}_{2}\right) ; 42.9\left(\mathrm{C}_{10}\right) ; 46.9\left(\mathrm{C}_{9}\right) ; 48.6\left(\mathrm{C}_{5}\right) ; 80.5\left(\mathrm{C}_{3}\right)$;

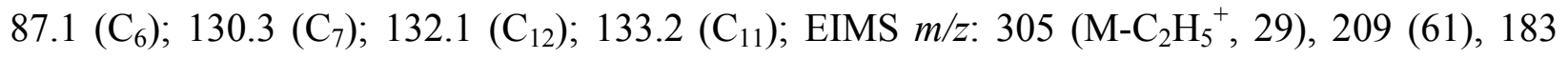
(100), 149 (18), 97 (94), 69 (45); EIHRMS m/z: calcd for $\mathrm{C}_{19} \mathrm{H}_{29} \mathrm{O}_{3}$, 305.21167; found 305.21115 . 


\section{Synthesis of DihydroEpiPlakortone B}

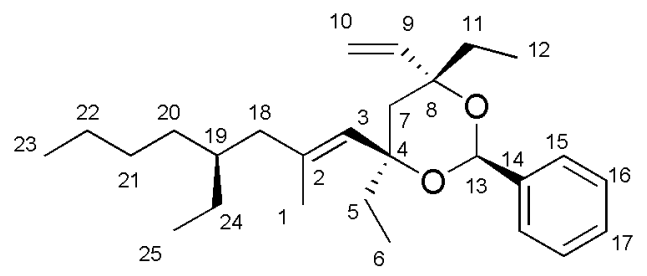

31

Preparation of $\left(4 S, 4^{\prime} R, 6 S\right)-4,6-D i e t h y l-4-(4-e t h y l-2-m e t h y l o c t-1-e n y l)-2-p h e n y l-6-v i n y l-1,3-$

dioxane 31: 29 was converted to 31 on a $0.1 \mathrm{mmol}$ scale using the procedure previously reported for the synthesis of 22, yielding 31 (75\%) as a colorless oil after column chromatography $\left(\mathrm{SiO}_{2}\right.$; hexanes/ether 500:1), $\mathrm{R}_{\mathrm{f}} 0.8$ (hexanes/ether 19:1); ${ }^{1} \mathrm{H}$ NMR (500 MHz, $\left.\mathrm{C}_{6} \mathrm{D}_{6}\right) \delta 0.89(\mathrm{t}, \mathrm{J}=7.5$ $\mathrm{Hz}, 3 \mathrm{H}, \mathrm{H}_{23}$ or $\left.\mathrm{H}_{25}\right) ; 0.91\left(\mathrm{t}, \mathrm{J}=7.5 \mathrm{~Hz}, 3 \mathrm{H}, \mathrm{H}_{23}\right.$ or $\left.\mathrm{H}_{25}\right) ; 0.94\left(\mathrm{t}, \mathrm{J}=7.5 \mathrm{~Hz}, 3 \mathrm{H}, \mathrm{H}_{6}\right.$ or $\left.\mathrm{H}_{12}\right) ; 1.02$ (t, J = 7.5 Hz, $3 \mathrm{H}, \mathrm{H}_{6}$ or $\left.\mathrm{H}_{12}\right)$; 1.22-1.41 (m, $11 \mathrm{H}, \mathrm{H}_{5}$ or $\left.\mathrm{H}_{11}, \mathrm{H}_{18}, \mathrm{H}_{19}, \mathrm{H}_{20}, \mathrm{H}_{21}, \mathrm{H}_{22}, \mathrm{H}_{24}\right) ; 1.53$ $\left(\mathrm{dq}, \mathrm{J}=7.5,13.0 \mathrm{~Hz}, 1 \mathrm{H}, \mathrm{H}_{5}\right.$ or $\left.\mathrm{H}_{11}\right) ; 1.62\left(\mathrm{~d}, \mathrm{~J}=14.0 \mathrm{~Hz}, \mathrm{H}_{7}\right) ; 1.66\left(\mathrm{dq}, \mathrm{J}=7.5,13.0 \mathrm{~Hz}, 1 \mathrm{H}, \mathrm{H}_{5}\right.$ or $\left.\mathrm{H}_{11}\right) ; 1.95\left(\mathrm{~d}, \mathrm{~J}=1.5 \mathrm{~Hz}, 3 \mathrm{H}, \mathrm{H}_{1}\right) ; 1.98\left(\mathrm{dd}, \mathrm{J}=4.0,9.0 \mathrm{~Hz}, 1 \mathrm{H}, \mathrm{H}_{18}\right) ; 2.08$ (d, J = $14.0 \mathrm{~Hz}$, $\left.\mathrm{H}_{7}\right) ; 2.42\left(\mathrm{dq}, \mathrm{J}=7.5,13.0 \mathrm{~Hz}, 1 \mathrm{H}, \mathrm{H}_{5}\right.$ or $\left.\mathrm{H}_{11}\right) ; 5.12\left(\mathrm{dd}, 1.5,11.0 \mathrm{~Hz}, 1 \mathrm{H}, \mathrm{H}_{10}\right) ; 5.24(\mathrm{q}, \mathrm{J}=1.5$ $\left.\mathrm{Hz}, 1 \mathrm{H}, \mathrm{H}_{3}\right) ; 5.26\left(\mathrm{dd}, \mathrm{J}=1.5,17.6 \mathrm{~Hz}, 1 \mathrm{H}, \mathrm{H}_{10}\right) ; 5.66\left(\mathrm{dd}, \mathrm{J}=11.0,17.6 \mathrm{~Hz}, 1 \mathrm{H}, \mathrm{H}_{9}\right) ; 5.95$ (s, $\left.1 \mathrm{H}, \mathrm{H}_{13}\right) ; 7.12-7.26\left(\mathrm{~m}, 3 \mathrm{H}, \mathrm{H}_{16}, \mathrm{H}_{17}\right) ; 7.81\left(\mathrm{dd}, \mathrm{J}=1.0,8.5 \mathrm{~Hz}, \mathrm{H}_{15}\right) ;{ }^{13} \mathrm{C} \mathrm{NMR}(125 \mathrm{MHz}$, $\left.\mathrm{C}_{6} \mathrm{D}_{6}\right) \delta 7.5\left(\mathrm{C}_{6}\right) ; 8.9\left(\mathrm{C}_{12}\right) ; 11.7\left(\mathrm{C}_{25}\right) ; 14.7\left(\mathrm{C}_{23}\right) ; 18.0\left(\mathrm{C}_{1}\right) ; 23.9\left(\mathrm{C}_{22}\right) ; 26.3\left(\mathrm{C}_{24}\right) ; 29.1\left(\mathrm{C}_{21}\right)$; $29.6\left(\mathrm{C}_{5}\right) ; 33.2\left(\mathrm{C}_{11}\right) ; 37.3\left(\mathrm{C}_{20}\right) ; 38.3\left(\mathrm{C}_{19}\right) ; 42.9\left(\mathrm{C}_{18}\right) ; 46.9\left(\mathrm{C}_{7}\right) ; 77.2\left(\mathrm{C}_{4}\right) ; 77.9\left(\mathrm{C}_{8}\right) ; 90.8$ $\left(\mathrm{C}_{13}\right)$; $114.8\left(\mathrm{C}_{10}\right) ; 127.2\left(\mathrm{C}_{17}\right) ; 128.8\left(\mathrm{C}_{16}\right) ; 128.9\left(\mathrm{C}_{15}\right) ; 132.7\left(\mathrm{C}_{3}\right) ; 136.3\left(\mathrm{C}_{14}\right) 141.1\left(\mathrm{C}_{2}\right)$;

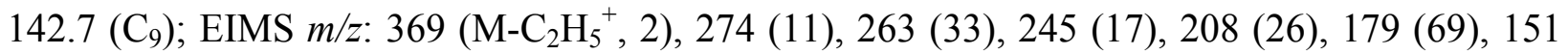
(61), 105 (88), 95 (62), 83 (89), 69 (100); EIHRMS m/z: calcd for $\mathrm{C}_{25} \mathrm{H}_{37} \mathrm{O}_{2}\left(\mathrm{M}-\mathrm{C}_{2} \mathrm{H}_{5}^{+}\right.$), 369.27935; found 369.27780; ESMS m/z: $421\left(\mathrm{M}+\mathrm{Na}^{+}\right)$; IR $\left(\mathrm{NaCl}\right.$, thin film) $\tilde{\mathrm{v}}_{\max }\left(\mathrm{cm}^{-1}\right) ; 801$, 1016, 1260, 1457, 2927. 


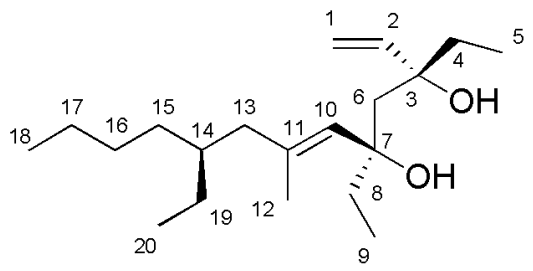

S-15

Preparation of $(3 R, 5 S, 9 S)-3,5,9-T r i e t h y l-7-m e t h y l t r i d e c a-1,6-d i e n e-3,5-d i o l ~ S-15: 31$ was converted to S-15 on a $0.1 \mathrm{mmol}$ scale using the procedure previously reported for the synthesis of 23, yielding S-15 (79\%) as a colorless oil after column chromatography $\left(\mathrm{SiO}_{2}\right.$; hexanes/ether 9:1), $\mathrm{R}_{\mathrm{f}} 0.25$ (hexanes/ether 4:1); ${ }^{1} \mathrm{H}$ NMR $\left(500 \mathrm{MHz}, \mathrm{C}_{6} \mathrm{D}_{6}\right) \delta 0.88\left(\mathrm{t}, \mathrm{J}=7.5 \mathrm{~Hz}, 3 \mathrm{H}, \mathrm{H}_{18}\right.$ or $\left.\mathrm{H}_{20}\right) ; 0.90\left(\mathrm{t}, \mathrm{J}=7.5 \mathrm{~Hz}, 3 \mathrm{H}, \mathrm{H}_{18}\right.$ or $\left.\mathrm{H}_{20}\right) ; 0.93$ (t, J = $7.5 \mathrm{~Hz}, 3 \mathrm{H}, \mathrm{H}_{5}$ or $\mathrm{H}_{9}$ ); 0.94 (t, J = $7.5 \mathrm{~Hz}$, $3 \mathrm{H}, \mathrm{H}_{5}$ or $\left.\mathrm{H}_{9}\right)$; 1.20-1.36 (m, 9H, $\left.\mathrm{H}_{14}, \mathrm{H}_{15}, \mathrm{H}_{16}, \mathrm{H}_{17}, \mathrm{H}_{19}\right)$; 1.41 (dq, J = 7.5, $13.0 \mathrm{~Hz}, 1 \mathrm{H}, \mathrm{H}_{4}$ or $\left.\mathrm{H}_{8}\right) ; 1.54\left(\mathrm{dq}, \mathrm{J}=7.5,13.0 \mathrm{~Hz}, 1 \mathrm{H}, \mathrm{H}_{4}\right.$ or $\left.\mathrm{H}_{8}\right) ; 1.66\left(\mathrm{dq}, \mathrm{J}=7.5,13.0 \mathrm{~Hz}, 1 \mathrm{H}, \mathrm{H}_{4}\right.$ or $\left.\mathrm{H}_{8}\right) ; 1.71$ (d, $\left.\mathrm{J}=14.0 \mathrm{~Hz}, \mathrm{H}_{6}\right) ; 1.74\left(\mathrm{dq}, \mathrm{J}=7.5,13.0 \mathrm{~Hz}, 1 \mathrm{H}, \mathrm{H}_{4}\right.$ or $\left.\mathrm{H}_{8}\right) ; 1.87$ (d, J = 1.0 Hz, 3H, H12); $1.88-$ $1.91\left(\mathrm{~m}, 2 \mathrm{H}, \mathrm{H}_{13}\right) ; 1.91\left(\mathrm{~d}, \mathrm{~J}=14.0 \mathrm{~Hz}, \mathrm{H}_{6}\right) ; 2.62$ (br s, OH); 3.14 (br s, OH); 5.04 (dd, 2.0, 10.5 $\left.\mathrm{Hz}, 1 \mathrm{H}, \mathrm{H}_{1}\right) ; 5.17$ (q, J = 1.0 Hz, 1H, $\left.\mathrm{H}_{10}\right) ; 5.40$ (dd, J = 2.0, $\left.17.0 \mathrm{~Hz}, 1 \mathrm{H}, \mathrm{H}_{1}\right) ; 5.77$ (dd, J = 10.5,

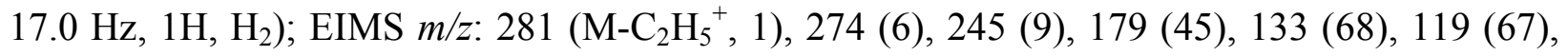
105 (93), 95 (100), 81 (71), 67 (69); ESMS m/z: $333\left(\mathrm{M}+\mathrm{Na}^{+}\right)$.

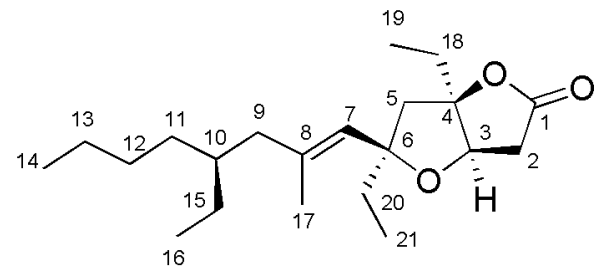

33

Preparation of 3,4-Epi-11,12-dihydroplakortone B 33: S-15 was converted to 33 on a 0.05 mmol scale using the procedure previously reported for the synthesis of $\mathbf{1}$, yielding $33(65 \%)$ as a colorless oil after column chromatography $\left(\mathrm{SiO}_{2}\right.$; hexanes/ether 4:1), $\mathrm{R}_{\mathrm{f}} 0.2$ (hexanes/ether 4:1); ${ }^{1} \mathrm{H}$ NMR $\left(500 \mathrm{MHz}, \mathrm{CDCl}_{3}\right) \delta 0.82\left(\mathrm{t}, \mathrm{J}=7.5 \mathrm{~Hz}, 3 \mathrm{H}, \mathrm{H}_{16}\right) ; 0.889\left(\mathrm{t}, \mathrm{J}=7.5 \mathrm{~Hz}, 3 \mathrm{H}, \mathrm{H}_{14}\right) ; 0.890$ $\left(\mathrm{t}, \mathrm{J}=7.5 \mathrm{~Hz}, \mathrm{H}_{21}\right) ; 1.02$ (t, J = 7.5 Hz, 3H, H $\left.\mathrm{H}_{19}\right) ; 1.16-1.32\left(\mathrm{~m}, 9 \mathrm{H}, \mathrm{H}_{10}, \mathrm{H}_{11}, \mathrm{H}_{12}, \mathrm{H}_{13}, \mathrm{H}_{15}\right) ; 1.60$ 
$\left(\mathrm{dq}, \mathrm{J}=7.5,13.0 \mathrm{~Hz}, 1 \mathrm{H}, \mathrm{H}_{18}\right.$ or $\left.\mathrm{H}_{20}\right) ; 1.72\left(\mathrm{~d}, \mathrm{~J}=1.0 \mathrm{~Hz}, 3 \mathrm{H}, \mathrm{H}_{17}\right) ; 1.75(\mathrm{dq}, \mathrm{J}=7.5,13.0 \mathrm{~Hz}$, $1 \mathrm{H}, \mathrm{H}_{18}$ or $\left.\mathrm{H}_{20}\right) ; 1.76\left(\mathrm{dq}, \mathrm{J}=7.5,13.0 \mathrm{~Hz}, 1 \mathrm{H}, \mathrm{H}_{18}\right.$ or $\left.\mathrm{H}_{20}\right) ; 1.84\left(\mathrm{dq}, \mathrm{J}=7.5,13.0 \mathrm{~Hz}, 1 \mathrm{H}, \mathrm{H}_{18}\right.$ or $\left.\mathrm{H}_{20}\right)$; 1.88-1.92 (m, 2H, H $)$; 1.95 (d, J = 13.5 Hz, 1H, H $\mathrm{H}_{5} ; 2.46$ (d, J = $\left.13.5 \mathrm{~Hz}, 1 \mathrm{H}, \mathrm{H}_{5}\right) ; 2.65$ $\left(\mathrm{dd}, \mathrm{J}=1.5,18.5 \mathrm{~Hz}, 1 \mathrm{H}, \mathrm{H}_{2}\right) ; 2.70\left(\mathrm{dd}, \mathrm{J}=6.0,18.5 \mathrm{~Hz}, 1 \mathrm{H}, \mathrm{H}_{2}\right) ; 4.40$ (dd, J = 1.5, 6.0 Hz, 1H, $\left.\mathrm{H}_{3}\right) ; 5.25\left(\mathrm{q}, \mathrm{J}=1.0 \mathrm{~Hz}, 1 \mathrm{H}, \mathrm{H}_{7}\right) ;{ }^{13} \mathrm{C} \mathrm{NMR}\left(125 \mathrm{MHz}, \mathrm{CDCl}_{3}\right) \delta 8.8\left(\mathrm{C}_{19}\right) ; 9.1\left(\mathrm{C}_{21}\right) ; 10.6\left(\mathrm{C}_{16}\right)$; $14.4\left(\mathrm{C}_{14}\right) ; 17.5\left(\mathrm{C}_{17}\right) ; 23.3\left(\mathrm{C}_{13}\right) ; 25.4\left(\mathrm{C}_{18}\right) ; 29.1\left(\mathrm{C}_{15}\right) ; 30.8\left(\mathrm{C}_{12}\right) ; 32.8\left(\mathrm{C}_{20}\right) ; 33.9\left(\mathrm{C}_{11}\right) ; 36.8$ $\left(\mathrm{C}_{10}\right) ; 37.8\left(\mathrm{C}_{2}\right) ; 45.4\left(\mathrm{C}_{9}\right) ; 48.6\left(\mathrm{C}_{5}\right) ; 80.6\left(\mathrm{C}_{3}\right) ; 87.1\left(\mathrm{C}_{6}\right) ; 97.7\left(\mathrm{C}_{4}\right) ; 130.2\left(\mathrm{C}_{7}\right) ; 138.1\left(\mathrm{C}_{8}\right)$;

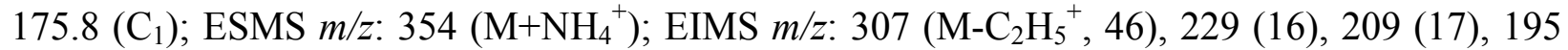
(100), 163 (19), 119 (24), 95 (33), 81 (45); EIHRMS m/z: calcd for $\mathrm{C}_{19} \mathrm{H}_{31} \mathrm{O}_{3}, 307.22732$; found 307.22847.

\section{References}

(1) Kuehne, M. E.; Matson, P. A.; Bornmann, W. G., J. Org. Chem. 1991, 56, 513-528.

(2) Blakemore, P. R.; Cole, W. J.; Kocienski, P. J.; Morley, A., Synlett 1998, 26-28.

(3) Roush, W. R.; Adam, M. A.; Peseckis, S. M., Tetrahedron Letters 1983, 24, 1377-1380.

(4) Patil, A. D.; Freyer, A. J.; Bean, M. F.; Carte, B. K.; Westley, J. W.; Johnson, R. K.; Lahouratate, P. Tetrahedron 1996, 52, 377-394. 